\title{
ASYMPTOTIC ESCAPE RATES AND LIMITING DISTRIBUTIONS FOR MULTIMODAL MAPS
}

\author{
MARK F. DEMERS AND MIKE TODD
}

\begin{abstract}
We consider multimodal maps with holes and study the evolution of the open systems with respect to equilibrium states for both geometric and Hölder potentials. For small holes, we show that a large class of initial distributions share the same escape rate and converge to a unique absolutely continuous conditionally invariant measure; we also prove a variational principle connecting the escape rate to the pressure on the survivor set, with no conditions on the placement of the hole. Finally, introducing a weak condition on the centre of the hole, we prove scaling limits for the escape rate for holes centred at both periodic and nonperiodic points, as the diameter of the hole goes to zero.
\end{abstract}

\section{INTRODUCTION}

Dynamical systems with holes arise naturally in the study of systems whose domain is not invariant under the dynamics. They have been studied in connection with absorbing states in Markov chains [V, FKMP], metastable states in deterministic systems [DoW, BV1, GHW] and neighbourhoods of nonattracting invariant sets $[\mathrm{Y}]$, as well as in components of large systems of interacting components in non-equilibrium statistical mechanics [DGK].

In the present paper, for a class of multimodal maps with holes in the form of intervals, we study the escape rates and limiting behaviours of the open systems with respect to equilibrium states and conformal measures for broad classes of potentials. The systems in question have exponential rates of escape 1 in which the escape rate and limiting behaviour of the open system is expressed through the existence and properties of a physical conditionally invariant measure, absolutely continuous with respect to a given conformal measure. In this setting, given a map $f: I \circlearrowleft$ and identifying a set $H \subset I$ as a hole, one defines the open system by $\stackrel{\circ}{f}=\left.f\right|_{I^{1}}$, where $I^{1}=(I \backslash H) \cap f^{-1}(I \backslash H)$. A conditionally invariant measure $\mu$ is a Borel probability measure satisfying,

$$
\mu(A)=\frac{\stackrel{\circ}{*}_{*} \mu(A)}{\dot{\circ}_{*} \mu(I)} \quad \text { for all Borel } A \subset I .
$$

The evolution of measures in the open system is described by the sequence $f_{*}^{n} \mu_{0} / f_{*}^{n} \mu_{0}(I)$ for initial distributions $\mu_{0}$. If the limit of such a sequence exists and is independent of $\mu_{0}$ for a reasonable class of initial distributions, we call the resulting measure a limiting (or physical) conditionally invariant measure. For open systems with exponential rates of escape, the typical agenda of strong dynamical properties includes a common rate of escape for natural classes of densities, the convergence of such densities to a limiting conditionally invariant measure under iteration of the dynamics, and a

Date: January 19, 2020.

Part of this work was completed at CIRM, Luminy in 2017 and 2018, at ICMS, Scotland in 2018, and during visits of MT to Fairfield University in 2017, 2018 and 2019. MD is partially supported by NSF grant DMS 1800321.

${ }^{1}$ For systems with subexponential rates of escape, the results are qualitatively different since there can be no conditionally invariant limiting distribution $[\mathrm{DF}$. See [DG, FMS, $\mathrm{APT}, \mathrm{DR}, \mathrm{DT}$, $\mathrm{BDT}$ ] for examples of studies in the subexponential regime. 
variational principle connecting the escape rate to the pressure of the open system on the survivor set, the (singular) set of points which never enters the hole.

Such results have been obtained primarily for uniformly hyperbolic systems, beginning with expanding maps [PY, CMS, [LM], Anosov diffeomorphisms [CM, CMT], finite [FP] and countable [DIMMY] state topological Markov chains, and dispersing billiards [DWY, D2]. Their extension to nonuniformly hyperbolic systems has been primarily restricted to uni- and multi-modal interval maps [BDM, DT1, PU] and intermittent maps [DT2].

The purpose of the present paper is to prove strong hyperbolic properties for open systems associated with multimodal maps in greater generality and removing many of the technical assumptions made in previous works. As such, the present paper represents a significant simplification and extension of results available in the context of nonuniformly hyperbolic open systems. Previous works in the setting of unimodal maps with holes have required strong conditions both on the map (Misiurewicz maps in [D1]; a Benedicks-Carleson condition in [BDM, DT1]; a topologically tame condition in [PU]), and on the placement of the hole (slow approach to (see [BDM], DT1]), or complete avoidance of (see [PU]), the post critical set by the boundary or centre of the hole).

The principal innovation we introduce to the study of open systems in this paper is the use of Hofbauer extensions, a type of Markov extension of the original system. Introduced in $[\mathrm{H}]$, they have been used extensively in the study of interval maps. However, to date, they have not been implemented for systems with holes. In this paper we construct Hofbauer extensions of our open system, with additional cuts added to our partition depending on the boundary and centre of our hole. Doing so enables us to consider the lift of the hole as a union of 1-cylinders in the extension. Leveraging recent estimates on complexity from [DoT], we proceed to build an induced map and related Young tower over the Hofbauer extension in order to apply the framework developed in DT2 for Young towers with holes.

This two-step approach (rather than simply constructing a Young tower for the open system directly) allows us to remove many of the technical assumptions needed in previous works for interval maps with holes, as described above. Indeed, we establish the standard suite of strong hyperbolic properties for the open system assuming only that the hole is a finite union of small intervals (Theorem 3.1), entirely eliminating the need for previous assumptions on its placement or on the orbits of its boundary points. We also prove the scaling limits for the escape rate as the hole shrinks to a point under much weaker assumptions than used previously (Theorems 3.5 and 3.7). In addition, we greatly broaden the class of potentials we are able to treat in this setting: we treat all Hölder continuous potentials, as well as the geometric potentials $\phi=-t \log |D f|$ for an interval of $t$ containing [0,1); if the map satisfies a Collet-Eckmann condition, we treat $t \geqslant 1$ as well. This is in contrast to [DT1] which restricted $t$ to a small interval around 1, and [PU] which treated only Hölder potentials with bounded variation.

The paper is organised as follows. In Section 2 we define the class of maps and potentials we shall study, and recall important definitions regarding pressure and open systems. In Section 3 we state our main results, and in Section 4 we carry out our main construction of the Hofbauer extensions and associated induced maps, proving that they enjoy tail bounds and mixing properties that are uniform in the size of the hole. In Section 5 we prove the key spectral properties for the induced open system, which are then leveraged in Section 6 for Young towers, and in Section 7 to establish the small hole asymptotic. 


\section{SETup}

2.1. Dynamics. For $I$ denoting the unit interval, let $\mathcal{F}$ denote the class of $C^{3}$ maps $f: I \rightarrow I$ with

- all critical points non-flat: there exists a finite set Crit $\subset I$ such that for each $c \in$ Crit there is a $C^{3}$ diffeomorphism $\phi$ in a neighbourhood of $c$ with $\phi(c)=0$ such that $f(x)=$ $\pm|\phi(x)|^{d}+f(c)$ for some $d>1$, the order of $c$;

- negative Schwarzian derivative, i.e., $\frac{D^{3} f}{D f}-\frac{3}{2}\left(\frac{D^{2} f}{D f}\right)^{2} \leqslant 0$;

- the locally eventually onto (leo)/topologically exact condition: for any open set $U \subset I$ there exists $n \in \mathbb{N}$ such that $f^{n}(U)=I$, a form of topological transitivity;

- for each $c$,

$$
\left|D f^{n}(f(c))\right| \rightarrow \infty
$$

Note that it is possible to weaken the conditions listed here, but this would lead to a significantly more complex exposition.

Sometimes we will require a stronger condition: we say that $f$ satisfies the Collet-Eckmann condition if there exist $C, \gamma>0$ such that for each $c \in$ Crit, and all $n \in \mathbb{N}$,

$$
\left|D f^{n}(f(c))\right| \geqslant C e^{\gamma n} \text {. }
$$

2.2. Potentials, pressure and equilibrium states. Given $f \in \mathcal{F}$ we let $\mathcal{M}$ denote the set of $f$-invariant probability measures. Then for a potential $\phi: \rightarrow[-\infty, \infty]$, we define the pressure by

$$
P(\phi):=\sup \left\{h_{\mu}(f)+\int \phi d \mu: \mu \in \mathcal{M} \text { and } \mu(-\phi)<\infty\right\} .
$$

A measure $\mu \in \mathcal{M}$ is called an equilibrium state for $\phi$ if $h_{\mu}(f)+\int \phi d \mu=P(\phi)$.

Given $\phi: I \rightarrow[-\infty, \infty]$, we say that a sigma-finite measure $m_{\phi}$ is $\phi$-conformal if whenever $U$ is a Borel set and $f: U \rightarrow f(U)$ is a bijection then

$$
m_{\phi}(f(U))=\int_{U} e^{-\phi} d m_{\phi}
$$

(For example, Lebesgue measure is - $\log |D f|$-conformal.) Notice that we can iterate this relation: if $f^{n}: U \rightarrow f(U)$ is a bijection, then

$$
m_{\phi}\left(f^{n}(U)\right)=\int_{U} e^{-S_{n} \phi} d m_{\phi},
$$

where $S_{n} \phi=\sum_{i=0}^{n-1} \phi \circ f^{i}$. We will also be interested in functions $\psi: I \rightarrow[-\infty, \infty]$ cohomologous to $\phi$; namely, there exists a function $h$ such that $\phi=\psi+h-h \circ f$. These functions share equilibrium states, though they may produce different, but equivalent, conformal measures.

We will consider equilibrium states for two types of potentials: Hölder continuous potentials and geometric potentials.

(i) Hölder continuous potentials. In [LR-L it was shown that any Hölder potential $\phi$ is cohomologous to a Hölder potential $\tilde{\phi}$ with $\tilde{\phi}<P(\tilde{\phi})$ on $I$ (note that there can be many such potentials). It is therefore no loss of generality to assume, as we will throughout, that for our Hölder potentials, $\phi<P(\phi)$. 
(ii) Geometric potentials. We set $\phi=-\log |D f|$ and consider the family $\{t \phi\}_{t \in \mathbb{R}}$. We let $p_{t}:=$ $P(t \phi)$ and denote $m_{t}=m_{t \phi-p_{t}}$ if this measure exists. For a $p$-periodic point $x$, define its Lyapunov exponent by $\lambda(x):=\frac{1}{p} \log \left|D f^{p}(x)\right|$. As in [PR-L, Appendix A], for $f \in \mathcal{F}$ and $x \in I$, it is always the case that $\lambda(x)>0$. Then define

$$
\lambda_{\min }=\inf \{\lambda(x): x \text { is periodic }\} \text { and } \lambda_{\max }:=\sup \{\lambda(x): x \text { is periodic }\}
$$

For $\mu \in \mathcal{M}$, let its Lyapunov exponent be defined by $\lambda(\mu):=\int \log |D f| d \mu$. By [PR-L, Proposition 4.7], if $f \in \mathcal{F}$ then

$$
\inf \{\lambda(\mu): \mu \in \mathcal{M}\}=\lambda_{\min } \text { and } \sup \{\lambda(\mu): \mu \in \mathcal{M}\}=\lambda_{\max } .
$$

Noting from the definition of pressure that $p_{t} \geqslant-t \lambda_{\min }$, we define

$$
t^{+}:=\sup \left\{t \in \mathbb{R}: p_{t}>-t \lambda_{\min }\right\} \text { and } t^{-}:=\inf \left\{t \in \mathbb{R}: p_{t}>-t \lambda_{\max }\right\} .
$$

These are referred to as the freezing point and the condensation point of $f$, respectively. It is immediate that $t^{-}<0$. For $f \in \mathcal{F}$, there is always an acip, which implies that $p_{1}=0$ and $t^{+} \geqslant 1$. As in [PR-L], (CE) implies $t^{+}>1$.

Definition 2.1. We shall call a potential $\phi$ admissible if either: (a) $\phi$ is Hölder continuous and $\phi<P(\phi)$ on $I$; or $(b) \phi=-t \log |D f|$ with $t \in\left(t^{-}, t^{+}\right)$.

For each admissible $\phi$,

$$
P(\phi)=\sup \left\{h_{\mu}(f)+\int \phi d \mu: \mu \in \mathcal{M}, \mu(-\phi)<\infty \text { and } h_{\mu}(f)>0\right\},
$$

and there is a unique equilibrium state which is exponentially mixing: for geometric potentials with $t \in\left(t^{-}, t^{+}\right)$, this follows for example by [IT1, Theorem A]; in the Hölder case this follows from LR-L, Theorem A]. Moreover, each equilibrium state is absolutely continuous with respect to a unique conformal measure, which is shown to exist in, for example, [IT2, Appendix B]. Throughout, we will denote the normalised potential by $\varphi=\phi-P(\phi)$, and say that $\varphi$ is admissible whenever $\phi$ is. Moreover, we let $m_{\varphi}$ and $\mu_{\varphi}$ denote the $\varphi$-conformal measure and the equilibrium state, respectively. We may drop the $\varphi$ when the potential is clear.

2.3. Puncturing the system. Choose $z \in I$, and let $H_{\varepsilon}=(z-\varepsilon, z+\varepsilon) \subset I$ be an interval. Denote by $\stackrel{\circ}{I}=I \backslash H_{\varepsilon}$, and in general by $\stackrel{\circ}{I}^{n}=\cap_{i=0}^{n} f^{-i} \stackrel{\circ}{I}$, the set of points that do not enter $H_{\varepsilon}$ in the first $n$ iterates. The sequence of maps $f^{n}:=\left.f^{n}\right|_{I^{n}}$ defines the corresponding open system.

We define the upper and lower escape rates through $H_{\varepsilon}$ by

$$
\log \bar{\lambda}_{\varepsilon}:=\limsup _{n \rightarrow \infty} \frac{1}{n} \log \mu_{\varphi}\left(\stackrel{\circ}{I}^{n}\right) \quad \text { and } \quad \log \underline{\lambda}_{\varepsilon}:=\liminf _{n \rightarrow \infty} \frac{1}{n} \log \mu_{\varphi}\left(\stackrel{\circ}{I}^{n}\right)
$$

When the two quantities coincide, we denote them by $\log \lambda_{\varepsilon}$, and call $-\log \lambda_{\varepsilon}$ the escape rate with respect to $\mu_{\varphi}$.

Given a potential $\phi$, once a hole $H_{\varepsilon}$ is introduced, the punctured potential is defined by $\phi^{H_{\varepsilon}}=\phi$ on $\stackrel{\circ}{I}$ and $\phi^{H_{\varepsilon}}=-\infty$ on $H_{\varepsilon}$. $P\left(\phi^{H_{\varepsilon}}\right)$ denotes the pressure of the punctured potential, and it follows from the requirement $\mu\left(-\phi^{H_{\varepsilon}}\right)<\infty$ that the supremum for this pressure is restricted to $f$-invariant measures that are supported on the survivor set $\stackrel{\circ}{I}^{\infty}:=\cap_{n=0}^{\infty} \stackrel{\circ}{I}^{n}$.

We will be interested in establishing convergence for limits of the form, $f_{*}^{n} \mu / f_{*}^{n} \mu(I)$ for measures $\mu$ which are absolutely continuous with respect to the conformal measure $m_{\varphi}$. To this end, define 
the transfer operator corresponding to the potential $\varphi$ by,

$$
\mathcal{L}_{\varphi} \psi(x)=\sum_{y \in f^{-1} x} \psi(y) e^{\varphi(y)}, \quad \text { for } \psi \in L^{1}\left(m_{\varphi}\right) .
$$

Similarly, the punctured transfer operator for the open system is defined by

$$
{\stackrel{\circ}{\mathcal{L}^{H} H_{\varepsilon}}} \psi(x)=\mathcal{L}_{\varphi}\left(1_{\check{I}^{1}} \psi\right)(x)=\sum_{y \in \dot{f}^{-1} x} \psi(y) e^{\varphi(y)} .
$$

Due to the conformality of $m_{\varphi}$, we have

$$
\int_{I} \dot{\mathcal{L}}_{\varphi^{H_{\varepsilon}}}^{n} \psi d m_{\varphi}=\int_{\dot{I}^{n}} \psi d m_{\varphi}, \quad \text { for all } n \geq 1
$$

which relates the escape rate with respect to the measure $\psi d m_{\varphi}$ to the spectral radius of $\dot{\mathcal{L}}_{\varphi^{H}{ }_{\varepsilon}}$.

\section{Results}

3.1. Small hole, general placement. Theorem 3.1 proves the standard suite of strong hyperbolic properties for the open system. As noted in the introduction, it is a significant improvement over [BDM], [DT1] and [PU] which had similar results under much more restrictive assumptions on the map, the potential and the hole.

Theorem 3.1. Let $f \in \mathcal{F}$ and $\phi$ be an admissible potential, with normalised version $\varphi=\phi-P(\phi)$. Let $z \in I$, and for $\varepsilon>0$, set $H_{\varepsilon}(z)=(z-\varepsilon, z+\varepsilon)$. Suppose that $\varepsilon^{*}>0$ is sufficiently small so that $-\log \bar{\lambda}_{\varepsilon^{*}}<\alpha$, where $\alpha>0$ is the tail decay rate from Theorem 4.10. Then the following hold for all $0<\varepsilon \leqslant \min \left\{\varepsilon_{1}^{*}, \varepsilon^{*}\right\}$, where $\varepsilon_{1}^{*}$ is from Lemma 6.2.

(a) The escape rate $-\log \lambda_{\varepsilon}$ exists, and $\lambda_{\varepsilon}<1$ is the spectral radius of the punctured transfer operator on the associated Young tower. The associated eigenvector projects to a nonnegative function $\stackrel{\circ}{g}_{\varepsilon}$, which is bounded away from zero on $I \backslash H_{\varepsilon}$ and satisfies $\stackrel{\circ}{\mathcal{L}}_{\varphi} H_{\varepsilon} \stackrel{\circ}{g}_{\varepsilon}=\lambda_{\varepsilon} \stackrel{\circ}{g}_{\varepsilon}$.

(b) There is a unique $\left(\phi^{H_{\varepsilon}}-P\left(\phi^{H_{\varepsilon}}\right)\right)$-conformal measure $m_{H_{\varepsilon}}$. This is singular with respect to $m_{\varphi}$ and supported on $\stackrel{\circ}{I}^{\infty}$.

(c) The measure $\nu_{H_{\varepsilon}}:=\stackrel{\circ}{g}_{\varepsilon} m_{H_{\varepsilon}}$ is the unique equilibrium state for $\phi^{H_{\varepsilon}}-P\left(\phi^{H_{\varepsilon}}\right)$; in particular,

$$
\log \lambda_{\varepsilon}=P\left(\phi^{H_{\varepsilon}}\right)-P(\phi)=P\left(\varphi^{H_{\varepsilon}}\right)=h_{\nu_{H_{\varepsilon}}}(f)+\int \varphi^{H_{\varepsilon}} d \nu_{H_{\varepsilon}} .
$$

Moreover, $\nu_{H_{\varepsilon}}$ is supported on $\stackrel{\circ}{I}^{\infty}$ and can be realised as the limit,

$$
\nu_{H_{\varepsilon}}(\psi)=\lim _{n \rightarrow \infty} \lambda_{\varepsilon}^{-n} \int_{I^{n}} \psi \stackrel{\circ}{g}_{\varepsilon} d m_{\varphi}, \quad \text { for all } \psi \in C^{0}(I) .
$$

(d) The measure $\mu_{\varphi}^{H_{\varepsilon}}:=\stackrel{\circ}{g}_{\varepsilon} m_{\varphi}$ is a conditionally invariant measure supported on $I \backslash H_{\varepsilon}$ with eigenvalue $\lambda_{\varepsilon}$ and is a limiting distribution in the following sense. Fix $\varsigma>0$ and let $\psi \in C^{\varsigma}(I)$ satisfy $\psi \geqslant 0$, with $\nu_{H_{\varepsilon}}(\psi)>0$. Then

$$
\left|\frac{\stackrel{\circ}{\mathcal{L}}_{\varphi^{H_{\varepsilon}}}^{n} \psi}{\left|\dot{\mathcal{L}}_{\varphi^{H_{\varepsilon}}}^{n} \psi\right|_{L^{1}\left(m_{\varphi}\right)}}-\stackrel{\circ}{g}_{\varepsilon}\right|_{L^{1}\left(m_{\varphi}\right)} \leqslant C \vartheta^{n}|\psi|_{C^{\varsigma}(I)}
$$

for some $C>0$ independent of $\psi$, and $\vartheta<1$ depending only on $\varsigma$.

The techniques also imply that $\nu_{H_{\varepsilon}}, \mu_{\varphi}^{H_{\varepsilon}} \rightarrow \mu_{\phi}$ as $\varepsilon \rightarrow 0$. Note that the techniques of the proof also extend to finite unions of intervals as our hole as the only condition required on the hole in [DT2] is $-\log \bar{\lambda}_{\varepsilon}<\alpha$. We prove this theorem in Section 6.2. 
Remark 3.2. In fact, we prove convergence to the accim for a larger class of initial densities than $C^{\varsigma}(I)$. It only matters that $\psi$ satisfies $\nu_{H_{\varepsilon}}(\psi)>0$ and that it can be realised as the projection of an element in a certain function space on the related Young tower. So for example, any function of the form $\psi=\tilde{\psi} g_{\varphi}$ also satisfies (3.1), where $\tilde{\psi} \in C^{\varsigma}(I)$ and $g_{\varphi}=\frac{d \mu_{\varphi}}{d m_{\varphi}}$.

The following lemma shows that we can always choose $\varepsilon^{*}>0$ small enough so that $-\log \bar{\lambda}_{\varepsilon^{*}}<\alpha$, and hence the theorem applies to all small holes.

Lemma 3.3. Suppose $\phi$ is an admissible potential and $f \in \mathcal{F}$. For any $z \in I$, and hole $H_{\varepsilon}(z)=$ $(z-\varepsilon, z+\varepsilon)$, it holds that $\lim _{\varepsilon \rightarrow 0} \bar{\lambda}_{\varepsilon}=1$.

Proof. This is a simple consequence of Corollary 5.5, since the escape rate for the related induced system, $-\log \Lambda_{\varepsilon}$, is continuous in $\varepsilon$, and by monotonicity, $\bar{\lambda}_{\varepsilon} \geqslant \Lambda_{\varepsilon}$. For details, see the verification of property (P2) in Section 6.1.

Remark 3.4. (Bowen formula for Hausdorff dimension of $\stackrel{\circ}{I}^{\infty}$.) If we take $\phi=-\log |D f|$, then under the assumptions of Theorem 3.1 and for $\varepsilon$ sufficiently small, $\operatorname{Hdim}\left(I^{\infty}\right)=t^{*}$, where $t^{*}$ is the unique value of $t$ such that $P\left(t \phi^{H_{\varepsilon}}\right)=0$. This follows as in [DT1, Theorem 8.1], using the uniform bounds for $t$ close to 1 on the tail of the return time function from Theorem 4.10 to show that any set of Hausdorff dimension greater than some constant $D<1$ lifts to our inducing scheme. Then Theorem 3.1 (c) implies that the dimension of the equilibrium measure $\nu_{H_{\varepsilon}}^{1}$, corresponding to $t=1$, equals $1+\log \lambda_{\varepsilon}$, and so is greater than $D$ for $\varepsilon$ small. Thus the Hausdorff dimension of $i^{\infty}$ equals that of the survivor set in our inducing scheme.

3.2. Zero-hole limits. Here we consider the asymptotic scaling limit for the escape rate, $\frac{-\log \lambda_{\varepsilon}}{\mu_{\varphi}\left(H_{\varepsilon}\right)}$ as $\varepsilon \rightarrow 0$. This limit was first computed in the context of escape rates for full shifts in [BY], then extended to (piecewise) uniformly expanding systems in [KL2] and to more general potentials in the symbolic setting in [FP] (see also [AB, BV2, FFT2]). Its extension to unimodal and multimodal maps followed with added assumptions on the centre of the hole $z$, either assuming that the postcritical orbits approach $z$ slowly [BDM, DT1], or are bounded away from $z$ [PU].

By contrast, for Hölder continuous potentials, we prove our results for all nonperiodic $z \in I$, with an additional assumption required only if $z$ is periodic and lies in the post critical orbit. For geometric potentials, we require a (generic) slow approach condition to $z$, and present an example (Section 3.4 to show that the scaling limit can fail for geometric potentials if no condition on $z$ is imposed. The proofs of Theorems 3.5 and 3.7 are in Section 7.

3.2.1. Hölder potentials. The asymptotic escape rate depends on whether the chosen centre $z$ is periodic or not.

Theorem 3.5. Let $f \in \mathcal{F}, \phi$ be Hölder continuous and $z \in I$.

a) If $z$ is not periodic, then $\lim _{\varepsilon \rightarrow 0} \frac{-\log \lambda_{\varepsilon}}{\mu_{\varphi}\left(H_{\varepsilon}\right)}=1$.

b) If $z$ is periodic with prime period $p$ and $\left\{f^{n}(c): c \in\right.$ Crit, $\left.n \geqslant 1\right\} \cap\{z\}=\emptyset$, then $\lim _{\varepsilon \rightarrow 0} \frac{-\log \lambda_{\varepsilon}}{\mu_{\varphi}\left(H_{\varepsilon}\right)}=1-e^{S_{p} \varphi(z)}$.

c) Suppose $z$ is periodic with prime period $p$ and $\left\{f^{n}(c): c \in\right.$ Crit, $\left.n \geqslant 1\right\} \cap\{z\} \neq \emptyset$. If in addition, either $f^{p}$ is orientation preserving in a neighbourhood of $z$, or $\lim _{\varepsilon \rightarrow 0} \frac{m_{\varphi}(z+\varepsilon, z)}{m_{\varphi}(z, z-\varepsilon)}=$ 1 , then $\lim _{\varepsilon \rightarrow 0} \frac{-\log \lambda_{\varepsilon}}{\mu_{\varphi}\left(H_{\varepsilon}\right)}=1-e^{S_{p} \varphi(z)}$. 
Remark 3.6. Even when both conditions fail in part (c) of Theorem 3.5 fail, we can still find a subsequence of $\varepsilon \rightarrow 0$ so that the scaling limit converges to $1-e^{S_{p} \varphi(z)}$. Thus we expect that the scaling limit holds for all periodic points in the case of Hölder continuous potentials.

3.2.2. Geometric potentials. For the remainder of this section we let $\phi=-\log |D f|$. The geometric case requires a condition on slow approach to the critical set as well as a polynomial rate of growth of the derivative along the post-critical orbit. For simplicity, for a given $d>1$ we will consider the set $\mathcal{F}_{d} \subset \mathcal{F}$ with the defining property that for each $f \in \mathcal{F}_{d}$ all critical points have order $d$.

For $t \in\left(t^{-}, t^{+}\right)$, let $s_{t}:=t+\frac{p_{t}}{\lambda\left(\mu_{t}\right)} \in(0,1]$ denote the local scaling exponent for $m_{t \phi-p_{t}}$, see [DT1, Lemma 9.5]. Define

$$
D_{n}(c)=\left|D f^{n}(f(c))\right| \quad \text { for each } c \in \text { Crit } .
$$

We assume that for each $c \in$ Crit,

$$
D_{n}(c) \geqslant \text { const. } n^{q} \text { for some } q>d+\frac{d-1}{s_{t}} \text { and all } n \geqslant 1 .
$$

With $q$ given as above, we choose $\theta \in(0,1)$ and $r \in\left(\frac{1}{(1-\theta) s_{t}}, \frac{q-d}{d-1}\right)$, and define a sequence $\gamma_{n}=n^{-r}$, $n \geqslant 1$. We make the following assumption on the centre of the hole, $z$, in terms of this sequence:

$$
\exists \delta_{z}>0 \text { s.t. } \min _{c \in \text { Crit }} d\left(f^{n}(c), z\right) \geqslant \delta_{z} \gamma_{n}^{1-\theta}, \quad \text { for all } n \in \mathbb{N} \text {. }
$$

In particular, we have $\sum_{n} \gamma_{n}^{(1-\theta)\left(s_{t}-\epsilon\right)}<\infty$ for some $\epsilon>0$, so that condition 3.3 is generic with respect to the measures $m_{\varphi}, \varphi=t \phi-p_{t}$, as proved in [DT1, Lemma 9.3].

The value of $s_{t}$ varies continuously with $t$, and is $>0$ for each $t \in\left(t^{-}, t^{+}\right)$, with $s_{1}=1$, but may tend to zero as $t$ tends to the boundary of $\left(t^{-}, t^{+}\right)$. This means that in particular when the map $f$ satisfies the $(\mathrm{CE})$ condition, we will restrict to a subinterval $\left(t^{-}, t_{1}\right)$ where $t_{1} \in\left(1, t^{+}\right]$is determined by (7.28); if $f$ does not satisfy (CE), we let $t_{1}=1$.

Theorem 3.7. For $d>1$, let $f \in \mathcal{F}_{d}$ and $t \in\left(t^{-}, t_{1}\right)$. Suppose 3.2 is satisfied and $z \in I$ satisfies (3.3). Then for $\varphi=-t \log |D f|-p_{t}$,

(a) if $z$ is not periodic then $\lim _{\varepsilon \rightarrow 0} \frac{-\log \lambda_{\varepsilon}}{\mu_{\varphi}\left(H_{\varepsilon}\right)}=1$;

(b) if $z$ is periodic with (prime) period $p$, then $\lim _{\varepsilon \rightarrow 0} \frac{-\log \lambda_{\varepsilon}}{\mu_{\varphi}\left(H_{\varepsilon}\right)}=1-e^{S_{p} \varphi(z)}$.

Remark 3.8. [FFT1, Section 6] shows that there are examples of maps $f \in \mathcal{F}_{d}$ and periodic points $z$ satisfying 3.3 .

Remark 3.9. It is not clear what the optimal condition on $z$ is so that the scaling limits of Theorem 3.7 hold, but it is clear that the limits can fail without some assumption on $z$ in the case of geometric potentials. To illustrate this point, we present an example in Section 3.4 using the map $f(x)=4 x(1-x)$ for which (3.3) does not hold, and the relevant scaling limit fails.

3.3. Escape rate function. The asymptotics in the previous section can be seen as a type of derivative of the escape rate at $\varepsilon=0$. Our next result addresses the regularity of the escape rate $-\log \lambda_{\varepsilon}$ from Theorem 3.1 for $\varepsilon>0$.

Theorem 3.10. Let $f \in \mathcal{F}$ and $\phi$ be an admissible potential. Suppose $z \in I$ and let $\varepsilon^{*}>0$ be from Theorem 3.1. Then $\varepsilon \mapsto-\log \lambda_{\varepsilon}$ is continuous on $\left[0, \varepsilon^{*}\right]$ and forms a devil's staircase: $i . e ., \frac{d \log \lambda_{\varepsilon}}{d \varepsilon}$ exists and equals 0 on an open and full measure subset of $\left[0, \varepsilon^{*}\right]$. 
That the escape rate function forms a devil's staircase has been shown in uniformly hyperbolic settings, namely for expanding systems in [KL2], and for Anosov diffeomorphisms in [DW]. The present result is the first in the setting of nonuniformly hyperbolic maps. It stands in contrast to Theorems 3.5 and 3.7, which prove that $\left.\frac{d \log \lambda_{\varepsilon}}{d \varepsilon}\right|_{\varepsilon=0}$ exists and is nonzero. Once Theorem 3.1 is established, it is a direct consequence of the continuity of $f$ and the ergodicity of the measure $\mu_{\varphi}$, so we give this short proof immediately.

Proof of Theorem 3.10. The continuity of $\varepsilon \mapsto-\log \lambda_{\varepsilon}$ follows from Corollary 5.5 and (7.2). We proceed to prove the statement about the derivative of this map. Denote the survivor set by $\stackrel{\circ}{I}_{\varepsilon}^{\infty}=\cap_{n=0}^{\infty} f^{-n}\left(I \backslash H_{\varepsilon}(z)\right)$.

If $\stackrel{\circ}{I}_{\varepsilon}^{\infty} \cap \partial H_{\varepsilon}=\emptyset$, then $\operatorname{dist}\left(\stackrel{I}{I}_{\varepsilon}^{\infty}, \partial H_{\varepsilon}\right)>0$. This follows from the continuity of $f$ and the fact that $H_{\varepsilon}(z)$ is open: If $\stackrel{\circ}{I}_{\varepsilon}^{\infty} \cap \partial H_{\varepsilon}=\emptyset$ then there exists $n>0$ such that $f^{n}(z+\varepsilon) \in H_{\varepsilon}(z)$; by the continuity of $f$, there exists a neighbourhood of $z+\varepsilon, N_{\delta}(z+\varepsilon)$, such that $f^{n}\left(N_{\delta}(z+\varepsilon)\right) \subset H_{\varepsilon}(z)$. A similar argument holds for $z-\varepsilon$.

Thus if $\stackrel{\circ}{I}_{\varepsilon}^{\infty} \cap \partial H_{\varepsilon}=\emptyset$, then ${\stackrel{\circ}{I^{\prime}}}_{\varepsilon^{\prime}}^{\infty} \cap \partial H_{\varepsilon^{\prime}}=\emptyset$ for all $\varepsilon^{\prime} \in\left(\varepsilon-\delta^{\prime}, \varepsilon+\delta^{\prime}\right)$ for some $\delta^{\prime}>0$, i.e. the fact that the boundary of the hole falls into the hole is an open condition. It follows from this that $\stackrel{\circ}{I}_{\varepsilon}^{\infty}={\stackrel{\circ}{I_{\varepsilon^{\prime}}}}_{\infty}$ for all $\varepsilon^{\prime} \in\left(\varepsilon-\delta^{\prime}, \varepsilon+\delta^{\prime}\right)$, and thus that $P\left(\varphi^{H_{\varepsilon}}\right)=P\left(\varphi^{H_{\varepsilon^{\prime}}}\right)$ and by Theorem 3.1(c), $\lambda_{\varepsilon}=\lambda_{\varepsilon^{\prime}}$ for all $\varepsilon^{\prime} \in\left(\varepsilon-\delta^{\prime}, \varepsilon+\delta^{\prime}\right)$.

Thus $\log \lambda_{\varepsilon}$ is locally constant whenever $\stackrel{\circ}{I}_{\varepsilon}^{\infty} \cap \partial H_{\varepsilon}=\emptyset$.

Finally, since $\mu_{\varphi}\left(H_{\varepsilon}\right)>0$, ergodicity implies that generic $z \pm \varepsilon$ fall in the hole, so the condition $\stackrel{\circ}{I}_{\varepsilon}^{\infty} \cap \partial H_{\varepsilon}=\emptyset$ is generic. Therefore,

$$
\mu_{\varphi}\left\{x=z+\varepsilon \in I: \varepsilon \in\left(0, \varepsilon^{*}\right) \text { and } \frac{d \log \lambda_{\varepsilon}}{d \varepsilon} \neq 0\right\}=0
$$

as required.

3.4. An example of scaling limit failure. In this section we present an example of a map in our class $\mathcal{F}$ and choice of $z$ such that condition $(3.3)$ is violated and the conclusion of Theorem 3.7 fails.

Let $f: I \circlearrowleft$ be defined by $f(x)=4 x(1-x)$. Let $X$ also denote the unit interval, and $T: X \circlearrowleft$ be the tent map $T(x)=2 x, x \in[0,1 / 2]$, and $T(x)=2(1-x), x \in[1 / 2,1]$.

The well-known conjugacy between $f$ and $T$ is $g: X \rightarrow I, g(x)=\sin ^{2}\left(\frac{\pi x}{2}\right)$, so that $f \circ g(x)=g \circ T(x)$ for all $x \in X$.

Let $m$ denote Lebesgue measure on $X$, which is $T$-invariant and the equilibrium state for the potential $-\log |D T|$. The absolutely continuous invariant probability measure for $f$ can then be written as $\mu=g_{*} m$, which is the equilibrium state for the potential $-\log |D f|$.

We choose $z=0$, a fixed point for $f$, and define $H_{\varepsilon}=[0, \varepsilon)$. It is clear that (3.3) fails, since Crit $=\left\{\frac{1}{2}\right\}$ and $f^{2}\left(\frac{1}{2}\right)=0$.

Now $g^{-1}\left(H_{\varepsilon}\right)=\left[0, \varepsilon^{\prime}\right)$, where $\varepsilon^{\prime}=\frac{2}{\pi} \sin ^{-1}(\sqrt{\varepsilon})$. Note that since $X=g^{-1}(I)$, we have

$$
\begin{aligned}
m\left(\dot{X}^{n}\right) & :=m\left(\cap_{i=0}^{n} T^{-i}\left(X \backslash g^{-1}\left(H_{\varepsilon}\right)\right)=m\left(\cap_{i=0}^{n} T^{-i}\left(g^{-1}\left(I \backslash H_{\varepsilon}\right)\right)\right)\right. \\
& =m\left(\cap_{i=0}^{n} g^{-1} \circ f^{-i}\left(I \backslash H_{\varepsilon}\right)\right)=m\left(g^{-1}\left(\cap_{i=0}^{n} f^{-i}\left(I \backslash H_{\varepsilon}\right)\right)\right)=\mu\left(I^{n}\right),
\end{aligned}
$$


where $\dot{X}^{n}$ and $\stackrel{\circ}{I}^{n}$ denote the $n$-step survivor sets for $T$ and $f$, respectively.

Thus the escape rate $-\log \lambda_{\varepsilon}$ for $\left(f, \mu, H_{\varepsilon}\right)$ is the same as the escape rate for $\left(T, m, g^{-1}\left(H_{\varepsilon}\right)\right)$.

Now applying [BY, Theorem 4.6.1 and Section 5] (see also [KL2, Theorem 2.1 and Section 3.1]) to $T$, we compute the scaling limit,

$$
\lim _{\varepsilon \rightarrow 0} \frac{-\log \lambda_{\varepsilon}}{\mu\left(H_{\varepsilon}\right)}=\lim _{\varepsilon \rightarrow 0} \frac{-\log \lambda_{\varepsilon}}{m\left(g^{-1}\left(H_{\varepsilon}\right)\right)}=1-\frac{1}{D T(0)}=\frac{1}{2} .
$$

Yet $D f(0)=4$, so that the expected scaling limit for $f$ would be $1-\frac{1}{D f(0)}=\frac{3}{4} \neq \frac{1}{2}$.

Remark 3.11. Although the scaling limit of Theorem 3.7 fails in this case, we note that an alternate formulation is possible. Indeed, the invariant density for $f$ with respect to Lebesgue measure has a spike of order $x^{-1 / 2}$ at $z=0$. So the limit of $\frac{1}{2}$ that we compute is compatible with the formula,

$$
\lim _{\varepsilon \rightarrow 0} \frac{-\log \lambda_{\varepsilon}}{\mu\left(H_{\varepsilon}\right)}=1-\left(\frac{1}{D f(0)}\right)^{1 / 2}=1-\left(\frac{1}{4}\right)^{1 / 2}=1-\frac{1}{2}=\frac{1}{2},
$$

where the scaling exponent of $1 / 2$ matches the exponent in the spike of the invariant density. Such relations follow from O'Brien's formula for the extremal index (see [FFT2, (2.6)] for a dynamical setting of this), and given the connection between extremal indices and scaling limits for escape rates established in [BDT], we conjecture that it holds in greater generality for scaling limits.

\section{Construction of EXtensions And PREliminary Results}

4.1. Distortion and contraction. As is standard in this field we wish to recover some uniform expansion and uniform distortion from a system which is non-uniformly hyperbolic. We will use versions of the Koebe Lemma often, so state it here (see [MS, Theorem IV.1.2]) recalling that elements of $\mathcal{F}$ have negative Schwarzian derivative.

Lemma 4.1 (Koebe Lemma). For any $\epsilon>0$, there exists $K(\epsilon) \geqslant 1$ such that the following hold. If $f \in \mathcal{F}$ and $U \Subset U^{\prime}$ is such that $U^{\prime} \backslash U$ consists of two intervals length $\geqslant \epsilon|U|$ and $f^{n}: U^{\prime} \rightarrow f^{n}\left(U^{\prime}\right)$ is a diffeomorphism then,

(a) for $x, y \in U$,

$$
\frac{D f^{n}(x)}{D f^{n}(y)} \leqslant K(\epsilon)
$$

(b) for $x, y \in U$,

$$
\left|\frac{D f^{n}(x)}{D f^{n}(y)}-1\right| \leqslant K(\epsilon) \frac{|x-y|}{|U|} .
$$

For expansion/backward contraction we use 'polynomial shrinking'. That is, for $\beta>0$,

- $(\text { PolShr })_{\beta}$ : there are constants $\delta, C>0$ such that for each $x \in I$ and every integer $n \geqslant 1$, any connected component $W$ of $f^{-n}\left(B_{\delta}(x)\right)$ has $|W| \leqslant C n^{-\beta}$.

Combining [R-LS, Theorem A] and [BRSS, Theorem 1], for each $f \in \mathcal{F}$ this holds for any $\beta>0 .^{2}$ Notice that for intervals of size larger than $\delta$ in our setting, we can simply chop these up into smaller intervals at the cost of adding a multiplicative constant.

\footnotetext{
${ }^{2}$ In fact, these results imply that to obtain (PolShr $)_{\beta}$ for a particular $\beta$, one does not need $\left|D f^{n}(f(c))\right| \rightarrow \infty$ for all $c \in$ Crit, but rather a specific lower bound for $\left|D f^{n}(f(c))\right|$ depending on $\beta$ suffices.
} 
4.2. Hofbauer extensions. Hofbauer extensions are Markov extensions of $f: I \circlearrowleft$ usually defined by introducing cuts at (images of) critical points, but in fact we can cut at arbitrary points: in Section 4.4 we will give a definition of our 'extended critical set'. So we let $\mathcal{C} r \subset I$ be a finite set of points with Crit $\subset \mathcal{C} r$. Set $\mathcal{P}_{0}:=I$, let $\mathcal{P}_{1}$ be the partition defined by $\mathcal{C} r$, and define $n$-cylinders by

$$
\mathcal{P}_{n}:=\bigvee_{i=0}^{n-1} f^{-i} \mathcal{P}_{1}
$$

We will denote the $n$-cylinder which $x \in I$ lies in by $Z_{n}[x]$ (note that if there are two, then we can make an arbitrary choice). Now define $\mathcal{D}:=\left\{f^{k}(Z): Z \in \mathcal{P}_{k}, k \geqslant 0\right\}$. As $\mathcal{D}$ is a set, each element $D \in \mathcal{D}$ appears once (i.e., if $f^{k}(Z)=f^{j}\left(Z^{\prime}\right)$ then these elements are naturally identified as the same set). The Hofbauer extension is defined as the disjoint union

$$
\hat{I}=\hat{I}(\mathcal{C} r):=\sqcup_{D \in \mathcal{D}} D .
$$

We call each $D$ a domain of $\hat{I}$. There is a natural projection map $\pi: \hat{I} \rightarrow I$, so each point $\hat{x} \in \hat{I}$ can be represented as $(x, D)$ where $x=\pi \hat{x}$. The map $\hat{f}: \hat{I} \circlearrowleft$ is defined by $\hat{f}(\hat{x})=\hat{f}(x, D)=\left(f(x), D^{\prime}\right)$ if there are cylinder sets $Z^{\prime} \subset Z$ with $Z^{\prime} \in \mathcal{P}_{k+1}$ and $Z \in \mathcal{P}_{k}$ such that

$$
x \in f^{k}\left(Z^{\prime}\right) \subset f^{k}(Z)=D \text { and } D^{\prime}=f^{k+1}\left(Z^{\prime}\right) .
$$

In this case we write $D \rightarrow D^{\prime}$, so $(\mathcal{D}, \rightarrow)$ has the structure of a directed graph. With this setup, $\pi$ acts as a semiconjugacy between $\hat{f}$ and $f$ :

$$
\pi \circ \hat{f}=f \circ \pi .
$$

We can think of points in $\mathcal{C} r$ as 'cut points' since if an open interval $\hat{A}=(A, D) \subset \hat{I}$ and $\#\{A \cap \mathcal{C} r\}=$ $k \geqslant 1$, then $\hat{A}$ gets cut at each element of $\mathcal{C} r$ (strictly speaking, of $\pi^{-1}(\mathcal{C} r)$ ) so that $\hat{f}(\hat{A})$ lies in $k+1$ different elements of $\mathcal{D}$.

Let $D_{0}$ be the base of $\hat{I}$, that is the copy of $I$ in the extension. Define $\iota$ to be the natural inclusion map sending $I$ to $D_{0}$. For $D \in \mathcal{D}$ we let level $(D)$ be the length of the shortest path $D_{0} \rightarrow \cdots \rightarrow D$ in $(\mathcal{D}, \rightarrow)$. Then for $L \in \mathbb{N}$, the truncated extension at level $L$ is

$$
\hat{I}(L):=\sqcup\{D \in \mathcal{D}: \operatorname{level}(D) \leqslant L\} .
$$

The following lemma and proof are well-known in the area, but we include them for illustrative purposes and for use later.

Lemma 4.2. Suppose that $\hat{x}, \hat{y} \in \hat{I} \backslash \partial \hat{I}$ have $\pi \hat{x}=\pi \hat{y}$. Then there exists $n \in \mathbb{N}$ such that $\hat{f}^{n}(\hat{x})=\hat{f}^{n}(\hat{y})$.

Proof. Let $w=\pi \hat{x}$. Observe that since $\pi$ is a semiconjugacy, $\hat{f}^{k}(\hat{x}), \hat{f}^{k}(\hat{y}) \in \pi^{-1}\left(f^{k}(w)\right)$ for all $k \geqslant 0$. Let $D_{\hat{x}}$ and $D_{\hat{y}}$ denote the domains of $\hat{I}$ which contain $\hat{x}$ and $\hat{y}$ respectively. Then choose $n$ so large that $\left(\left.\pi\right|_{D_{\hat{x}}}\right)^{-1}\left(Z_{n}[w]\right)$ and $\left(\left.\pi\right|_{D_{\hat{y}}}\right)^{-1}\left(Z_{n}[w]\right)$ are both compactly contained inside $D_{\hat{x}}$ and $D_{\hat{y}}$ respectively, where $Z_{n}[w]$ denotes the element of $\mathcal{P}_{n}$ containing $w$. Now notice that $f^{n}\left(Z_{n}[w]\right)$ is a domain of the Hofbauer extension, and indeed it follows from the construction in (4.1) that $\hat{f}^{n}(\hat{x})$ and $\hat{f}^{n}(\hat{y})$ must lie in $f^{n}\left(Z_{n}[w]\right)$. Since these iterates must also both lie on the fibre $\pi^{-1}\left(f^{n}(w)\right)$ by the conjugacy property, the points must coincide, as required.

In general, Hofbauer extensions split into a collection of transitive components and a non-transitive set, see [HR], but the above lemma and the leo property imply that there is a unique transitive component. Since any points outside this must map into it and stay there forever, we will adopt the convention that $\hat{I}(L)$ is always restricted to the transitive component. 
Given a set $A \subset I$, the set $\hat{A}=\pi^{-1}(A)$ is called the lift of $A$. We now consider how to lift measures to $\hat{I}$. Suppose that $\mu$ is an ergodic $f$-invariant probability measure. Set $\hat{\mu}^{(0)}:=\mu \circ \iota^{-1}$, and for $n \in \mathbb{N}$,

$$
\hat{\mu}^{(n)}:=\frac{1}{n} \sum_{k=0}^{n-1} \hat{\mu}^{(0)} \circ \hat{f}^{-k} .
$$

As in $[\mathrm{K}]$, if $h_{\mu}(f)>0$, then $\hat{\mu}^{(n)}$ converges in the vague topology ${ }^{3}$ to $\hat{\mu}$, which is an $\hat{f}$-invariant ergodic measure with

Also, $[\mathrm{K}]$ shows that $h_{\hat{\mu}}(\hat{f})=h_{\mu}(f)$.

$$
\hat{\mu} \circ \pi^{-1}=\mu \text {. }
$$

We will also be interested in lifting conformal measures. Given a conformal measure $m_{\phi}$ on $I$, define $\hat{m}_{\phi}:=m_{\phi} \circ \pi^{-1}$. Clearly $\hat{m}_{\phi}$ is $\hat{\phi}$-conformal for $\hat{\phi}:=\phi \circ \pi$ on $\hat{I}$. Note that in general it could be the case that $\hat{m}_{\phi}(\hat{I})=\infty$.

Remark 4.3. We can define pressure $P(\hat{\phi})$ analogously to (2.1). As in (2.3), for admissible potentials we need only consider measures with positive entropy, so we deduce that $P(\hat{\phi})=P(\phi)$. This implies that when we lift the normalised potential, $\hat{\varphi}:=\varphi \circ \pi$, then the relation $\hat{\varphi}=\hat{\phi}-P(\hat{\phi})$ continues to hold.

4.3. Inducing schemes. We wish to define inducing schemes via first return maps to truncated domains in the Hofbauer extension, whose partition we will refine further below: it will also be useful to set this up for our punctured systems, though there will be a small difference in the structure there. To this end, let $\hat{\mathcal{P}}_{n}$ be the set of intervals $\left\{\left(\left.\pi\right|_{D}\right)^{-1}(Z): D \in \mathcal{D}, Z \in \mathcal{P}_{n}\right\}$. For a domain $D \in \mathcal{D}$, let $D_{\ell}^{L}$ be the left-most interval of $\hat{\mathcal{P}}_{L}$ in $D$ and $D_{r}^{L}$ be the right-most,

$$
\hat{I}^{\prime}(L):=\hat{I}(L) \cap\left[\sqcup_{D \in \mathcal{D}}\left(D \backslash\left(D_{\ell}^{L} \cup D_{r}^{L}\right)\right)\right] .
$$

It follows, for example from [DoT, Lemma 8.2] that, so long as $\hat{I}$ has more than one domain, then for all $\epsilon>0$ there exists $L \in \mathbb{N}$ such that if $h_{\mu}(f)>\epsilon$ then $\hat{\mu}\left(\hat{I}^{\prime}(L)\right)>0$.

We further partition $\hat{I}^{\prime}(L)$ into the elements of $\hat{\mathcal{P}}_{L}$ intersecting it and denote this collection by $\mathcal{Q}$, (i.e., $\mathcal{Q}=\left\{Q \in \hat{\mathcal{P}}_{L}: Q \subset \hat{I}^{\prime}(L)\right\}$ ), see Figure 1. Letting $R$ be the first return time to $Y:=\hat{I}^{\prime}(L)$, the map $F=\hat{f}^{R}$ is the first return map. We denote the domains of $F$ by $\left\{Y_{i}\right\}_{i}$. These are the maximal sets $U$ such that $U \subset Q$ and $F(U) \subset Q^{\prime}$ for some $Q, Q^{\prime} \in \mathcal{Q}$, so that $F$ is monotonic and $R$ is constant on $U$. We set $R_{i}=\left.R\right|_{Y_{i}}$. The cylinder structure of $\mathcal{Q}$ ensures that the $\left\{Y_{i}\right\}_{i}$ are disjoint and the Markov structure ensures that the image of such a domain is an interval $Q$ of $\mathcal{Q}$, see [DoT, Lemma 4.9]. We give a short proof of this fact to explain how the changes we make later will not affect this structure.

Lemma 4.4 (Markov property of $F$ ). If $Y_{i}$ is a domain of $F$ with $F\left(Y_{i}\right) \subset Q \in \mathcal{Q}$ then $F\left(Y_{i}\right)=Q$.

Proof. Let $D \in \mathcal{D}$ denote the domain in which $Y_{i}$ lies and suppose $R_{i}=n$. By the Markov structure of the Hofbauer extension there must exist $Y_{i}^{\prime} \subset D$ such that $\hat{f}^{n}\left(Y_{i}^{\prime}\right)=Q$. If $Y_{i} \neq Y_{i}^{\prime}$ then the only constraint that $Y_{i}$ must satisfy which $Y_{i}^{\prime}$ does not need to is that $Y_{i}$ must be contained in some $Q^{\prime} \in \hat{\mathcal{P}}_{L}$. This means that $Y_{i}$ must have an element of $\partial \hat{\mathcal{P}}_{L}$ as a boundary point: indeed it must be adjacent to some $D_{\ell}^{L}$ or $D_{r}^{L}$. Denote such a point by $a_{-j}$ where $\pi\left(a_{-j}\right) \in f^{-j} \mathcal{C} r$. In particular $j \leqslant L$. So if $n>j$ then in fact $\hat{f}^{n}\left(a_{-j}\right)$ must be a boundary point of some $D \in \mathcal{D}$, which is a

\footnotetext{
${ }^{3}$ Recall that $\hat{\mu}^{(n)}$ converges to $\hat{\mu}$ vaguely if $\hat{\mu}^{(n)}(\psi)$ converges to $\hat{\mu}(\psi)$ for all continuous $\psi$ with compact support in $\hat{I}$.
} 


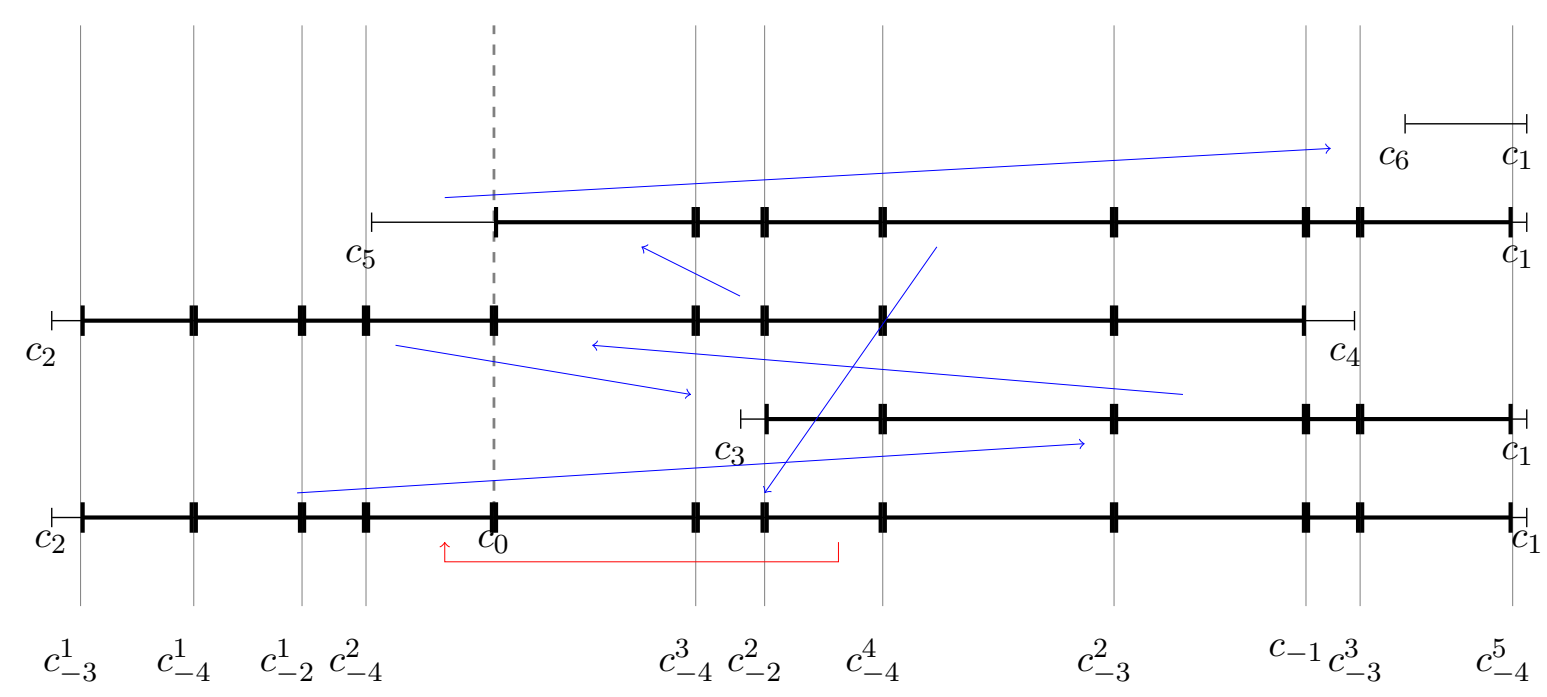

Figure 1. A sketch of the first few levels of a Hofbauer extension for a unimodal map. The dotted line shows where we cut at the critical point $c_{0}$, a blue arrow shows movement between domains in different levels and a red arrow shows movement between domains in the same level (the colouring will be most useful when we have extra cuts, as we will later). We denote by $c_{n}=f^{n}\left(c_{0}\right)$. We also indicate the boundaries of the cylinder sets, denoted $c_{-j}^{i}$, in $\mathcal{P}_{4}$, and draw vertical lines to indicate how this lifts to $\hat{\mathcal{P}}_{4}$. Thick vertical lines imply these points are doubled. These endpoints are then used to determine $\hat{I}^{\prime}(4)$, as well as the domains $Q$ of $Y$, which are drawn with thick black lines.

contradiction. On the other hand if $n \leqslant j$ then $\hat{f}^{n}\left(a_{-j}\right)$ is a boundary point of an element of $\hat{\mathcal{P}}_{L}$ so in fact $\hat{f}^{n}\left(Y_{i}\right)=Q$ and $Y_{i}=Y_{i}^{\prime}$.

Remark 4.5. In the construction above, we used $\hat{\mathcal{P}}_{L}=\hat{\mathcal{P}}_{L}(\mathcal{C} r)$ to firstly arrange for $\hat{I}(L)$ to be trimmed to $\hat{I}^{\prime}(L)$ and then secondly to partition the domains of $\hat{I}^{\prime}(L)$ into $\mathcal{Q}$. We observe here that if a subset $\mathcal{C} r^{\prime} \subset \mathcal{C} r$ is instead used to produce $\hat{\mathcal{P}}_{L}\left(\mathcal{C} r^{\prime}\right)$ and this set used in place of $\hat{\mathcal{P}}_{L}(\mathcal{C} r)$, the setup above, and in particular the conclusion of Lemma 4.4, still holds. We will employ such a construction in Section 4.4.

Note that the set of domains generate a cylinder structure for $F$, which we will denote by $\left\{Y_{i}^{(n)}\right\}_{i}$ for the collection of $n$-cylinders. The Markov structure of the Hofbauer extension implies for that each domain of $F$, if it maps onto $D(L) \in \hat{I}^{\prime}(L)$, where $D(L) \subset D \in \mathcal{D}$, then there is an extension so that $F$ extends to a map onto $D$. As in Lemma 4.1, this extension property gives us bounded distortion for $F$ : there exists $K \geqslant 1$ such that for $Y_{i}$ a domain of $F$, if $x, y \in Y_{i}$ then

$$
\frac{|D F(x)|}{|D F(y)|} \leqslant K
$$

(we improve on this estimate in Lemma 4.6). Note that $K$ depends on $L$ since $L$ determines the constant $\epsilon$ in Lemma 4.1 ,

We also note that by [DoT, Lemma 10.7], $F$ is uniformly hyperbolic, i.e., there exist $C_{F}>0$ and $\sigma_{F}>1$ such that for $x \in Y$ and any $n \geqslant 1$,

$$
\left|D F^{n}\right| \geqslant C_{F} \sigma_{F}^{n} \text {. }
$$


Given a potential $\phi: I \rightarrow[-\infty, \infty]$, and its normalised lift $\hat{\varphi}=\varphi \circ \pi$ as in Remark 4.3, we define the induced potential

$$
\Phi(x)=\hat{\varphi}(x)+\hat{\varphi}(\hat{f}(x))+\cdots+\hat{\varphi}\left(\hat{f}^{R(x)}(x)\right), \quad x \in \hat{I}^{\prime}(L) .
$$

As in 2.2, if $\hat{m}_{\varphi}$ is $\hat{\varphi}$-conformal for $\hat{f}$, then it is also $\Phi$-conformal for $F$.

By Kac's Lemma, since $F$ is a first return map to $Y$, if $\hat{\mu}$ is a $\hat{f}$-invariant probability measure then

$$
\hat{\mu}_{Y}=\frac{\left.\hat{\mu}\right|_{Y}}{\hat{\mu}(Y)} \text { is an } F \text {-invariant probability measure and } \hat{\mu}(Y)=\frac{1}{\int R d \hat{\mu}_{Y}} \text {. }
$$

We also note that

$$
\hat{\mu}(A)=\hat{\mu}(Y) \sum_{i} \sum_{j=0}^{R_{i}-1} \hat{\mu}_{Y}\left(Y_{i} \cap \hat{f}^{-j} A\right)=\sum_{i} \sum_{j=0}^{R_{i}-1} \hat{\mu}\left(Y_{i} \cap \hat{f}^{-j} A\right), \text { for any Borel } A \subset \hat{I},
$$

where the sum over $i$ is taken over all 1-cylinders $Y_{i}$ for $F$, and $R_{i}=\left.R\right|_{Y_{i}}$.

We close this subsection with the following distortion result, which is primarily due to Lemma 4.1

Lemma 4.6. (a) Suppose that $\phi: I \rightarrow \mathbb{R}$ is Hölder continuous with Hölder exponent $\eta \leqslant 1$. Then there exists $K_{F, \phi}>0$ such that for any $n$-cylinder $Y_{i}^{(n)}$, and all $x, y \in Y_{i}^{(n)}$,

$$
\left|S_{n} \Phi(x)-S_{n} \Phi(y)\right| \leqslant K_{F, \phi}\left|F^{n}(x)-F^{n}(y)\right|^{\eta} .
$$

(b) There exists $K_{F}>0$ such that for any $n$-cylinder of the scheme $Y_{i}^{(n)}$, and all $x, y \in Y_{i}^{(n)}$,

$$
\left|\frac{D F^{n}(x)}{D F^{n}(y)}-1\right| \leqslant K_{F}\left|F^{n}(x)-F^{n}(y)\right| .
$$

Proof. We prove (a) first. We begin by taking a 1-cylinder $Y_{i}$ and $x, y \in Y_{i}$. Then

$$
\begin{aligned}
|\Phi(x)-\Phi(y)| & \leqslant \sum_{k=0}^{R_{i}-1}\left|\phi \circ f^{k}(x)-\phi \circ f^{k}(y)\right| \leqslant C \sum_{k=0}^{R_{i}-1}\left|f^{k}(x)-f^{k}(y)\right|^{\eta} \\
& =C \sum_{k=0}^{R_{i}-1}\left(\frac{\left|f^{k}(x)-f^{k}(y)\right|}{|F(x)-F(y)|}\right)^{\eta}|F(x)-F(y)|^{\eta} \leqslant K C|F(x)-F(y)|^{\eta} \sum_{k=0}^{R_{i}-1}\left(\frac{\left|f^{k}\left(Y_{i}\right)\right|}{\left|f^{R_{i}}\left(Y_{i}\right)\right|}\right)^{\eta},
\end{aligned}
$$

where $K$ is a distortion constant coming from Lemma 4.1. So for a Hölder condition on the induced potential it suffices to have a bound on $\sum_{k=0}^{R_{i}-1}\left(\frac{\left|f^{k}\left(Y_{i}\right)\right|}{\left|f^{R}\left(Y_{i}\right)\right|}\right)^{1}$, which follows from (PolShr $)_{\beta}$ for $\beta>1 / \eta$.

Note that since $F$ is uniformly hyperbolic as in 4.3 , this result passes to $n$-cylinders, proving (a).

Part (b) is an immediate consequence of Lemma 4.1(b). Note that when considering a cylinder $Y_{i}^{(n)}$, the switch from $\frac{|x-y|}{\left|Y_{i}^{(n)}\right|}$ to $\left|F^{n}(x)-F^{n}(y)\right|$ follows by Lemma 4.1 (a) and that $\left|F^{n}\left(Y_{i}^{(n)}\right)\right| \asymp 1$.

Remark 4.7. The above lemma, Remark 4.3 and the proof of [DT2, Propostion 1.6] imply that for admissible normalised potentials $\varphi$, the induced potential $\Phi$ has $P(\Phi)=0$ where pressure for the induced system is defined analogously to (2.1). 
4.4. Punctured extensions with uniform images and uniform tails. In order to study open systems via the Hofbauer extension, once we fix a point $z \in I$ to be the centre of our hole, we will introduce extra cuts during the construction of the extension. Indeed, in order to compare Hofbauer extensions with different sets of cuts in a neighbourhood of $z$, we will construct extensions with uniform images for the induced maps that are independent of these extra cuts.

Our notation is the following. For $\varepsilon_{0}>0$ to be chosen below and $0<\varepsilon<\varepsilon_{0}$, we will construct two related Hofbauer extensions: $\hat{I}_{z, \varepsilon_{0}}$ introducing cuts at $z$ and $z \pm \varepsilon_{0}$; and $\hat{I}_{z, \varepsilon_{0}, \varepsilon}$ introducing cuts at $z, z \pm \varepsilon_{0}$, and $z \pm \varepsilon$. In particular, this means that we will add $f^{-1}(z), f^{-1}\left(z \pm \varepsilon_{0}\right)$ and $f^{-1}(z \pm \varepsilon)$ to our critical set. The corresponding dynamics are denoted by $\hat{f}_{z, \varepsilon_{0}}$ and $\hat{f}_{z, \varepsilon_{0}, \varepsilon}$, respectively. A simplified diagram is presented in Figure 2.

We fix $z$ and at the beginning of Section 4.5, we will choose the relevant quantities in the following order. First, we will choose $L$ according to Theorem 4.10, which will provide uniform control on the complexity of the tail of the Hofbauer extension and will depend only on the cardinality of the critical set plus $5 \operatorname{deg}(f)$. Next, we will choose $\varepsilon_{0}^{*}$ according to 4.6 , then finally we choose $\varepsilon_{0} \leqslant \varepsilon_{0}^{*}$, which will fix the return domain $Y$, and work with $0<\varepsilon<\varepsilon_{0}$ as the variable size of the hole.

$\hat{I}_{z}=\hat{I}\left(\mathrm{Crit}_{z}\right)$. Let $\mathrm{Crit}_{z}$ denote the expanded critical set, i.e., Crit $\cup\left\{f^{-1} z\right\}$. Next, consider the $\overline{\text { partition } \mathcal{P}_{L}}=\mathcal{P}_{L}\left(\mathrm{Crit}_{z}\right)$ of $I$ into $L$-cylinders with endpoints at $\left\{f^{-j}(y): y \in \mathrm{Crit}_{z}, 0 \leqslant j \leqslant L\right\}$. We choose

$$
\varepsilon_{0}^{*}<\frac{1}{\left|D f^{L}\right|_{\infty}} \min \left\{|x-y|: x \neq y, x \in \partial \mathcal{P}_{L}, y \in f^{j}\left(\mathrm{Crit}_{z}\right), 0 \leqslant j \leqslant L\right\} .
$$

$\hat{I}_{z, \varepsilon_{0}}=\hat{I}\left(\operatorname{Crit}_{z, \varepsilon_{0}}\right)$. For $0<\varepsilon_{0} \leqslant \varepsilon_{0}^{*}$, we define $\hat{I}_{z, \varepsilon_{0}}=\hat{I}\left(\right.$ Crit $\left._{z, \varepsilon_{0}}\right)$ as above where Crit $_{z, \varepsilon_{0}}$ has

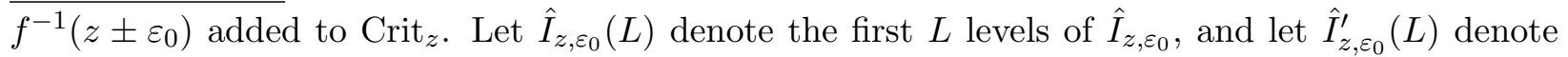
$\hat{I}_{z, \varepsilon_{0}}(L)$ minus the elements of $\hat{\mathcal{P}}_{L}\left(\mathrm{Crit}_{z, \varepsilon_{0}}\right)$ adjacent to each boundary point in $\hat{I}_{z, \varepsilon_{0}}(L)$, as in 4.2 , so that the new boundary points are of the form $f^{-j}(y)$ for some $y \in \mathrm{Crit}_{z, \varepsilon_{0}}$ and $0 \leqslant j \leqslant L$. Note that by choice of $\varepsilon_{0}^{*}$, we completely remove elements of the form $\left[f^{k}(z), f^{k}\left(z+\varepsilon_{0}\right)\right]$ for $0 \leqslant k \leqslant L$, and analogues, in going from $\hat{I}_{z, \varepsilon_{0}}(L)$ to $\hat{I}_{z, \varepsilon_{0}}^{\prime}(L)$.

$\hat{I}_{z, \varepsilon_{0}, \varepsilon}=\hat{I}\left(\operatorname{Crit}_{z, \varepsilon_{0}, \varepsilon}\right)$. For any $\varepsilon \in\left(0, \varepsilon_{0}\right)$, we define $\operatorname{Crit}_{z, \varepsilon_{0}, \varepsilon}$ to be $\operatorname{Crit}_{z, \varepsilon_{0}}$ with $f^{-1}(z \pm \varepsilon)$ added. Let $\hat{I}_{z, \varepsilon_{0}, \varepsilon}=\hat{I}\left(\operatorname{Crit}_{z, \varepsilon_{0}, \varepsilon}\right)$ and define $\hat{I}_{z, \varepsilon_{0}, \varepsilon}^{\prime}(L)$ to be the first $L$ levels, $\hat{I}_{z, \varepsilon_{0}, \varepsilon}(L)$, minus the elements of $\hat{\mathcal{P}}_{L}\left(\right.$ Crit $\left._{z, \varepsilon_{0}, \varepsilon}\right)$ adjacent to each boundary point in $\hat{I}_{z, \varepsilon_{0}, \varepsilon}(L)$ so that the new boundary points are of the form $f^{-j}(y)$ for some $y \in \mathrm{Crit}_{z, \varepsilon_{0}, \varepsilon}$ and $0 \leqslant j \leqslant L$. As above, we completely remove elements of the form $\left[f^{k}(z), f^{k}\left(z+\varepsilon_{0}\right)\right]$ for $0 \leqslant k \leqslant L$, and analogues, in going from $\hat{I}_{z, \varepsilon_{0}, \varepsilon}(L)$ to $\hat{I}_{z, \varepsilon_{0}, \varepsilon}^{\prime}(L)$.

As can be seen from this construction, the domains of $\hat{I}_{z, \varepsilon_{0}}^{\prime}(L)$ and $\hat{I}_{z, \varepsilon_{0}, \varepsilon}^{\prime}(L)$ are the same. We choose $\mathcal{Q}$ to be the domains of $\hat{I}_{z, \varepsilon_{0}, \varepsilon}^{\prime}(L)$ further partitioned by $\hat{\mathcal{P}}_{L}\left(\mathrm{Crit}_{z, \varepsilon_{0}}\right)$. We choose this partition rather than $\hat{\mathcal{P}}_{L}\left(\right.$ Crit $\left._{z, \varepsilon_{0}, \varepsilon}\right)$ to ensure our $F$-images have size independent of $\varepsilon$ and because, as in Remark 4.5, this does not affect the Markov structure for $F$ since the extra cuts due to $\varepsilon$ fall within intervals of the form $\left[f^{k}(z), f^{k}\left(z+\varepsilon_{0}\right)\right]$ for $0 \leqslant k \leqslant L$, which have already been removed from $\hat{I}_{z, \varepsilon_{0}, \varepsilon}^{\prime}(L)$.

Remark 4.8. Here we explain how cutting at $f^{-1}(z)$ and our choice of $\varepsilon_{0}^{*}$ ensures that the representatives of the holes in the Hofbauer extension are disjoint from our inducing domains.

(a) If $\hat{f}: U \rightarrow D, D \in \mathcal{D}$, is a homeomorphism, then since we cut at $f^{-1}(z)$, the interior of $U$ cannot intersect $\pi^{-1}\left(f^{-1}(z)\right)$, which also implies that the interior of $D$ cannot intersect 


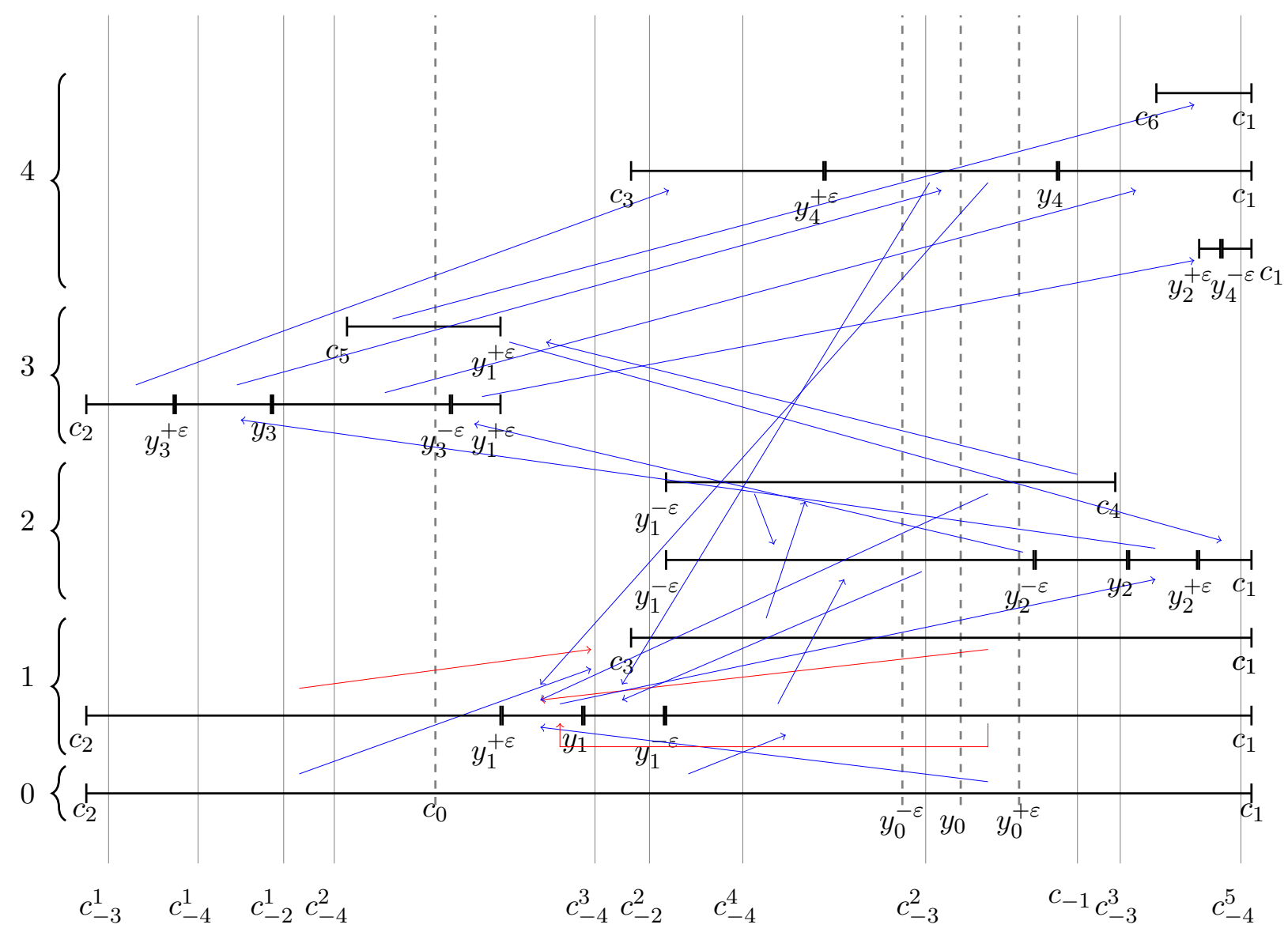

Figure 2. The Hofbauer extension based on the same unimodal map as in Figure1. but with new cuts at points $y_{0}^{+\varepsilon}, y_{0}$ and $y_{0}^{-\varepsilon}$. These represent one set of preimages of $f^{-1}\left(z-\varepsilon_{0}\right), f^{-1}(z)$ and $f^{-1}\left(z+\varepsilon_{0}\right)$ : adding in all preimages adds to the complexity of the diagram significantly. Similarly for simplicity we include only the preimages of $c_{0}$ at the bottom of the diagram, and omit the preimages of $y_{0}^{+\varepsilon}, y_{0}$ and $y_{0}^{-\varepsilon}$ (therefore unlike in Figure 1 we do not mark out the domains $Q$ of $Y$ here). In levels above 0 any marked point is a boundary point of $\mathcal{D}$ : thicker markers imply that these points are doubled. Since, in contrast to Figure1, the number of domains of a given level can be greater than one, we add in the numbers on the left to clarify the level of each domain. Note that with $\varepsilon_{0}$ fixed less than $\varepsilon_{0}^{*}$, additional cuts can be introduced at $z \pm \varepsilon$ for $\varepsilon<\varepsilon_{0}$ which do not affect the structure of the cylinders outside the intervals $\left(y_{n}, y_{n}^{+\varepsilon}\right)$ and $\left(y_{n}^{-\varepsilon}, y_{n}\right)$.

$\pi^{-1}(z)$. Therefore, this fact must be true for any $D$ in the transitive part of $\hat{I}_{z}$. So we conclude that $\pi^{-1}(z) \cap \hat{I}_{z}^{\prime}(L)=\emptyset$ due to trimming of $L$-cylinders.

(b) Suppose that $\hat{J}_{\varepsilon_{0}}(z) \subset D \in \mathcal{D}$ where level $(D)=k \in\{0, \ldots, L\}$ and $\pi\left(\hat{J}_{\varepsilon_{0}}(z)\right) \subset\left(z-\varepsilon_{0}, z+\right.$ $\left.\varepsilon_{0}\right)$. By 4.6), $\left(\hat{f}_{z, \varepsilon_{0}}^{j}\left(\hat{J}_{\varepsilon_{0}}(z)\right)\right) \cap \hat{I}_{z, \varepsilon_{0}}^{\prime}(L)=\emptyset$, for all $j=0, \ldots, L-k$. As a consequence $\pi^{-1}\left(\left(z-\varepsilon_{0}, z+\varepsilon_{0}\right)\right) \cap \hat{I}_{z, \varepsilon_{0}}^{\prime}(L)=\emptyset$ and there is a one-to-one correspondence between elements of $\hat{I}_{z, \varepsilon_{0}}^{\prime}(L)$ and $\hat{I}_{z, \varepsilon_{0}, \varepsilon}^{\prime}(L)$; indeed, precisely the same domains appear on each level. Abusing notation slightly, we write $\hat{I}_{z, \varepsilon_{0}}^{\prime}(L)=\hat{I}_{z, \varepsilon_{0}, \varepsilon}^{\prime}(L)$, and once $L$ is fixed, simply refer to the 
common set of domains as

$$
Y=\sqcup_{Q \in \hat{\mathcal{P}}_{L}\left(\mathrm{Crit}_{z, \varepsilon_{0}}\right)} Q .
$$

As a result of this construction, $Y \cap \hat{H}_{\varepsilon}=\emptyset$ for all $\varepsilon<\varepsilon_{0}$, where $\hat{H}_{\varepsilon}=\pi^{-1}\left(H_{\varepsilon}\right)$.

Remark 4.9. (Role of $\varepsilon$ and $\varepsilon_{0}$.) The cuts at $z \pm \varepsilon$ form the boundary of the hole $H_{\varepsilon}$, and defining $\hat{I}_{z, \varepsilon_{0}, \varepsilon}$ with respect to these cuts guarantees that the Markov structure will respect the hole. The extra cuts at $z \pm \varepsilon_{0}$ are used to guarantee uniform images and tails for returns to $Y$ as $\varepsilon \rightarrow 0$. Without loss of generality on $H_{\varepsilon}$, we may always choose $\varepsilon_{0}$ to satisfy,

$$
\left\{f^{\ell}\left(z \pm \varepsilon_{0}\right)\right\}_{\ell \geqslant 0} \cap \mathrm{Crit}_{z}=\emptyset \text { and }\left\{f^{\ell}(z)\right\}_{\ell \geqslant 0} \cap\left\{z \pm \varepsilon_{0}\right\}=\emptyset .
$$

In fact, we will only need to invoke (4.7) in Section 7 to prove convergence to the asymptotic escape rate in the case that $z$ is periodic (see Lemmas 7.6 and 7.11 ). All results in Sections $[4.5,55$ and 6 hold for all $\varepsilon_{0} \leqslant \varepsilon_{0}^{*}$.

The size of $\varepsilon>0$ will be further reduced in Corollaries 4.13 and 5.3, and Lemma 6.2 to satisfy $\varepsilon<\varepsilon_{1}^{*}$, where $\varepsilon_{1}^{*}<\varepsilon_{0}$ guarantees that the corresponding induced maps are uniformly mixing and the associated transfer operators have a uniform spectral gap.

As defined above, $\mathcal{Q}$ denotes the finite partition of $Y$ into its domains. Define the induced maps $F_{z, \varepsilon_{0}}=\hat{f}_{z, \varepsilon_{0}}^{R_{z, \varepsilon_{0}}}$ and $F_{z, \varepsilon_{0}, \varepsilon}=\hat{f}_{z, \varepsilon_{0}, \varepsilon}^{R_{z, \varepsilon}, \varepsilon}$ acting on the domain $Y$, where $R_{\varkappa}$ denotes the first return time to $Y$ in the extension $\left(\hat{f}_{\varkappa}, \hat{I}_{\varkappa}\right)$, and $\varkappa$ stands for either of the indices $\left(z, \varepsilon_{0}\right)$ or $\left(z, \varepsilon_{0}, \varepsilon\right)$. By construction, all images of elements of $\mathcal{Q}$ under $F_{\varkappa}$ are unions of elements of $\mathcal{Q}$. Thus $F_{\varkappa}$ has the finite images property.

We have a natural projection $\pi_{\varkappa}: \hat{I}_{\varkappa} \rightarrow I$ which commutes with the dynamics, $\pi_{\varkappa} \circ \hat{f}_{\varkappa}=f \circ \pi_{\varkappa}$. Note that from here on we will fix $\hat{m}=\hat{m}_{\varphi}$ for the relevant $\varphi=\phi-P(\phi)$. As in Remark 4.8, $\hat{m}$ is the same for all $Y=\hat{I}_{\varkappa}^{\prime}(L)$, and moreover $\hat{m}$ is always conformal for $\Phi_{\varkappa}$ under $F_{\varkappa}$ and we obtain $F_{\varkappa}$-invariant measures as in (4.4) and 4.5).

Define $\mathfrak{d}=\# \mathcal{C} r+5 \operatorname{deg}(f) \geqslant \# \operatorname{Crit}_{z, \varepsilon_{0}, \varepsilon}$, and note that by definition, $\mathfrak{d}$ does not depend on $\varepsilon$ and $\varepsilon_{0}$, just on the fact that we have introduced extra cuts at the preimages of the 5 points, $z, z \pm \varepsilon_{0}$ and $z \pm \varepsilon$. Our first result provides uniform bounds on the tail of the return time functions $R_{z, \varepsilon_{0}}$ and $R_{z, \varepsilon_{0}, \varepsilon}$.

Theorem 4.10. Suppose that either:

(a) $\phi=-t \log |D f|$ for $t \in\left(t^{-}, t^{+}\right)$; or

(b) $\phi: I \rightarrow \mathbb{R}$ is Hölder continuous.

Then there exist $L \in \mathbb{N}, C>0$ and $\alpha>0$ such that for all $0<\varepsilon<\varepsilon_{0} \leqslant \varepsilon_{0}^{*}$, $F_{\varkappa}$, the first return map to $\hat{I}_{\varkappa}^{\prime}(L)$, has tails $\hat{m}_{\phi-P(\phi)}\left(R_{\varkappa}>n\right) \leqslant C e^{-\alpha n}$ where $\varkappa=\left\{z, \varepsilon_{0}\right\}$ or $\left\{z, \varepsilon_{0}, \varepsilon\right\}$. Here $L, C, \alpha$ depend only on $(f, \phi, \mathfrak{d})$.

Proof of Theorem 4.10. For ease of notation, we will drop the subscript $\varkappa$ in the proof, but all statements apply equally well to $F_{z, \varepsilon_{0}}$ and $F_{z, \varepsilon_{0}, \varepsilon}$.

As shown in [DoT, Lemma 4.15], for each $\xi>0$ there exist $L=L(\xi)$ and $n_{0}=n_{0}(L)$ such that $\#\left\{i: R\left(Y_{i}\right)=n\right\} \leqslant e^{\xi n}$ for all $n \geqslant n_{0}$. Crucially these numbers only depend on $\mathfrak{d}$, so are independent of the actual values of $\varepsilon$ and $\varepsilon_{0}$. Thus to prove the theorem, it suffices to show that there exists some $\bar{\alpha}>\xi$ such that for any 1 -cylinder $Y_{i}$ of $F, \hat{m}\left(Y_{i}\right) \leqslant e^{-\bar{\alpha} R_{i}}$ where $R_{i}:=\left.R\right|_{Y_{i}}$. 
In the geometric case i.e., case (a), we will set

$$
\bar{\alpha}=p_{t}+t \lambda_{\min } .
$$

The fact that $\bar{\alpha}>0$ follows immediately from our having set $t<t^{+}$. In the Hölder case we obtain an analogous $\bar{\alpha}>0$ using the assumed pressure gap, $\phi<P(\phi)$. In both cases we now can select $\xi<\bar{\alpha}$, which then fixes $L(\xi)$ and $\mathcal{Q}$. We will see below that our estimates on the measures of the domains $Y_{i}$ yield $\alpha=\bar{\alpha}-\xi$.

We will use the expansion on periodic orbits to estimate the measure of the domains $Y_{i}$. The proof of this theorem would be simpler if we had $Y_{i} \subset F\left(Y_{i}\right)$ for all $i$, since then each $Y_{i}$ would contain a point of period $R_{i}$, which will allow us to connect $\bar{\alpha}$ and the measure of $Y_{i}$. To overcome this issue, we will first prove that $F$ is transitive on elements of $\mathcal{Q}$. Recall that by Lemma 4.2 , if $O_{1}, O_{2} \subset \hat{I}_{z, \varepsilon_{0}}^{\prime}(L)$ are two open sets such that $\pi\left(O_{1}\right) \cap \pi\left(O_{2}\right) \neq \emptyset$, then there exists $n \in \mathbb{N}$ such that $F^{n}\left(O_{1}\right) \cap F^{n}\left(O_{2}\right) \neq \emptyset$.

Now let $Q_{1}, Q_{2} \in \mathcal{Q}$. Since $f$ is leo, there exists $n_{1} \in \mathbb{N}$ such that $\pi\left(\hat{f}^{n_{1}} Q_{1}\right) \supset I \supset \pi\left(Q_{2}\right)$. By Lemma 4.2, there exists $n_{2} \in \mathbb{N}$ such that $\hat{f}^{n_{1}+n_{2}}\left(Q_{1}\right) \cap \hat{f}^{n_{2}}\left(Q_{2}\right) \neq \emptyset$. Since $Q_{2}$ is a recurrent element of $\mathcal{Q}$, there exists $n \in \mathbb{N}$ such that $F^{n}\left(Q_{1}\right) \cap Q_{2} \neq \emptyset$. Then the Markov property of $F$ implies that $F^{n}\left(Q_{1}\right) \supset Q_{2}$, and the claimed transitivity follows.

Since $\mathcal{Q}$ is finite, there exist $N \geqslant 1$ and $C>0$ such that for each pair $Q_{1}, Q_{2} \in \mathcal{Q}$, there are $J \subset Q_{1}$ and $n \leqslant N$ such that $\hat{f}^{n}: J \rightarrow Q_{2}$ is a diffeomorphism with $\left|D \hat{f}^{n}\right|_{J} \mid \geqslant C$. Therefore, each domain $Y_{i}$ of the inducing scheme contains a periodic point $y_{i}$ with period $R_{i} \leqslant p \leqslant R_{i}+N$ for $\hat{f}$. Then $\left|D F\left(y_{i}\right)\right| \geqslant C^{-1}\left|D \hat{f}^{p}\left(y_{i}\right)\right| \gtrsim e^{\lambda_{\min } R_{i}}$. Throughout we will treat $\hat{f}^{R_{i}}\left(Y_{i}\right)$ as having uniform size, i.e., independent of $i$.

In case (a), Lemma 4.1 implies

$$
\hat{m}\left(\hat{f}^{R_{i}}\left(Y_{i}\right)\right)=\int_{Y_{i}} e^{-S_{R_{i}}\left(t \hat{\phi}-p_{t}\right)} d \hat{m}=\int_{Y_{i}}|D F|^{t} e^{R_{i} p_{t}} d \hat{m} \asymp\left|D F\left(y_{i}\right)\right|^{t} \hat{m}\left(Y_{i}\right) e^{R_{i} p_{t}} .
$$

Therefore, $\hat{m}\left(Y_{i}\right) \lesssim e^{-R_{i}\left(t \lambda_{\min }+p_{t}\right)}=e^{-\bar{\alpha} R_{i}}$.

For the Hölder case, recall that we have assumed that $\phi<P(\phi)$, and thus by Remark $4.3, \hat{\phi}<P(\hat{\phi})$ on $\hat{I}$. Our value of $\bar{\alpha}$ here is $\inf \left\{P(\phi)-\frac{S_{p} \phi(x)}{p}: f^{p}(x)=x\right\} \geqslant \inf \{P(\phi)-\phi(x): x \in I\}>0$. So again, using a slightly more elementary version of the estimate in (4.8) in conjunction with Lemma 4.6, to give us our requisite distortion property, the result follows.

4.5. Uniform mixing for $F_{z, \varepsilon_{0}, \varepsilon}$. Now we choose $L$ large enough so that the conclusion of Theorem 4.10 is satisfied. Furthermore, we enlarge $L$ if necessary so that

a) $\kappa=\max \left\{\hat{m}\left(\hat{I}_{z, \varepsilon_{0}, \varepsilon} \backslash \hat{I}_{z, \varepsilon_{0}}^{\prime}(L)\right), \hat{m}\left(\hat{I}_{z, \varepsilon_{0}} \backslash \hat{I}_{z, \varepsilon_{0}}^{\prime}(L)\right)\right\}<1 / 3$; and

b) any ergodic invariant measure $\nu$ with entropy $h_{\nu}(f)>\left(\log \bar{\lambda}_{\varepsilon^{*}}+\alpha\right) / 2$ lifts to our inducing scheme on $\hat{I}_{z, \varepsilon_{0}}^{\prime}(L)$, where $\varepsilon^{*}$ is from Theorem 3.1 .

Item (b) is possible due to [DoT, Lemma 8.2], and the fact that $\alpha$ does not decrease as $L$ increases.

With $L$ fixed, we define $\varepsilon_{0}^{*}$ as in (4.6), and for $\varepsilon_{0} \leqslant \varepsilon_{0}^{*}$, we let $Y=\hat{I}_{z, \varepsilon_{0}}^{\prime}(L)$ as in Remark 4.8 .

Our next result proves a necessary mixing property for our return maps.

Lemma 4.11. For all $\varepsilon_{0} \leqslant \varepsilon_{0}^{*}$ and $\varepsilon<\varepsilon_{0}$, the induced maps $F_{z, \varepsilon_{0}}$ and $F_{z, \varepsilon_{0}, \varepsilon}$ are topologically mixing on $Y$. 
Proof. We write our arguments for $F_{z, \varepsilon_{0}, \varepsilon}$, but the same proof holds for $F_{z, \varepsilon_{0}}$.

By the proof of Theorem $4.10, F_{z, \varepsilon_{0}, \varepsilon}$ is transitive on the finitely many elements of $\mathcal{Q}$. The only way it can fail to be mixing is if the images decompose into a periodic cycle. Let $Q \in \mathcal{Q}$. Since $f$ is leo, there exists $n_{Q}$ such that $f^{n}(\pi(Q)) \supset I$ for all $n \geqslant n_{Q}$. By our choice of $L, \kappa=\hat{m}\left(\hat{I}_{z, \varepsilon_{0}, \varepsilon} \backslash Y\right)<1 / 3$. Then since $f^{n} \circ \pi=\pi \circ \hat{f}_{z, \varepsilon_{0}, \varepsilon}^{n}$, we have $m\left(\pi\left(\hat{f}_{z, \varepsilon_{0}, \varepsilon}^{n}(Q) \cap Y\right)\right) \geqslant 1-\kappa$, for $n \geqslant n_{Q}$.

Applying this to $n=n_{Q}$ and $n=n_{Q}+1$, we conclude

$$
m\left(\pi\left(Y \cap \hat{f}_{z, \varepsilon_{0}, \varepsilon}^{n_{Q}}(Q)\right) \cap \pi\left(Y \cap \hat{f}_{z, \varepsilon_{0}, \varepsilon}^{n_{Q}+1}(Q)\right)\right) \geqslant 1-2 \kappa>0 .
$$

Thus there must exist intervals $O_{1} \subset Y \cap \hat{f}_{z, \varepsilon_{0}, \varepsilon}^{n_{Q}}(Q)$ and $O_{2} \subset Y \cap \hat{f}_{z, \varepsilon_{0}, \varepsilon}^{n_{Q}+1}(Q)$ such that $\pi\left(O_{1}\right) \cap$ $\pi\left(O_{2}\right) \neq \emptyset$. By Lemma 4.2 , there exists $n_{1} \in \mathbb{N}$ such that $F_{z, \varepsilon_{0}, \varepsilon}^{n_{1}}\left(O_{1}\right) \cap F_{z, \varepsilon_{0}, \varepsilon}^{n_{1}}\left(O_{2}\right) \neq \emptyset$. Since $Q, O_{1}, O_{2} \subset Y$, there exists $k_{Q} \in \mathbb{N}$ such that $F_{z, \varepsilon_{0}, \varepsilon}^{k_{Q}}(Q) \cap F_{z, \varepsilon_{0}, \varepsilon}^{k_{Q}+1}(Q) \neq \emptyset$, so the period of $Q$ under $F_{z, \varepsilon_{0}, \varepsilon}$ is 1 . Thus $F_{z, \varepsilon_{0}, \varepsilon}$ is aperiodic and therefore mixing.

Our next two lemmas show that the mixing established in Lemma 4.11 is in fact uniform in $\varepsilon$.

Lemma 4.12. Fix $\varepsilon_{0} \leqslant \varepsilon_{0}^{*}$ and suppose there exist $Q_{1}, Q_{2} \in \mathcal{Q}$ and an interval $J \subset Q_{1}$ such that $F_{z, \varepsilon_{0}}^{n_{J}}(J)=Q_{2}$ for some $n_{J} \in \mathbb{N}$. Then there exists $\varepsilon_{1}<\varepsilon_{0}$ such that for all $\varepsilon \in\left(0, \varepsilon_{1}\right)$, $F_{z, \varepsilon_{0}, \varepsilon}^{n_{J}}(J)=Q_{2}$.

Proof. Fix $\varepsilon_{0}$. Suppose there exist $Q_{1}, Q_{2} \in \mathcal{Q}$ and an interval $J \subset Q_{1}$ and $n_{J} \in \mathbb{N}$ such that $F_{z, \varepsilon_{0}}^{n_{J}}(J)=Q_{2}$ as in the statement of the lemma. Let $n_{1} \in \mathbb{N}$ be such that $\hat{f}_{z, \varepsilon_{0}}^{n_{1}}(J)=Q_{2}$.

A key property of our construction of $Y=\hat{I}_{z, \varepsilon_{0}}^{\prime}(L)$ is that we have 'trimmed' the edges of the domains at returns: i.e., the endpoints of $Q_{1}$ and $Q_{2}$ are elements of $\partial \mathcal{P}_{L}=\partial \mathcal{P}_{L}\left(\mathrm{Crit}_{z, \varepsilon_{0}}\right)$ and the Markov property of $F_{z, \varepsilon_{0}}$, Lemma 4.4 implies that there exist domains $Q_{1}^{\prime} \supsetneq Q_{1}$ and $Q_{2}^{\prime} \supsetneq Q_{2}$ in the extension $\hat{I}_{z, \varepsilon_{0}}$ (note that $Q_{2}^{\prime}$ is an element of $\mathcal{D}$ ) and an interval $J^{\prime}$ with $J \subsetneq J^{\prime} \subset Q_{1}^{\prime}$ such that $\hat{f}_{z, \varepsilon_{0}}^{n_{1}}\left(J^{\prime}\right)=Q_{2}^{\prime}$.

Let $\widehat{z}$ and $\widehat{z \pm \varepsilon_{0}}$ denote the fibres above $z$ and $z \pm \varepsilon_{0}$, respectively. Due to the Markov property and because we have treated $f^{-1}(z)$ and $f^{-1}\left(z \pm \varepsilon_{0}\right)$ as cut points during our construction of $\hat{I}_{z, \varepsilon_{0}}$ and $\hat{I}_{z, \varepsilon_{0}, \varepsilon}$, it follows that $\partial\left(\hat{f}_{z, \varepsilon_{0}}^{j}(J)\right) \cap\left\{\widehat{z}, \widehat{z \pm \varepsilon_{0}}\right\}=\emptyset$, for all $0 \leqslant j \leqslant n_{1}$.

Case 1: $\bigcup_{j=0}^{n_{1}} \hat{f}_{z, \varepsilon_{0}}^{j}(J) \cap\left(\widehat{z-\varepsilon_{0}}, \widehat{z+\varepsilon_{0}}\right)=\emptyset$. Then introducing new cuts at $f^{-1}(z \pm \varepsilon)$ in the construction of $\hat{I}_{z, \varepsilon_{0}, \varepsilon}$ does not affect the endpoints of either $J^{\prime}$ or $Q_{2}^{\prime}$, and the lemma holds with $\varepsilon_{1}=\varepsilon_{0}$.

Case $2: \bigcup_{j=0}^{n_{1}} \hat{f}_{z, \varepsilon_{0}}^{j}(J) \cap\left(\widehat{z-\varepsilon_{0}}, \widehat{z+\varepsilon_{0}}\right) \neq \emptyset$. Choose

$$
\varepsilon_{1}<\min \left\{d\left(\partial\left(\hat{f}_{z, \varepsilon_{0}}^{j}(J)\right), \widehat{z}\right): 0 \leqslant j \leqslant n_{1}\right\} .
$$

It follows that for all $\varepsilon<\varepsilon_{1}, \hat{f}_{z, \varepsilon_{0}, \varepsilon}^{n_{1}}(J)=Q_{2}$. Moreover, there exists an interval $J_{\varepsilon}^{\prime} \supsetneq J$ and a domain $Q_{2, \varepsilon}^{\prime} \supsetneq Q_{2}$ in $\hat{I}_{z, \varepsilon_{0}, \varepsilon}$ such that $\hat{f}_{z, \varepsilon_{0}, \varepsilon}^{n_{1}}\left(J_{\varepsilon}^{\prime}\right)=Q_{2, \varepsilon}^{\prime}$. Then, since $F_{z, \varepsilon_{0}, \varepsilon}$ is the first return map to $Y$, and $Y$ is independent of $\varepsilon$, it follows that $F_{z, \varepsilon_{0}, \varepsilon}^{n_{J}}(J)=Q_{2}$.

Corollary 4.13. For all $\delta>0$ there exists $\varepsilon_{1} \in\left(0, \varepsilon_{0}\right)$ such that for all $\varepsilon \in\left(0, \varepsilon_{1}\right)$,

$$
\hat{m}\left(\hat{x} \in Y: F_{z, \varepsilon_{0}}(\hat{x}) \neq F_{z, \varepsilon_{0}, \varepsilon}(\hat{x})\right) \leqslant \delta .
$$


Proof. Fix $\delta>0$. By Theorem 4.10, we may choose $N$ such that $\hat{m}\left(R_{z, \varepsilon_{0}}>N\right) \leqslant C e^{-\alpha N} \leqslant \delta$. Considering the 1-cylinders for $F_{z, \varepsilon_{0}}$, there are only finitely many with $R \leqslant N$.

For each 1-cylinder $Y_{i}$, Lemma 4.12 yields an $\varepsilon_{1}(i)>0$ such that for all $\varepsilon \in\left(0, \varepsilon_{1}(i)\right)$, $Y_{i}$ is also a 1-cylinder for $F_{z, \varepsilon_{0}, \varepsilon}$; moreover, $F_{z, \varepsilon_{0}}\left(Y_{i}\right)=F_{z, \varepsilon_{0}, \varepsilon}\left(Y_{i}\right)$ and $R_{z, \varepsilon_{0}}\left(Y_{i}\right)=R_{z, \varepsilon_{0}, \varepsilon}\left(Y_{i}\right)$.

Taking $\varepsilon_{1}=\min \left\{\varepsilon_{1}(i): R_{z, \varepsilon_{0}}\left(Y_{i}\right) \leqslant N\right\}>0$ completes the proof of the corollary.

\section{A SPECTRAL GAP FOR THE INDUCED PUNCTURED TRANSFER OPERATORS}

In this section, we work with the induced maps $F_{z, \varepsilon_{0}}$ and $F_{z, \varepsilon_{0}, \varepsilon}$ defined on the common domain $Y=\hat{I}_{z, \varepsilon_{0}}^{\prime}(L)$. Since $z$ and $\varepsilon_{0} \leqslant \varepsilon_{0}^{*}$ are fixed throughout this section, for brevity, we will denote these maps simply by $F_{\varepsilon}:=F_{z, \varepsilon_{0}, \varepsilon}$ and $F_{0}:=F_{z, \varepsilon_{0}}$. Related objects will also be denoted by the subscript $\varepsilon$ or 0 . One of the main points of this section is to show that certain key properties are uniform for $\varepsilon \in\left[0, \varepsilon_{0}\right)$, where $\varepsilon=0$ is understood to correspond to the map $F_{z, \varepsilon_{0}}$ whose Hofbauer extension is defined by introducing cuts only at $z$ and $z \pm \varepsilon_{0}$.

For $\varepsilon \in\left[0, \varepsilon_{0}\right)$, let $\mathcal{Y}_{\varepsilon}=\left\{Y_{i}\right\}_{i}$ denote the set of 1-cylinders for $F_{\varepsilon}$ on which $R_{\varepsilon}=R_{z, \varepsilon_{0}, \varepsilon}$ is constant. As before, denote by $\mathcal{Q}$ the finite partition of $Y$ into intervals which comprise the finite images of $\mathcal{Y}_{\varepsilon}$ under $F_{\varepsilon}$. It is important that $Y$ and $\mathcal{Q}$ are independent of $\varepsilon$. Indeed, the uniformity of $\mathcal{Q}$ and $L$ allows us to take the constants in (4.3) and Lemma 4.6 uniformly in $\varepsilon$. This is formalised in properties (GM2) and (GM3) below.

Let $\Phi_{\varepsilon}=S_{R_{\varepsilon}} \varphi$ be the induced version of $\varphi$ on $Y$. Note that as in, for example [DoT, Lemma 14.9], the fact that $\hat{\mu}_{\varepsilon}\left(\hat{I}^{\prime}(L)\right)>0$ guarantees that $P\left(\Phi_{\varepsilon}\right)=0$. Also, the conformal measure $m_{\varphi}$ lifted to $\hat{I}_{z, \varepsilon_{0}, \varepsilon}$, and denoted $\hat{m}_{\varphi, \varepsilon}$, depends on both $\varepsilon$ and $\varphi$. However, $\hat{m}_{\varphi, \varepsilon}$ restricted to $Y$ is independent of $\varepsilon$ since $Y$ is independent of $\varepsilon$. Since we will work exclusively in $Y$ in this section, we suppress the dependence on $\varphi$ and refer to this measure on $Y$ as simply $\hat{m}$. For each $\varepsilon \in\left[0, \varepsilon_{0}\right)$, it is a conformal measure for $F_{\varepsilon}$ with respect to the potential $\Phi_{\varepsilon}$.

The key properties of the Gibbs-Markov maps $F_{\varepsilon}, \varepsilon \in\left[0, \varepsilon_{0}\right)$, are as follows:

(GM1) $F_{\varepsilon}\left(Y_{i}\right) \in \mathcal{Q}$ for each $Y_{i} \in \mathcal{Y}_{\varepsilon}$;

(GM2) There exist $\sigma>1$ and $C_{e} \in(0,1]$ (an expansion constant) such that for all $n \in \mathbb{N}$, if $Y_{i}^{(n)}$ is an $n$-cylinder for $F_{\varepsilon}$ and $x, y \in Y_{i}^{(n)}$, then $d\left(F_{\varepsilon}^{n} x, F_{\varepsilon}^{n} y\right) \geqslant C_{e} \sigma^{n} d(x, y)$, where $d(\cdot, \cdot)$ is the distance on each interval in $\hat{I}$ induced by the Euclidean metric on $I$.

(GM3) There exists $C_{d}>0$ (a distortion constant) such that for all $n \in \mathbb{N}$, if $Y_{i}^{(n)}$ is an $n$-cylinder for $F_{\varepsilon}$ and $x, y \in Y_{i}^{(n)}$, then

$$
\left|e^{S_{n} \Phi_{\varepsilon}(x)-S_{n} \Phi_{\varepsilon}(y)}-1\right| \leqslant C_{d} d\left(F_{\varepsilon}^{n} x, F_{\varepsilon}^{n} y\right)^{\eta},
$$

for some $\eta>0$.

Note that (GM3) follows from Lemma 4.6, and that the constants in (GM2) and (GM3) are independent of $\varepsilon$ by construction of $Y$. Due to (GM3), conformality and large images,

$$
e^{S_{n} \Phi_{\varepsilon}(x)} \leqslant\left(1+C_{d}\right) \frac{\hat{m}\left(Y_{i}^{(n)}\right)}{\hat{m}\left(F_{\varepsilon}^{n}\left(Y_{i}^{(n)}\right)\right)} \leqslant \frac{1+C_{d}}{q} \hat{m}\left(Y_{i}^{(n)}\right), \quad \text { for all } x \in Y_{i}^{(n)},
$$

where $q:=\min _{Q \in \mathcal{Q}} \hat{m}(Q)>0$. 
Let $\mathcal{C}^{\eta}(\mathcal{Q})$ denote the set of Hölder continuous functions on elements of $\mathcal{Q}$, equipped with the norm,

$$
\|\psi\|_{\mathcal{C}^{\eta}}=\sup _{Q \in \mathcal{Q}}\left(|\psi|_{\mathcal{C}^{0}(Q)}+\sup _{x, y \in Q}|\psi(x)-\psi(y)| d(x, y)^{-\eta}\right)=: \sup _{Q \in \mathcal{Q}}\left(|\psi|_{\mathcal{C}^{0}(Q)}+H_{Q}^{\eta}(\psi)\right) .
$$

We define the transfer operator $\mathcal{L}_{\varepsilon}=\mathcal{L}_{\Phi_{\varepsilon}}$ acting on $L^{1}\left(\hat{m}_{\varepsilon}\right)$ by

$$
\mathcal{L}_{\varepsilon}^{n} \psi(x)=\sum_{y \in F_{\varepsilon}^{-n}(x)} \psi(y) e^{S_{n} \Phi_{\varepsilon}(y)}, \quad \text { for each } n \geqslant 1 .
$$

Analogously, define $\mathcal{L}_{0}$ to be the transfer operator corresponding to the map $F_{0}=F_{z, \varepsilon_{0}}$.

Given a hole $H_{\varepsilon}=(z-\varepsilon, z+\varepsilon), \varepsilon \in\left(0, \varepsilon_{0}\right)$, as in Remark 4.8, its lift $\hat{H}_{\varepsilon}$ is disjoint from $Y$ due to our choice of $\varepsilon_{0}^{*}$. We denote by $\hat{H}_{\varepsilon}^{\prime} \subset Y$ the pre-hole, the set of points in $Y$ which do not return to $Y$ before entering $\hat{H}_{\varepsilon}$. Due to our construction, $\hat{H}_{\varepsilon}^{\prime}$ is a (countable) union of 1-cylinders for $F_{\varepsilon}$,

$$
\hat{H}_{\varepsilon}^{\prime}=\left\{Y_{i} \in \mathcal{Y}_{\varepsilon}: \hat{f}_{\varepsilon}^{n}\left(Y_{i}\right) \subset \hat{H}_{\varepsilon} \text { for some } n<R_{\varepsilon}\left(Y_{i}\right)\right\} \text {. }
$$

We will treat $\hat{H}_{\varepsilon}^{\prime}$ as our effective hole for $F_{\varepsilon}$. Let $\stackrel{\circ}{Y}_{\varepsilon}=Y \backslash \hat{H}_{\varepsilon}^{\prime}$, and for $n \geqslant 0$, define

$$
\stackrel{\circ}{Y}_{\varepsilon}^{n}=\cap_{i=0}^{n} F_{\varepsilon}^{-i}\left(\stackrel{\circ}{Y}_{\varepsilon}\right)
$$

to be the set of points which do not enter $\hat{H}_{\varepsilon}^{\prime}$ in the first $n$ iterates of $F_{\varepsilon}$. The dynamics of the induced open system are defined by $\stackrel{\circ}{\varepsilon}_{\varepsilon}^{n}=\left.F_{\varepsilon}^{n}\right|_{Y_{\varepsilon}^{n-1}}$. Since $\hat{H}_{\varepsilon}^{\prime}$ is a union of 1-cylinders for $F_{\varepsilon}$, the punctured map $\stackrel{\circ}{F}_{\varepsilon}$ has the same finite image property: $\stackrel{\circ}{F}_{\varepsilon}\left(Y_{i}\right) \in \mathcal{Q}$ for each $Y_{i} \subset \stackrel{\circ}{Y}_{\varepsilon}$. The punctured transfer operator for the open system is defined for $n \geqslant 1$ by

$$
\dot{\mathcal{L}}_{\varepsilon}^{n} \psi(x)=\mathcal{L}_{\varepsilon}^{n}\left(\psi 1_{\dot{Y}_{\varepsilon}^{n-1}}\right)(x)=\sum_{y \in \dot{F}_{\varepsilon}^{-n}(x)} \psi(y) e^{S_{n} \Phi_{\varepsilon}(y)} .
$$

The punctured transfer operator is defined only for $\varepsilon>0$. There is no analogous object for $\mathcal{L}_{0}$.

5.1. Spectral properties of $\mathcal{L}_{\varepsilon}$. In this section we prove that for sufficiently small $\varepsilon$, all the operators $\mathcal{L}_{\varepsilon}$ have a uniform spectral gap.

Proposition 5.1. There exists $C>0$ such that for all $n \geqslant 0$,

$$
\begin{aligned}
\left\|\dot{\mathcal{L}}_{\varepsilon}^{n} \psi\right\|_{\mathcal{C}^{\eta}} & \leqslant C \sigma^{-\eta n}\|\psi\|_{\mathcal{C}^{\eta}} \hat{m}\left(\dot{Y}_{\varepsilon}^{n-1}\right)+C \int_{\dot{Y}_{\varepsilon}^{n-1}}|\psi| d \hat{m} \quad \forall \psi \in \mathcal{C}^{\eta}(\mathcal{Q}), \\
\left|\dot{\mathcal{L}}_{\varepsilon}^{n} \psi\right|_{L^{1}(\hat{m})} & \leqslant \int_{\dot{Y}_{\varepsilon}^{n-1}}|\psi| d \hat{m} \quad \forall \psi \in L^{1}(\hat{m}) .
\end{aligned}
$$

The analogous inequalities hold for $\mathcal{L}_{\varepsilon}^{n} \psi$ and $\mathcal{L}_{0}^{n} \psi$ with $\stackrel{\circ}{\varepsilon}_{\varepsilon}^{n-1}$ replaced by $Y$.

Proof. Due to the definition (5.2), $\int_{Y} \dot{\mathcal{L}}_{\varepsilon}^{n} \psi d \hat{m}=\int_{Y_{\varepsilon}^{n-1}} \psi d \hat{m}$, so that (5.4) is immediate. We focus on verifying $(5.3)$ for $\psi \in \mathcal{C}^{\eta}(\mathcal{Q})$.

First, we estimate the Hölder constant of $\mathcal{L}_{\varepsilon}^{n} \psi$. Let $Q \in \mathcal{Q}$ and $x, y \in Q$. For $n \geqslant 0$, notice that each $u \in \stackrel{\circ}{F}_{\varepsilon}^{-n}(x)$ has a (unique) corresponding $v \in \stackrel{\circ}{F}_{\varepsilon}^{-n}(y)$ lying in the same $n$-cylinder $Y_{i}^{(n)}(u)$ as $u$. Thus,

$$
\begin{aligned}
& \stackrel{\circ}{\mathcal{L}}_{\varepsilon}^{n} \psi(x)-\stackrel{\circ}{\mathcal{L}}_{\varepsilon}^{n} \psi(y)=\sum_{u \in \dot{F}_{\varepsilon}^{-n}(x)}(\psi(u)-\psi(v)) e^{S_{n} \Phi_{\varepsilon}(u)}+\sum_{v \in \dot{F}_{\varepsilon}^{-n}(y)} \psi(v)\left(e^{S_{n} \Phi_{\varepsilon}(u)}-e^{S_{n} \Phi_{\varepsilon}(v)}\right) \\
& \leqslant \sum_{u \in \dot{F}_{\varepsilon}^{-n}(x)} H^{\eta}(\psi) d(u, v)^{\eta} \frac{1+C_{d}}{q} \hat{m}\left(Y_{i}^{(n)}(u)\right)+\sum_{v \in \dot{F}_{\varepsilon}^{-n}(y)}|\psi(v)| \hat{m}\left(Y_{i}^{(n)}(v)\right) \frac{1+C_{d}}{q} C_{d} d(x, y)^{\eta},
\end{aligned}
$$


where we have used the bounded distortion property (GM3) as well as (5.1). Now using the regularity of $\psi$ as well as the expanding property (GM2), for any $v \in \stackrel{\circ}{F}_{\varepsilon}^{-n}(y)$,

$$
|| \psi(v)\left|-\frac{1}{\hat{m}\left(Y_{i}^{(n)}(v)\right)} \int_{Y_{i}^{(n)}(v)}\right| \psi|d \hat{m}| \leqslant H^{\eta}(\psi) \operatorname{diam}\left(Y_{i}^{(n)}(v)\right)^{\eta} \leqslant H^{\eta}(\psi) C_{e}^{-\eta} \sigma^{-\eta n} .
$$

Putting these estimates together, we obtain

$$
\begin{aligned}
\left|\dot{\mathcal{L}}_{\varepsilon}^{n} \psi(x)-\dot{\mathcal{L}}_{\varepsilon}^{n} \psi(y)\right| \leqslant & C_{e}^{-\eta} \sigma^{-\eta n} H^{\eta}(\psi) d(x, y)^{\eta} \sum_{u \in \dot{F}_{\varepsilon}^{-n}(x)} \frac{1+C_{d}}{q}\left(1+C_{d}\right) \hat{m}\left(Y_{i}^{(n)}(u)\right) \\
& +\sum_{v \in \dot{F}_{\varepsilon}^{-n}(y)} \int_{Y_{i}^{(n)}(v)}|\psi| d \hat{m} \frac{1+C_{d}}{q} C_{d} d(x, y)^{\eta} .
\end{aligned}
$$

Due to the fact that the hole respects the Markov structure of our inducing scheme, it follows that $\cup_{u \in \dot{F}_{\varepsilon}^{-n}(x)} Y_{i}^{(n)}(u) \subset \stackrel{\circ}{Y}_{\varepsilon}^{n-1}$ allowing us to evaluate both sums. Now dividing through by $d(x, y)^{\eta}$ and taking the appropriate suprema yields the required inequality in $(5.3)$ for the Hölder constant of $\stackrel{\circ}{\mathcal{L}}_{\varepsilon}^{n} \psi$ with $C=C_{e}^{-\eta} \frac{\left(1+C_{d}\right)^{2}}{q}$.

Next, we estimate $\left|\dot{\mathcal{L}}_{\varepsilon}^{n} \psi\right|_{\infty}$. Let $Q \in \mathcal{Q}$ and $x \in Q$. Now,

$$
\left|\dot{\mathcal{L}}_{\varepsilon}^{n} \psi(x)\right| \leqslant \sum_{u \in \stackrel{\circ}{F}_{\varepsilon}^{-n}(x)}|\psi(u)| e^{S_{n} \Phi_{\varepsilon}(u)} \leqslant \sum_{u \in \stackrel{\circ}{\varepsilon}_{\varepsilon}^{-n}(x)} \frac{1+C_{d}}{q}|\psi(u)| \hat{m}\left(Y_{i}^{(n)}(u)\right)
$$

where we have used (5.1) for the second inequality. Using (5.5), we estimate,

$$
\begin{aligned}
\left|\dot{\mathcal{L}}_{\varepsilon}^{n} \psi(x)\right| & \leqslant \frac{1+C_{d}}{q} \sum_{u \in \dot{\circ}_{\varepsilon}^{-n}(x)} H^{\eta}(\psi) C_{e}^{-\eta} \sigma^{-\eta n} \hat{m}\left(Y_{i}^{(n)}(u)\right)+\int_{Y_{i}^{(n)}(u)}|\psi| d \hat{m} \\
& \leqslant C^{\prime} \sigma^{-\eta n} H^{\eta}(\psi) \hat{m}\left(\stackrel{\circ}{\varepsilon}_{\varepsilon}^{n-1}\right)+\int_{\dot{Y}_{\varepsilon}^{n-1}}|\psi| d \hat{m},
\end{aligned}
$$

so 5.3 holds with $C=2 C_{e}^{-\eta} \frac{\left(1+C_{d}\right)^{2}}{q}$, completing the proof of the proposition.

Define the norm for $\mathcal{L}_{\varepsilon}: \mathcal{C}^{\eta}(\mathcal{Q}) \rightarrow L^{1}(\hat{m})$ by

$$
\left\|\mathcal{L}_{\varepsilon}\right\| \mid=\sup \left\{\left|\mathcal{L}_{\varepsilon} \psi\right|_{L^{1}(\hat{m})}:\|\psi\|_{\mathcal{C}^{\eta}} \leqslant 1\right\} .
$$

Lemma 5.2. For any $\delta>0$, there exists $\varepsilon_{\delta}>0$ such that for all $\varepsilon \in\left(0, \varepsilon_{\delta}\right),\left\|\mathcal{L}_{0}-\mathcal{L}_{\varepsilon}\right\| \leqslant \delta$.

Proof. Fix $\delta>0$. Define $\mathcal{G}_{\varepsilon}=\left\{Y_{i} \in \mathcal{Y}_{\varepsilon}: \hat{f}_{z, \varepsilon_{0}}^{k}\left(Y_{i}\right)=\hat{f}_{z, \varepsilon_{0}, \varepsilon}^{k}\left(Y_{i}\right), \forall k=1, \ldots, R_{\varepsilon}\left(Y_{i}\right)\right\}$. Note that if $Y_{i} \in \mathcal{G}_{\varepsilon}$, then $\Phi_{0}=\Phi_{\varepsilon}$ on $Y_{i}$.

Next define $B_{\varepsilon}=\left\{y \in Y: Y_{i, \varepsilon}(y) \notin \mathcal{G}_{\varepsilon}\right\}$, where $Y_{i, \varepsilon}(y)$ is the 1-cylinder with respect to $F_{\varepsilon}$ containing $y$. For $\psi \in \mathcal{C}^{\eta}(\mathcal{Q})$ and $x \in Y$,

$$
\begin{aligned}
\left|\left(\mathcal{L}_{0}-\mathcal{L}_{\varepsilon}\right) \psi(x)\right| & =\left|\sum_{y \in F_{0}^{-1} x} \psi(y) e^{\Phi_{0}(y)}-\sum_{y \in F_{\varepsilon}^{-1} x} \psi(y) e^{\Phi_{\varepsilon}(y)}\right| \\
& \leqslant|\psi|_{\infty} \sum_{\substack{y \in F_{0}^{-1} x \\
y \in B_{\varepsilon}}} \frac{1+C_{d}}{q} \hat{m}\left(Y_{i}(y)\right)+|\psi|_{\infty} \sum_{\substack{y \in F_{\varepsilon}^{-1} x \\
y \in B_{\varepsilon}}} \frac{1+C_{d}}{q} \hat{m}\left(Y_{i}(y)\right) .
\end{aligned}
$$


By the proof of Corollary 4.13 , the total mass of 1 -cylinders where $F_{0}$ and $F_{\varepsilon}$ do not agree can be made arbitrarily small.

Let $\delta^{\prime}=\delta \frac{q}{2\left(1+C_{d}\right) \hat{m}(Y)}$. Choose $\varepsilon_{\delta}>0$ such that $\hat{m}\left(B_{\varepsilon_{\delta}}\right) \leqslant \delta^{\prime}$ by Corollary 4.13 . Then

$$
\left|\left(\mathcal{L}_{0}-\mathcal{L}_{\varepsilon}\right) \psi(x)\right| \leqslant|\psi|_{\infty} 2 \frac{1+C_{d}}{q} \hat{m}\left(B_{\varepsilon}\right) \leqslant|\psi|_{\infty} \delta / \hat{m}(Y) .
$$

Integrating over $x \in Y$ proves the lemma: $\int\left|\left(\mathcal{L}_{0}-\mathcal{L}_{\varepsilon}\right) \psi\right| d \hat{m} \leqslant|\psi|_{\infty} \delta$.

Corollary 5.3. There exists $\varepsilon_{1} \in\left(0, \varepsilon_{0}\right]$ such that the family of operators $\mathcal{L}_{\varepsilon}, \varepsilon \in\left[0, \varepsilon_{1}\right)$, acting on $\mathcal{C}^{\eta}(\mathcal{Q})$ have a uniform spectral gap. There exists $\beta>0$ such that $\mathcal{L}_{\varepsilon}$ admits the following spectral decomposition for all $\varepsilon \in\left[0, \varepsilon_{1}\right)$ : There exist $G_{\varepsilon} \in \mathcal{C}^{\eta}(\mathcal{Q})$, a linear functional $e_{\varepsilon}: \mathcal{C}^{\eta}(\mathcal{Q}) \rightarrow \mathbb{R}$ and an operator $\mathcal{R}_{\varepsilon}: \mathcal{C}^{\eta}(\mathcal{Q}) \circlearrowleft$ such that

$$
\mathcal{L}_{\varepsilon}=G_{\varepsilon} \otimes e_{\varepsilon}+\mathcal{R}_{\varepsilon}, \quad \text { and } \mathcal{R}_{\varepsilon} G_{\varepsilon}=0 .
$$

The spectral radius of $\mathcal{R}_{\varepsilon}$ is at most $e^{-\beta}$ and $e_{\varepsilon}(\psi)=\int_{Y} \psi d \hat{m}$ for all $\psi \in \mathcal{C}^{\eta}(\mathcal{Q})$.

Moreover, $G_{\varepsilon} \rightarrow G_{0}$ in $L^{1}(\hat{m})$ and $\left\|\mathcal{R}_{\varepsilon}-\mathcal{R}_{0}\right\| \rightarrow 0$ as $\varepsilon \rightarrow 0$.

We may normalise the above so that $\hat{m}\left(G_{\varepsilon}\right)=1$, so $\hat{\mu}_{Y, \varepsilon}=G_{\varepsilon} \hat{m}$ is the corresponding invariant probability measure for $F_{\varepsilon}$.

Proof. The fact that all the operators $\mathcal{L}_{\varepsilon}, \varepsilon \in\left[0, \varepsilon_{0}\right)$, are quasi-compact on $\mathcal{C}^{\eta}(\mathcal{Q})$ with essential spectral radius bounded by $\sigma^{-1}$ follows from Proposition 5.1 and the fact that the unit ball of $\mathcal{C}^{\eta}(\mathcal{Q})$ is compactly embedded in $L^{1}(\hat{m})$. Moreover, the spectrum of $\mathcal{L}_{\varepsilon}$ on the unit circle is finite dimensional and forms a cyclic group.

Since $F_{0}$ is mixing by Lemma 4.11, $\mathcal{L}_{0}$ has a single simple eigenvalue at 1 and the rest of the spectrum of $\mathcal{L}_{0}$ is contained in a disk of radius $e^{-2 \beta} \geqslant \sigma^{-1}$ for some $\beta>0$. Next, by Lemma 5.2 and [KL1, Corollary 1], the spectrum of $\mathcal{L}_{\varepsilon}$ outside the disk of radius $\sigma^{-1}$ can be made arbitrarily close to that of $\mathcal{L}_{0}$ by choosing $\varepsilon$ sufficiently small. Thus we may choose $\varepsilon_{1} \in\left(0, \varepsilon_{0}\right]$ such that the spectrum of $\mathcal{L}_{\varepsilon}$ outside the disk of radius $e^{-\beta}$ consists only of a simple eigenvalue at 1 , for all $\varepsilon \in\left(0, \varepsilon_{1}\right)$. The closeness of $G_{\varepsilon}$ and $\mathcal{R}_{\varepsilon}$ to $G_{0}$ and $\mathcal{R}_{0}$ follow similarly from [KL1, Corollary 1]. Finally, the fact that $e_{\varepsilon}(\psi)=\hat{m}(\psi)$ for all $\psi \in \mathcal{C}^{\eta}(\mathcal{Q})$ follows from the conformality of $\hat{m}$.

5.2. Spectral properties of the punctured operators $\stackrel{\circ}{\mathcal{L}}_{\varepsilon}$. Due to the uniform Lasota-Yorke inequalities provided by Proposition 5.1 , it only remains to show that $\mathcal{L}_{\varepsilon}$ and $\dot{\mathcal{L}}_{\varepsilon}$ are close in the $\|\mid\|$ ||-norm.

Lemma 5.4. For any $\varepsilon \in\left(0, \varepsilon_{0}\right),\left\|\mathcal{L}_{\varepsilon}-\stackrel{\circ}{\mathcal{L}}_{\varepsilon}\right\| \leqslant \hat{m}\left(\hat{H}_{\varepsilon}^{\prime}\right)$.

Proof. The proof is immediate using the definition of $\stackrel{\circ}{\mathcal{L}}_{\varepsilon}$ and the conformality of $\hat{m}$,

$$
\int\left(\mathcal{L}_{\varepsilon}-\dot{\mathcal{L}}_{\varepsilon}\right) \psi d \hat{m}=\int \mathcal{L}_{\varepsilon}\left(1_{Y \backslash \dot{Y}_{\varepsilon}^{1}} \psi\right) d \hat{m}=\int_{\hat{H}_{\varepsilon}^{\prime}} \psi d \hat{m} \leqslant|\psi|_{\infty} \hat{m}\left(\hat{H}_{\varepsilon}^{\prime}\right),
$$

since $\hat{H}_{\varepsilon}^{\prime}=Y \backslash \stackrel{\circ}{Y}_{\varepsilon}$.

Corollary 5.5. There exists $\varepsilon_{2} \leqslant \varepsilon_{1}$ such that for all $\varepsilon \in\left(0, \varepsilon_{2}\right)$, the operators $\mathcal{L}_{\varepsilon}$ have a uniform spectral gap: There exist $\Lambda_{\varepsilon} \in\left(e^{-\beta / 3}, 1\right), \stackrel{\circ}{G}_{\varepsilon} \in \mathcal{C}^{\eta}(\mathcal{Q})$, a functional $\stackrel{\circ}{\varepsilon}_{\varepsilon}: \mathcal{C}^{\eta}(\mathcal{Q}) \rightarrow \mathbb{R}$, and an operator $\stackrel{\circ}{\mathcal{R}}_{\varepsilon}: \mathcal{C}^{\eta}(\mathcal{Q}) \circlearrowleft$ such that

$$
\stackrel{\circ}{\mathcal{L}}_{\varepsilon}=\Lambda_{\varepsilon} \stackrel{\circ}{G}_{\varepsilon} \otimes \stackrel{\circ}{e}_{\varepsilon}+\stackrel{\circ}{\mathcal{R}}_{\varepsilon}, \quad \text { and } \stackrel{\circ}{\mathcal{R}}_{\varepsilon} \stackrel{\circ}{G}_{\varepsilon}=0
$$


The spectral radius of $\dot{\mathcal{R}}_{\varepsilon}$ is at most $e^{-2 \beta / 3}<\Lambda_{\varepsilon}$.

Moreover, $\Lambda_{\varepsilon} \rightarrow 1, \stackrel{\circ}{G}_{\varepsilon} \rightarrow G_{0}$ in $L^{1}(\hat{m})$ and $\left\|\stackrel{\circ}{\mathcal{R}}_{\varepsilon}-\mathcal{R}_{0}\right\| \rightarrow 0$ as $\varepsilon \rightarrow 0$.

Proof. Lemmas 5.2 and 5.4 together with the triangle inequality show that $\stackrel{\circ}{\mathcal{L}}_{\varepsilon}$ and $\mathcal{L}_{0}$ are close in the $\|\cdot \mid\|$-norm. The uniform Lasota-Yorke inequalities given by Proposition 5.1 together with [KL1, Corollary 1] imply that the spectrum (and corresponding spectral projectors) of $\dot{\mathcal{L}}_{\varepsilon}$ outside the disk of radius $e^{-\beta}$ are close to those of $\mathcal{L}_{0}$. Without requiring a rate of approach, we may choose $\varepsilon_{2}>0$ with the stated properties.

We may normalise $\stackrel{\circ}{G}_{\varepsilon}$ and $\stackrel{\circ}{e}_{\varepsilon}$ so that $\hat{m}\left(\stackrel{\circ}{G}_{\varepsilon}\right)=1$ and $\stackrel{\circ}{e}_{\varepsilon}\left(\stackrel{\circ}{G}_{\varepsilon}\right)=1$, so that $\stackrel{\circ}{\mathcal{L}}_{\varepsilon} \stackrel{\circ}{G}_{\varepsilon}=\Lambda_{\varepsilon} \stackrel{\circ}{G}_{\varepsilon}$.

\section{Young towers AND PROOF of TheOREm 3.1}

The Markov structure of the return map $F_{\varepsilon}=F_{z, \varepsilon_{0}, \varepsilon}$ to $Y$ immediately implies the existence of another, related extension, called a Young tower. These have been well-studied in the context of open systems, so we will recall their structure in order to apply some results in our setting.

As in Section 5, let $R_{\varepsilon}=R_{z, \varepsilon_{0}, \varepsilon}$. Define the Young tower over $Y$ with return time $R_{\varepsilon}$ by,

$$
\Delta:=\left\{(y, \ell) \in Y \times \mathbb{N}: \ell<R_{\varepsilon}(y)\right\} .
$$

We view $\Delta$ as a tower with $\Delta_{\ell}=\{(y, n) \in Y \times \mathbb{N}: n=\ell\}$ as the $\ell$ th level. The dynamics on the tower is defined by $f_{\Delta}(y, \ell)=(y, \ell+1)$ when $\ell+1<R_{\varepsilon}(y)$, and $f_{\Delta}(y, \ell)=\left(F_{\varepsilon}(y), 0\right)$ otherwise. Thus $\Delta_{0}$ corresponds to $Y$ and $F_{\varepsilon}=f_{\Delta}^{R_{\varepsilon}}$ can be viewed as the first return map to $\Delta_{0}$. With this definition, there is a natural projection $\hat{\pi}_{\Delta}: \Delta \rightarrow \hat{I}$ satisfying $\hat{\pi}_{\Delta} \circ f_{\Delta}=\hat{f}_{\varepsilon} \circ \hat{\pi}_{\Delta}$. Then also defining $\pi_{\Delta}=\pi \circ \hat{\pi}_{\Delta}: \Delta \rightarrow I$, we have $\pi_{\Delta} \circ f_{\Delta}=f \circ \pi_{\Delta}$.

Clearly, $\Delta=\Delta\left(z, \varepsilon_{0}, \varepsilon\right)$ depends on $z, \varepsilon_{0}$ and $\varepsilon$ through the construction of $\hat{I}_{z, \varepsilon_{0}, \varepsilon}, Y=\hat{I}_{z, \varepsilon_{0}, \varepsilon}^{\prime}(L)$ and $F_{\varepsilon}$. However, since we fix these three parameters in this section, we will drop explicit mention of this dependence in the notation we use for objects associated with $\Delta$.

The map $f_{\Delta}$ inherits a Markov structure as follows. On $\Delta_{0}$, we use the elements of the finite partition $\mathcal{Q}$ as our partition elements, labelling them by $\Delta_{0, i}$. On $\Delta_{\ell}, \ell \geqslant 1$, we define $\Delta_{\ell, i}=f_{\Delta}^{\ell}\left(Y_{i}\right)$, $Y_{i} \in \mathcal{Y}_{\varepsilon}$. The collection $\left\{\Delta_{\ell, i}\right\}_{\ell, i \geqslant 0}$ forms a countable Markov partition for $f_{\Delta}$. Since at return times to $\Delta_{0}, f_{\Delta}$ maps the image of each 1-cylinder $Y_{i}$ to an element of the finite partition $\mathcal{Q}$ of $Y=\Delta_{0}$, we will view $\left(f_{\Delta}, \Delta\right)$ as a Young tower with finitely many bases. The partition $\left\{\Delta_{\ell, i}\right\}$ is generating since $\left\{Y_{i}\right\}_{i}$ is a generating partition for $F_{\varepsilon}$. Moreover, the first return time $R_{\varepsilon}$ to $\Delta_{0}$ under $f_{\Delta}$ is the same as the first return to $Y$ under $\hat{f}_{\varepsilon}$.

We make $\Delta$ into a metric space by defining a symbolic metric based on the Markov partition. Let $R_{\varepsilon}^{n}(x)$ denote the $n$th return time of $x$ to $\Delta_{0}$. Define the separation time on $\Delta_{0}$ by,

$$
s(x, y)=\min \left\{n \geqslant 0: f_{\Delta}^{R_{\varepsilon}^{n}}(x) \text { and } f_{\Delta}^{R_{\varepsilon}^{n}}(y) \text { lie in different elements of } \mathcal{Y}_{\varepsilon}\right\} .
$$

We extend the separation time to all of $\Delta$ by setting $s(x, y)=s\left(f_{\Delta}^{-\ell} x, f_{\Delta}^{-\ell} y\right)$ for $x, y \in \Delta_{\ell}$. It follows that $s(x, y)$ is finite almost everywhere since $\left\{\Delta_{i, j}\right\}$ is a generating partition. For $\theta>0$, define a metric on $\Delta$ by $d_{\theta}(x, y)=e^{-\theta s(x, y)}$. We will choose $\theta$ according to property (P3) in Section 6.1.

Given our (normalised) potential $\hat{\varphi}$ on $\hat{I}$, and $\hat{\varphi}$-conformal measure $\hat{m}=\hat{m}_{\varphi}$, we define a reference measure $m_{\Delta}$ on $\Delta$ by setting $m_{\Delta}=\hat{m}$ on $\Delta_{0}$, and $\left.m_{\Delta}\right|_{\Delta_{\ell}}:=\left(f_{\Delta}\right)_{*}\left(\left.m_{\Delta}\right|_{\Delta_{\ell-1} \cap f_{\Delta}^{-1} \Delta_{\ell}}\right)$. 
Similarly, we lift the potential $\hat{\varphi}$ to a potential $\varphi_{\Delta}$ on $\Delta$ as follows. For $x \in \Delta_{\ell}$, let $x^{-}=f_{\Delta}^{-\ell}(x)$ denote the pullback of $x$ to $\Delta_{0}$. Then,

$$
\varphi_{\Delta}(x):=S_{R_{\varepsilon}} \hat{\varphi}\left(x^{-}\right) \text {for } x \in f_{\Delta}^{-1}\left(\Delta_{0}\right) \text { and } \varphi_{\Delta}=0 \text { on } \Delta \backslash f_{\Delta}^{-1}\left(\Delta_{0}\right) .
$$

With this definition, $m_{\Delta}$ is a $\varphi_{\Delta}$-conformal measure.

We may also define a related invariant measure on $\Delta$. Let $G_{\varepsilon} \in \mathcal{C}^{\eta}(\mathcal{Q})$ be the invariant density from Corollary 5.3. Define

$$
g_{\Delta}=G_{\varepsilon} \text { on } \Delta_{0}, \text { and } g_{\Delta}(x)=G_{\varepsilon}\left(x^{-}\right) \text {for } x \in \Delta_{\ell}, \ell \geqslant 1,
$$

where $x^{-}$is defined as above.

It follows that the measure $d \mu_{\Delta}=g_{\Delta} d m_{\Delta} / \int_{\Delta} g_{\Delta} d m_{\Delta}$ is an invariant probability measure for $f_{\Delta}$. Moreover, we have $\left(\hat{\pi}_{\Delta}\right)_{*} \mu_{\Delta}=\hat{\mu}_{\varepsilon}$. And since $\pi_{*} \hat{\mu}_{\varepsilon}=\mu_{\varphi}$, we have also that $\left(\pi_{\Delta}\right)_{*} \mu_{\Delta}=\mu_{\varphi}$. Note that here $\hat{\mu}_{\varepsilon}$ is defined on $\hat{I}_{z, \varepsilon_{0}, \varepsilon}$ and depends on $\varepsilon$, while $\mu_{\varphi}$ does not.

We lift the hole $H=H(z, \varepsilon)$ to $\Delta$ by settting $H_{\Delta}:=\pi_{\Delta}^{-1} H=\hat{\pi}_{\Delta}^{-1} \hat{H}$. Due to the construction of $\hat{I}_{z, \varepsilon_{0}, \varepsilon}, H_{\Delta}$ comprises a countable collection of elements of the Markov partition $\Delta_{\ell, j}$, which we shall denote by $H_{\ell, j}$. Set $\stackrel{\circ}{\Delta}=\Delta \backslash H_{\Delta}$, and define the open system ${\stackrel{\circ}{f_{\Delta}}}=\left.f_{\Delta}\right|_{\stackrel{\Delta}{\Delta}}$.

Lemma 6.1. Define $\stackrel{\circ}{\Delta}^{(n)}=\cap_{i=0}^{n} f_{\Delta}^{-1}(\stackrel{\circ}{\Delta})$. Then,

$$
\log \bar{\lambda}_{\varepsilon}:=\limsup _{n \rightarrow \infty} \frac{1}{n} \log \mu_{\varphi}\left(\stackrel{\circ}{I}^{n}\right)=\limsup _{n \rightarrow \infty} \frac{1}{n} \log \mu_{\Delta}\left(\stackrel{\circ}{\Delta}^{(n)}\right)=\limsup _{n \rightarrow \infty} \frac{1}{n} \log m_{\Delta}\left(\stackrel{\circ}{\Delta}^{(n)}\right) .
$$

Proof. The first equality follows immediately from the fact that $\left(\pi_{\Delta}\right)_{*} \mu_{\Delta}=\mu_{\varphi}$ and $\pi_{\Delta} \circ f_{\Delta}=f \circ \pi_{\Delta}$, so that $\mu_{\Delta}\left(\AA^{(n)}\right)=\mu_{\varphi}\left(I^{n}\right)$ for each $n$. The second equality follows from the fact that $\mu_{\Delta}=g_{\Delta} m_{\Delta}$, and $g_{\Delta}$ is bounded (uniformly in $\varepsilon$ ) away from 0 and $\infty$ on $\Delta$ by (6.1) and Lemma 7.1 below.

Our final lemma of this subsection says that the open system $f_{\Delta}$ is mixing ${ }^{4}$ on partition elements under our assumptions on $f$ and our construction of $\hat{f}_{z, \varepsilon_{0}, \varepsilon}$.

Let $\varepsilon_{1}^{*}=\min \left\{\varepsilon_{1}\left(Q_{1}, Q_{2}\right): Q_{1}, Q_{2} \in \mathcal{Q}\right\}>0$, where $\varepsilon_{1}\left(Q_{1}, Q_{2}\right)$ is from Lemma 4.12 .

Lemma 6.2. For all $\varepsilon \in\left(0, \varepsilon_{1}^{*}\right)$, the open system $\left(f_{\Delta}^{\circ}, \stackrel{\circ}{\Delta}\right)$ is transitive and aperiodic on elements of $\left\{\Delta_{\ell, j}\right\}$ that do not lie above a component of $H_{\Delta}$.

Proof. Transitivity of $f_{\Delta}$ on elements of the Markov partition is guaranteed by the transitivity of $F_{z, \varepsilon_{0}, \varepsilon}$, proved in Lemma 4.11 . That this property carries over to the open map $f_{\Delta}$ follows from Lemma 4.12. Considering Case 2 in the proof of that lemma, we see that for $\varepsilon \in\left(0, \varepsilon_{1}\right)$, the orbit of the desired interval $J$ connecting $Q_{1}$ to $Q_{2}$ is disjoint from $H_{\varepsilon}$. Thus the connection holds for the open system $\left(f_{\Delta}, \stackrel{\circ}{\Delta}\right)$.

Next we show that $f_{\Delta}$ is aperiodic. Due to the structure of the tower map, it suffices to show that there exists $n_{0} \in \mathbb{N}$ such that for all $n \geqslant n_{0}, f_{\Delta}^{n}\left(\Delta_{0}\right) \supset \Delta_{0}$. Since returns to $\Delta_{0}$ must be to one of the finitely many elements of the partition $\mathcal{Q}$, this property is in turn implied by the following claim: For all $Q \in \mathcal{Q}$, there exists $n_{Q} \in \mathbb{N}$ such that $f_{\Delta}^{n_{Q}}(Q) \supset Q$ and ${\stackrel{\circ}{f_{Q}}}^{n_{Q}+1}(Q) \supset Q$. We proceed to prove the claim, which is a refinement of the proof of Lemma 4.11 .

\footnotetext{
${ }^{4}$ Mixing for an open system is not generally defined, and topologically transitivity does not hold unless we restrict to the survivor set $\check{\Delta}^{(\infty)}=\cap_{n=0}^{\infty} \stackrel{\Delta}{ }^{(n)}$. In the open systems context, a mixing property can be formulated in terms of transitions between elements of the Markov partition $\left\{\Delta_{\ell, j}\right\}$, after removing those elements which lie above components of $H_{\Delta}$ in $\Delta$.
} 
Let $Q \in \mathcal{Q}$. Since $f$ is leo, there exists $\bar{n} \in \mathbb{N}$ such that $f^{n}(\pi(Q)) \supset I$ for all $n \geqslant \bar{n}$. Thus as in the proof of Lemma 4.11, $m_{\varphi}\left(\pi_{\Delta}\left(f_{\Delta}^{n}(Q) \cap \Delta_{0}\right)\right) \geqslant 1-\kappa$, by choice of $L$. Applying this to $n=\bar{n}$ and $n=\bar{n}+1$, and recalling that we identify $Y=\hat{I}_{z, \varepsilon_{0}}^{\prime}$ with $\Delta_{0}$, we obtain,

$$
m_{\varphi}\left(\pi_{\Delta}\left(\Delta_{0} \cap f_{\Delta}^{\bar{n}}(Q)\right) \cap \pi_{\Delta}\left(\Delta_{0} \cap f_{\Delta}^{\bar{n}+1}(Q)\right)\right) \geqslant 1-2 \kappa>0 .
$$

Thus there must exist intervals $O_{1} \subset \Delta_{0} \cap f_{\Delta}^{\bar{n}}(Q)$ and $O_{2} \subset \Delta_{0} \cap f_{\Delta}^{\bar{n}+1}(Q)$ such that $\pi\left(O_{1}\right) \cap \pi\left(O_{2}\right) \neq$ $\emptyset$. By Lemma 4.2 , there exists $n_{1} \in \mathbb{N}$ such that $\hat{f}_{\varepsilon}^{n_{1}}\left(O_{1}\right) \cap \hat{f}_{\varepsilon}^{n_{1}}\left(O_{2}\right) \neq \emptyset$, and we can choose this time $n_{1}$ so that this intersection occurs in $Y=\Delta_{0}$. This implies that also $f_{\Delta}^{n_{1}}\left(O_{1}\right) \cap f_{\Delta}^{n_{1}}\left(O_{2}\right) \neq \emptyset$.

Now using the transitivity of $F_{\varepsilon}$, as well as its Markov property, there exists $k \in \mathbb{N}$ such that $F_{\varepsilon}^{k}\left(f_{\Delta}^{n_{1}}\left(O_{1}\right) \cap f_{\Delta}^{n_{1}}\left(O_{2}\right)\right) \supset Q$. Let $r_{k}$ denote the number of iterates of $f_{\Delta}$ contained in $F_{\varepsilon}^{k}$ on this set. This implies that both $f_{\Delta}^{r_{k}+n_{1}+\bar{n}}(Q) \supset Q$, and $f_{\Delta}^{r_{k}+n_{1}+\bar{n}+1}(Q) \supset Q$.

As a final step, we invoke Lemma 4.12 as earlier. We have constructed two times $k_{1}$ and $k_{2}$ for which $F_{\varepsilon}^{k_{j}}(Q) \supset Q, j=1,2$. By case 2 of the proof of Lemma 4.12 , for $\varepsilon<\varepsilon_{1}(Q, Q)$, these connections still occur in the open system. Thus we conclude that both $f_{\Delta}^{r_{k}+n_{1}+\bar{n}}(Q) \supset Q$, and $f_{\Delta}^{r_{k}+n_{1}+\bar{n}+1}(Q) \supset Q$, as required.

6.1. Transfer Operator on $\Delta$ and a Spectral Gap. In order to study the dynamics on the open tower, we define the transfer operator associated with the potential $\varphi_{\Delta}$,

$$
\mathcal{L}_{\Delta} \psi=\sum_{y \in f_{\Delta}^{-1} x} \psi(y) e^{\varphi_{\Delta}(y)},
$$

and its usual punctured counterpart for the open system, $\stackrel{\circ}{\mathcal{L}}_{\Delta} \psi=\mathcal{L}_{\Delta}\left(\psi \cdot 1_{\check{\triangle}^{(1)}}\right)$. We also define the corresponding punctured potential on the tower by $\varphi_{\Delta}^{H_{\varepsilon}}=\varphi_{\Delta}$ on $\stackrel{\circ}{\Delta}$ and $\varphi_{\Delta}^{H_{\varepsilon}}=-\infty$ on $H_{\Delta}$.

We will prove that for sufficiently small holes $H_{\varepsilon}$, the transfer operator $\dot{\mathcal{L}}_{\Delta}$ has a spectral gap on a certain Banach space $\mathcal{B}$, using the abstract result [DT2, Theorem 4.12]. Note that this result is not perturbative, but rather relies on checking four explicit conditions (P1)-(P4) from [DT2, Section 4.2]. They are as follows.

(P1) Exponential Tails. This follows from Theorem 4.10, since by definition of $m_{\Delta}$,

$$
m_{\Delta}\left(\Delta_{n}\right)=m_{\Delta}\left(\Delta_{0} \cap\left\{R_{\varepsilon}>n\right\}\right)=\hat{m}_{\varphi}\left(Y \cap\left\{R_{\varepsilon}>n\right\}\right) \leqslant C e^{-\alpha n},
$$

where $C$ and $\alpha$ are uniform for $\varepsilon<\varepsilon_{0}$.

(P2) Slow Escape. $\quad-\log \bar{\lambda}_{\varepsilon}<\alpha$. This can be guaranteed by noting that $\bar{\lambda}_{\varepsilon} \geqslant \Lambda_{\varepsilon}$, where $\Lambda_{\varepsilon}<1$ is from Corollary 5.5. This inequality is due to the fact that the escape from the induced system cannot be slower than the escape from the uninduced system. The requirement on the upper escape rate in [DT2] is defined in terms of $m_{\Delta}$, which in our case is equal to $\log \bar{\lambda}_{\varepsilon}$ by Lemma 6.1. Again using Corollary 5.5, there exists $\varepsilon^{*}>0$ such that $\Lambda_{\varepsilon}>e^{-\alpha}$ for all $\varepsilon \in\left(0, \varepsilon^{*}\right)$. This guarantees (P2).

(P3) Bounded Distortion and Lipschitz Property for $e^{\varphi_{\Delta}}$. The potential $\varphi_{\Delta}=0$ on $\Delta \backslash f_{\Delta}^{-1}\left(\Delta_{0}\right)$ so we need only to verify this property at return times. This follows from Lemma 4.6 and the following estimate linking the Euclidean metric on $I$ with the separation time metric on $\Delta$. If $s(x, y)=n$, then $F_{\varepsilon}^{i}\left(\hat{\pi}_{\Delta}(x)\right)$ and $F_{\varepsilon}^{i}\left(\hat{\pi}_{\Delta}(y)\right)$ lie in the same element of $\mathcal{Y}_{\varepsilon}$ for each $i<n$, and $F_{\varepsilon}^{n}\left(\hat{\pi}_{\Delta}(x)\right)$ and $F_{\varepsilon}^{n}\left(\hat{\pi}_{\Delta}(y)\right)$ lie in the same element of $\mathcal{Q}$. Then since $D F_{\varepsilon}^{n} \geqslant C_{e} \sigma^{n}>1$,

$$
\frac{\left|\hat{\pi}_{\Delta}(x)-\hat{\pi}_{\Delta}(y)\right|^{\eta}}{d_{\theta}(x, y)}=\frac{\left|\hat{\pi}_{\Delta}(x)-\hat{\pi}_{\Delta}(y)\right|^{\eta}}{e^{-\theta s(x, y)}} \leqslant \frac{C_{e}^{\eta} \sigma^{-\eta n}}{e^{-\theta n}} .
$$


Choosing $\theta<\eta \log \sigma$ guarantees that a $\eta$-Hölder continuous function on $I$ (and $\hat{I}_{z, \varepsilon_{0}, \varepsilon}$ ) lifts to a Lipschitz function on $\Delta$. Then Lemma 4.6(a) implies the required bounded distortion for $\varphi_{\Delta}$.

(P4) Subexponential Growth of Potential: For each $\delta>0$, there exists $C>0$ such that

$$
\left|S_{R_{\varepsilon}} \varphi_{\Delta}(x)\right| \leqslant C e^{\delta R_{\varepsilon}(x)} \quad \text { for all } x \in \Delta_{0} .
$$

This is immediate for Hölder continuous potentials since $\varphi$ is bounded so

$$
\left|S_{R_{\varepsilon}} \varphi_{\Delta}(x)\right| \leqslant R_{\varepsilon}(x)|\varphi|_{\infty} \quad \text { for all } x \in \Delta_{0}
$$

For geometric potentials, $\varphi=-t \log |D f|-p_{t}$, (P4) is guaranteed by the uniform expansion of $F_{\varepsilon}$ at return times, noting that

$$
S_{R_{\varepsilon}} \varphi_{\Delta}(x)=S_{R_{\varepsilon}} \varphi\left(\hat{\pi}_{\Delta}(x)\right)=-t \log \left|D F_{\varepsilon}\left(\hat{\pi}_{\Delta}(x)\right)\right|-R_{\varepsilon}(x) p_{t} .
$$

By (GM2), $C_{e} \sigma \leqslant\left|D F_{\varepsilon}\right| \leqslant(\sup |D f|)^{R_{\varepsilon}}$, and since $\sup |D f|<\infty$, we have

$$
\left|S_{R_{\varepsilon}} \varphi_{\Delta}(x)\right| \leqslant R_{\varepsilon}(x)\left|p_{t}\right|+t \max \left\{\left|\log \left(C_{e} \sigma\right)\right|, R_{\varepsilon}(x) \log |D f|_{\infty}\right\} \quad \text { for all } x \in \Delta_{0} .
$$

With (P1)-(P4) verified, we are prepared to study the action of $\stackrel{\circ}{\mathcal{L}}_{\Delta}$ on an appropriate function space. Using (P2), choose $\beta$ such that $-\log \bar{\lambda}_{\varepsilon}<\beta<\alpha$. Define a weighted $L^{\infty}$ norm on $\Delta$ by,

$$
\|\psi\|_{\infty}=\sup _{\ell} e^{-\beta \ell} \sup \left\{|\psi(x)|: x \in \Delta_{\ell}\right\},
$$

as well as the weighted Lipschitz norm,

$$
|\psi|_{\text {Lip }}=\sup _{\ell} e^{-\beta \ell} \sup \left\{e^{-\theta s(x, y)}|\psi(x)-\psi(y)|: x, y \in \Delta_{\ell}\right\} .
$$

Then define $\mathcal{B}=\left\{\psi \in L^{1}\left(m_{\Delta}\right):\|\psi\|_{\mathcal{B}}<\infty\right\}$, where $\|\psi\|_{\mathcal{B}}=\|\psi\|_{\infty}+|\psi|_{\text {Lip }}$. We define $\mathcal{B}_{0} \subset \mathcal{B}$ to be the set of bounded functions on $\Delta$ whose Lipschitz constant is also bounded, i.e., $\mathcal{B}_{0}$ uses the same definition as $\mathcal{B}$, but with $\beta=0$. Recall $\varepsilon_{1}^{*}>0$ from Lemma 6.2 and $\varepsilon^{*}>0$ from the verification of (P2).

Theorem 6.3. ([DT2, Theorem 4.12]) Since the open system $\left(f_{\Delta}, \Delta_{;} H_{\Delta}\right)$ is mixing on partition elements and satisfies properties (P1)-(P4), we conclude that $\mathcal{L}_{\Delta}$ has a spectral gap on $\mathcal{B}$ for all $\varepsilon<\min \left\{\varepsilon^{*}, \varepsilon_{1}^{*}\right\}$. Let $\lambda_{\varepsilon}$ denote the largest eigenvalue of $\dot{\mathcal{L}}_{\Delta}$ and let $\stackrel{\circ}{g}_{\Delta}$ denote the corresponding normalised eigenfunction.

(a) The escape rate with respect to $m_{\Delta}$ exists and equals $-\log \lambda_{\varepsilon}$.

(b) $\log \lambda_{\varepsilon}=\sup \left\{h_{\vartheta}\left(f_{\Delta}\right)+\int_{\dot{\Delta}} \varphi_{\Delta}^{H_{\varepsilon}} d \vartheta: \vartheta \in \mathcal{M}_{f_{\Delta}}, \vartheta\left(-\varphi_{\Delta}^{H}\right)<\infty\right\}$, where $\mathcal{M}_{f_{\Delta}}$ is the set of $f_{\Delta^{-}}$ invariant probability measures on $\Delta$.

(c) The following limit defines a probability measure $\nu_{\Delta}$, supported on $\cap_{n=0}^{\infty} \stackrel{\Delta}{ }^{(n)}$,

$$
\nu_{\Delta}(\varphi)=\lim _{n \rightarrow \infty} \lambda_{\varepsilon}^{-n} \int_{\grave{\Delta}^{(n)}} \psi \stackrel{\circ}{g}_{\Delta} d m_{\Delta} \quad \text { for all } \psi \in \mathcal{B}_{0} .
$$

Moreover, the measure $\nu_{\Delta}$ is the unique measure in $\mathcal{M}_{f_{\Delta}}$ that attains the supremum in (b), i.e., it is the unique equilibrium state for $\varphi_{\Delta}^{H_{\varepsilon}}$.

(d) There exist constants $D>0$ and $\sigma_{0}<1$ such that for all $\psi \in \mathcal{B}$,

$$
\left\|\lambda_{\varepsilon}^{-n} \stackrel{\circ}{\mathcal{L}}_{\Delta}^{n} \psi-d(\psi) \stackrel{\circ}{g}_{\Delta}\right\|_{\mathcal{B}} \leq D\|\psi\|_{\mathcal{B}} \sigma_{0}^{n}, \quad \text { where } d(\psi)=\lim _{n \rightarrow \infty} \lambda_{\varepsilon}^{-n} \int_{\dot{\Delta}^{(n)}} \psi d m_{\Delta}<\infty .
$$


Also, for any $\psi \in \mathcal{B}$ with $d(\psi)>0$,

$$
\left|\frac{\stackrel{\circ}{\mathcal{L}}_{\Delta}^{n} \psi}{\left|\dot{\mathcal{L}}_{\Delta}^{n} \psi\right|_{L^{1}\left(m_{\Delta}\right)}}-\stackrel{\circ}{g}_{\Delta}\right|_{L^{1}\left(m_{\Delta}\right)} \leqslant D\|\psi\|_{\mathcal{B} \sigma_{0}^{n}}
$$

6.2. Proof of Theorem 3.1. In this section, we will prove the items of Theorem 3.1 using Theorem 6.3. The following lemma will allow us to lift Hölder continuous functions on $I$ to Lipschitz functions on $\Delta$.

Lemma 6.4. Suppose $\theta / \log \sigma \leqslant \varsigma \leqslant 1$, where $\sigma>1$ is from (GM2). Let $\psi \in C^{\varsigma}(I)$ and define $\tilde{\psi}$ on $\Delta$ by $\tilde{\psi}=\psi \circ \pi_{\Delta}$. Then $|\tilde{\psi}|_{\infty} \leqslant|\psi|_{\infty}$ and Lip $(\tilde{\psi}) \leqslant C|\psi|_{C^{\varsigma}(I)}$ for some constant $C$ depending on the minimum length of elements of $\mathcal{Q}$.

Proof. The bound $|\tilde{\psi}|_{\infty} \leqslant|\psi|_{\infty}$ is immediate. To prove the bound on the Lipschitz constant of $\tilde{\psi}$, suppose $x, y \in \Delta_{\ell, j}$ and estimate,

$$
\frac{|\tilde{\psi}(x)-\tilde{\psi}(y)|}{d_{\theta}(x, y)}=\frac{\left|\psi\left(\pi_{\Delta}(x)\right)-\psi\left(\pi_{\Delta}(y)\right)\right|}{\left|\pi_{\Delta}(x)-\pi_{\Delta}(y)\right|^{\varsigma}} \cdot \frac{\left|\pi_{\Delta}(x)-\pi_{\Delta}(y)\right|^{\varsigma}}{\left|\pi_{\Delta}\left(f_{\Delta}^{R_{\varepsilon}} x\right)-\pi_{\Delta}\left(f_{\Delta}^{R_{\varepsilon}} y\right)\right|^{\varsigma}} \cdot \frac{\left|\pi_{\Delta}\left(f_{\Delta}^{R_{\varepsilon}} x\right)-\pi_{\Delta}\left(f_{\Delta}^{R_{\varepsilon}} y\right)\right|^{\varsigma}}{e^{-\theta s(x, y)}} .
$$

The first ratio above is bounded by $|\psi|_{C^{\varsigma}(I)}$. The second ratio is bounded due to bounded distortion and the backward contraction condition (PolShr $)_{\beta}$ at return times to $Y$. For the third ratio, we use 6.2 , recalling that the separation time only counts returns to $\Delta_{0}$, and that $\theta \leqslant \varsigma \log \sigma$.

In order to project densities from $\Delta$ to $I$, for $\psi \in L^{1}\left(m_{\Delta}\right)$, and $x \in I$, define

$$
\mathcal{P}_{\Delta} \psi(x)=\sum_{y \in \pi_{\Delta}^{-1} x} \frac{\psi(y)}{J \pi_{\Delta}(y)},
$$

where $J \pi_{\Delta}$ is the Jacobian of $\pi_{\Delta}$ with respect to the measures $m_{\varphi}$ and $m_{\Delta}$. Note that for $y \in \Delta_{\ell}$, with $y=f_{\Delta}^{\ell}(z)$ for $z \in \Delta_{0}$, the conformality of $m_{\varphi}$ implies,

$$
\frac{1}{J \pi_{\Delta}(y)}=\frac{d m_{\Delta}(y)}{d m_{\varphi}\left(\pi_{\Delta} y\right)}=\frac{d m_{\Delta}(y)}{d m_{\varphi}\left(\pi_{\Delta}\left(f_{\Delta}^{\ell} z\right)\right)}=\frac{d m_{\varphi}\left(\pi_{\Delta} z\right)}{d m_{\varphi}\left(f^{\ell}\left(\pi_{\Delta} z\right)\right)}=e^{S_{\ell} \varphi\left(\pi_{\Delta} z\right)} .
$$

Then the proof of Lemma 6.4 implies that $1 / J \pi_{\Delta}$ is Lipschitz continuous on each $\Delta_{\ell, j}$ with Lipschitz constant depending only on the level $\ell$.

It follows from the definition of $m_{\Delta}$ that $\mathcal{P}_{\Delta} \psi \in L^{1}\left(m_{\varphi}\right)$, and $\int_{I} \mathcal{P}_{\Delta} \psi d m_{\varphi}=\int_{\Delta} \psi d m_{\Delta}$. Moreover,

$$
\stackrel{\circ}{\mathcal{L}}_{\varphi^{H}{ }_{\varepsilon}}^{n}\left(\mathcal{P}_{\Delta} \psi\right)=\mathcal{P}_{\Delta}\left(\stackrel{\circ}{\mathcal{L}}_{\Delta}^{n} \psi\right) \text {, for each } n \in \mathbb{N} \text {. }
$$

The final step in translating Theorem 6.3 to Theorem 3.1 is the following.

Lemma 6.5. $C^{\varsigma}(\stackrel{\circ}{I}) \subset \mathcal{P}_{\Delta} \mathcal{B}_{0}$ for all $\varsigma \geqslant \theta / \log \sigma$.

Proof. Let $Q \in \mathcal{Q}$ and note that by the leo property there exists $N \in \mathbb{N}$ such that $f^{N}(\pi(Q))=I$. This implies that $\pi\left(\hat{I}_{z, \varepsilon_{0}, \varepsilon}(N)\right)=I$, where $\hat{I}_{z, \varepsilon_{0}, \varepsilon}(N)$ denotes the first $N$ levels of $\hat{I}_{z, \varepsilon_{0}, \varepsilon}$ as in Section 4.4. This in turn implies that $\pi_{\Delta}\left(\cup_{\ell \leqslant N} \Delta_{\ell}\right)=I\left(\bmod 0\right.$ with respect to $\left.m_{\varphi}\right)$.

Next, we select a collection $\mathcal{K}$ of $\Delta_{\ell, i}, \ell \leqslant N$, such that $\pi_{\Delta}\left(\cup_{\Delta_{\ell, i} \in \mathcal{K}} \Delta_{\ell, i}\right)=I$ and $\pi_{\Delta}\left(\Delta_{\ell, i}\right) \cap$ $\pi_{\Delta}\left(\Delta_{\ell^{\prime}, j}\right)=\emptyset$ except for at most finitely many pairs $\Delta_{\ell, i}, \Delta_{\ell^{\prime}, j} \in \mathcal{K}$. Such a collection exists since $f^{N}$ has at most finitely many intervals of monotonicity, so that when the images of two branches overlap, we may eliminate all the $\Delta_{\ell, i}$ in one branch from our set $\mathcal{K}$. The only time when we may be forced to retain two overlapping $\Delta_{\ell, i}$ occurs at the end of one of the branches of monotonicity. 
In this way, we are guaranteed the existence of a set $\mathcal{K}$ with the property that only finitely many elements have projections that overlap.

With the set $\mathcal{K}$ established, the rest of the proof follows along the lines of [BDM, Proposition 4.2]. Essentially, it amounts to inverting the projection operator $\mathcal{P}_{\Delta}$ defined in 6.3 .

Let $\psi \in C^{\varsigma}(I)$ be given. Define $\tilde{\psi} \equiv 0$ on $\Delta \backslash \cup_{\Delta_{\ell, i} \in \mathcal{K}} \Delta_{\ell, i}$. Next, if $\Delta_{\ell, i} \in \mathcal{K}$ and $\pi_{\Delta}\left(\Delta_{\ell, i}\right)$ does not overlap the projection of any other $\Delta_{\ell^{\prime}, j} \in \mathcal{K}$, then for $x \in \Delta_{\ell, i}$, we may define $\tilde{\psi}(x)=$ $\psi\left(\pi_{\Delta} x\right) J \pi_{\Delta}(x)$. It follows that $\mathcal{P}_{\Delta} \tilde{\psi}(x)=\psi(\pi x)$ for $x \in \Delta_{\ell, i}$, and by (6.4) and Lemma 6.4. $\tilde{\psi}$ is Lipschitz with norm depending on the level $\ell$.

Finally, for elements of $\mathcal{K}$ whose projections overlap, we proceed as follows. Suppose $\pi_{\Delta}\left(\Delta_{\ell, i}\right) \cap$ $\pi_{\Delta}\left(\Delta_{\ell^{\prime}, j}\right) \neq \emptyset$. Let $A=\pi_{\Delta}\left(\Delta_{\ell, i}\right) \cup \pi_{\Delta}\left(\Delta_{\ell^{\prime}, j}\right)$ and choose a partition of unity $\left\{\rho_{1}, \rho_{2}\right\}$ for the interval $A$ such that $\rho_{1}, \rho_{2} \in C^{\varsigma}(A)$, and $\rho_{1}=1$ on $\pi_{\Delta}\left(\Delta_{\ell, i}\right) \backslash \pi_{\Delta}\left(\Delta_{\ell^{\prime}, j}\right)$, while $\rho_{2}=1$ on $\pi_{\Delta}\left(\Delta_{\ell^{\prime}, j}\right) \backslash \pi_{\Delta}\left(\Delta_{\ell, i}\right)$.

Define $\tilde{\psi}$ for $x \in \Delta_{\ell, i}$ by

$$
\tilde{\psi}(x)=\psi\left(\pi_{\Delta} x\right) J \pi_{\Delta}(x) \rho_{1}(\pi x),
$$

and similarly define $\tilde{\psi}$ on $\Delta_{\ell^{\prime}, j}$ using $\rho_{2}$. It is clear that $\mathcal{P}_{\Delta} \tilde{\psi}(y)=\psi(y)$ for $y \in A$. This construction using partitions of unity $\rho_{i}$ can be modified to account for finitely may overlaps in $\pi\left(\Delta_{\ell, i}\right), \Delta_{\ell, i} \in \mathcal{K}$, while keeping a uniform bound on the $C^{\varsigma}$-norm of $\rho_{i}$.

In this way, we define $\tilde{\psi}$ on $\Delta_{\ell, i}$ for all $\Delta_{\ell, i} \in \mathcal{K}$. Since $\pi_{\Delta}\left(\cup_{\Delta_{\ell, i} \in \mathcal{K}} \Delta_{\ell, i}\right)=I$, we have $\mathcal{P}_{\Delta} \tilde{\psi}=\psi(\bmod$ $0)$. And since $\mathcal{K}$ contains only elements on level at most $N$, by (6.4) and Lemma 6.4, $\tilde{\psi} \in \mathcal{B}_{0}$.

We proceed to prove the items of Theorem 3.1 .

Recall that $\eta \in(0,1]$ is the relevant Hölder exponent for $\varphi$. For geometric potentials, we take $\eta=1$ due to Lemma 4.6(b). Fix $\varsigma \in(0, \eta]$. Then we may choose $\theta \leqslant \varsigma \log \sigma$, so that Lemma 6.5 holds. Then also $\theta \leqslant \eta \log \sigma$ as required by (P3). Choosing $\beta$ such that $-\log \bar{\lambda}_{\varepsilon}<\beta<\alpha$ then fixes the appropriate Banach space $\mathcal{B}$ for Theorem 6.3. In what follows, we assume $\varepsilon<\min \left\{\varepsilon^{*}, \varepsilon_{1}^{*}\right\}$.

(a) The existence of the escape rate $-\log \lambda_{\varepsilon}$ follows from Theorem 6.3(a) and Lemma 6.1. Define

$$
\stackrel{\circ}{g}_{\varepsilon}=\mathcal{P}_{\Delta} \stackrel{\circ}{g}_{\Delta} .
$$

By (6.5), one has $\stackrel{\circ}{g}_{\varepsilon} \in L^{1}\left(m_{\varphi}\right)$ and for each $n$,

$$
\stackrel{\circ}{\mathcal{L}}_{\varphi}^{n} \stackrel{\circ}{\varepsilon}_{\varepsilon}=\mathcal{P}_{\Delta}\left(\stackrel{\circ}{\mathcal{L}}_{\Delta}^{n} \stackrel{\circ}{g}_{\Delta}\right)=\mathcal{P}_{\Delta}\left(\lambda_{\varepsilon}^{n} \stackrel{\circ}{g}_{\Delta}\right)=\lambda_{\varepsilon}^{n} \stackrel{\circ}{g}_{\varepsilon},
$$

so that $\stackrel{\circ}{g}_{\varepsilon} d m_{\varphi}$ defines a conditionally invariant probability measure on $I$ with eigenvalue $\lambda_{\varepsilon}$.

(b) We define the required conformal measure $m_{H_{\varepsilon}}$, using the by-now standard procedure,

$$
m_{H_{\varepsilon}}(\psi):=\lim _{n \rightarrow \infty} \lambda_{\varepsilon}^{-n} \int_{I^{n}} \psi d m_{\varphi}, \quad \text { for } \psi \in C^{\varsigma}(I) .
$$

Using Lemma 6.5, we find $\tilde{\psi} \in \mathcal{B}_{0}$ such that $\mathcal{P}_{\Delta} \tilde{\psi}=\psi$. Then by (6.5),

$$
\int_{I^{n}} \psi d m_{\varphi}=\int_{I} \dot{\mathcal{L}}_{\varphi^{H}{ }^{n}}^{n} \psi d m_{\varphi}=\int_{\Delta} \stackrel{\mathcal{L}}{\Delta}_{\Delta}^{n} \tilde{\psi} d m_{\Delta}=\int_{\check{\Delta}^{(n)}} \tilde{\psi} d m_{\Delta},
$$

so that the limit in $(6.6)$ exists by Theorem $6.3(\mathrm{~d})$, using the spectral gap enjoyed by $\dot{\mathcal{L}}_{\Delta}$. Indeed, $d(\tilde{\psi})=m_{H_{\varepsilon}}(\psi)$. The fact that $m_{H_{\varepsilon}}$ defined in this way is $\varphi$-conformal follows from the same calculation as in the proof of [DT2, Theorem 1.7]. The fact that $m_{H_{\varepsilon}}$ is supported on $I^{\infty}$ follows from its definition in 6.6 . 
(c) Defining $\nu_{H_{\varepsilon}}:=\stackrel{\circ}{g}_{\varepsilon} m_{H_{\varepsilon}}$, we see that

$$
\nu_{H_{\varepsilon}}(\psi)=\lim _{n \rightarrow \infty} \lambda_{\varepsilon}^{-n} \int_{I^{n}} \stackrel{\circ}{g}_{\varepsilon} \psi d m_{\varphi}, \quad \text { for } \psi \in C^{\varsigma}(I),
$$

since $\mathcal{P}_{\Delta}\left(\stackrel{\circ}{g}_{\Delta} \tilde{\psi}\right)=\stackrel{\circ}{g}_{\varepsilon} \psi$, and $\stackrel{\circ}{g}_{\Delta} \tilde{\psi} \in \mathcal{B}$ by Lemma 6.5 . This extends to $\psi \in C^{0}(I)$ by approximation: for each $\epsilon>0$, we may choose $\psi_{\delta} \in C^{\varsigma}(I)$ such that $\left|\psi-\psi_{\delta}\right|_{C^{0}(I)} \leqslant \epsilon$ and $\left|\psi_{\delta}\right|_{C^{\varsigma}(I)} \leqslant \delta^{-\varsigma}$. (This can be accomplished, for example, through convolution of $\psi$ with a $C^{\infty}$ mollifier.) Then $\nu_{H_{\varepsilon}}\left(\psi_{\delta}-\psi_{\delta^{\prime}}\right) \leqslant 2 \epsilon$ for each $\delta^{\prime}<\delta$, so that $\left(\nu_{H_{\varepsilon}}\left(\psi_{\delta}\right)\right)_{\delta>0}$ forms a Cauchy family as $\delta \rightarrow 0$. Moreover,

$$
\lim _{n \rightarrow \infty} \lambda_{\varepsilon}^{-n} \int_{\check{I}^{n}} \stackrel{\circ}{g}_{\varepsilon} \psi d m_{\varphi}=\lim _{n \rightarrow \infty} \lambda_{\varepsilon}^{-n} \int_{\stackrel{\circ}{I}^{n}} \stackrel{\circ}{g}_{\varepsilon}\left(\psi-\psi_{\delta}\right) d m_{\varphi}+\nu_{H_{\varepsilon}}\left(\psi_{\delta}\right)=\nu_{H_{\varepsilon}}\left(\psi_{\delta}\right)+\mathcal{O}(\epsilon)
$$

since $\lambda_{\varepsilon}^{-n} \int_{I^{n}} \stackrel{\circ}{g}_{\varepsilon} d m_{\varphi}=1$ for each $n \in \mathbb{N}$. Since $\epsilon>0$ was arbitrary, $\nu_{H_{\varepsilon}}(\psi)$ exists and is given by the limit in (6.7).

Next, again using the commutivity given by (6.5), we see that $\nu_{H_{\varepsilon}}=\left(\pi_{\Delta}\right)_{*} \nu_{\Delta}$, where $\nu_{\Delta}$ is from Theorem 6.3(c). It follows that

$$
\log \lambda_{\varepsilon}=h_{\nu_{\Delta}}\left(f_{\Delta}\right)+\int \varphi_{\Delta} d \nu_{\Delta}=h_{\nu_{H_{\varepsilon}}}(f)+\int \varphi d \nu_{H_{\varepsilon}}
$$

since $\pi_{\Delta}: \Delta \rightarrow I$ is at most countable-to-one, so that $\nu_{H_{\varepsilon}}$ achieves the supremum in the variational principle among all invariant probability measures on $i^{\infty}$ that lift to an invariant probability measure on $\Delta$, and $\nu_{H_{\varepsilon}}$ is unique in this class.

In order to conclude that in fact $\nu_{H_{\varepsilon}}$ achieves the supremum over all invariant probability measures $\nu$ with $\nu\left(-\phi^{H_{\varepsilon}}\right)<\infty$, i.e., that are supported on $\stackrel{\circ}{I}^{\infty}$, we note the following inequality, taking our notation from Theorem 4.10 .

$$
P(\phi)-\int \phi d \nu=\int(P(\phi)-\phi) d \nu \geqslant \bar{\alpha}=\alpha+\xi \geqslant \alpha,
$$

for any such measure $\nu$, which follows from the proof of Theorem 4.10 for all classes of our admissible potentials. Note also that $\int \phi^{H_{\varepsilon}} d \nu=\int \phi d \nu$ whenever $\nu\left(-\phi^{H_{\varepsilon}}\right)<\infty$.

By choice of $L$ in Section 4.5, any ergodic invariant measure $\nu$ with entropy $h_{\nu}(f)>\left(\log \lambda_{\varepsilon^{*}}+\alpha\right) / 2$ lifts to our inducing scheme. For an $f$-invariant measure $\nu$ with $\nu\left(-\phi^{H_{\varepsilon}}\right)<\infty$, define the pressure of $\nu$ to be $P_{\nu}\left(\phi^{H_{\varepsilon}}\right)=h_{\nu}(f)+\int \phi d \nu$. Now if $P_{\nu}\left(\phi^{H_{\varepsilon}}\right) \geqslant P_{\nu_{H_{\varepsilon}}}\left(\phi^{H_{\varepsilon}}\right)$, then,

$$
h_{\nu}(f)+\int \phi d \nu-P(\phi) \geqslant h_{\nu_{H_{\varepsilon}}}(f)+\int \phi d \nu_{H_{\varepsilon}}-P(\phi)=\log \lambda_{\varepsilon},
$$

by (6.8) so that $h_{\nu}(f) \geqslant \log \lambda_{\varepsilon}+\alpha \geqslant \log \lambda_{\varepsilon^{*}}+\alpha$, using (6.9), and so $\nu$ lifts to our inducing scheme by our choice of $L$. Thus $P_{\nu}\left(\phi^{H_{\varepsilon}}\right) \leqslant P_{\nu_{H_{\varepsilon}}}\left(\phi^{H_{\varepsilon}}\right)$, and $\nu_{H_{\varepsilon}}$ achieves the supremum among all invariant measures $\nu$ satisfying $\nu\left(-\phi^{H_{\varepsilon}}\right)<\infty$ (so in fact $\nu=\nu_{H_{\varepsilon}}$ ). Thus, $\nu_{H_{\varepsilon}}$ is the unique equilibrium state for $\phi^{H_{\varepsilon}}$, proving item (c) of the theorem.

(d) The characterisation of the limit proving item (d) now follows from Theorem 6.3(d), again using Lemma 6.5 to lift any $\psi \in C^{\varsigma}(\stackrel{\circ}{I})$ to a function $\tilde{\psi} \in B_{0}$, and then evolving that function according to 6.5. The convergence extends to any $\psi \in C^{\varsigma}(I)$ since in one iterate, $\stackrel{\circ}{\mathcal{L}}_{\varphi^{H_{\varepsilon}}} \psi$ is supported on $\stackrel{\circ}{I}$ so the values of $\psi$ on $H_{\varepsilon}=I \backslash \stackrel{\circ}{I}$ are irrelevant to the value of the limit.

To justify Remark 3.2 , note that the convergence in (d) holds for any $\psi \in \mathcal{P}_{\Delta} \mathcal{B}_{0}$ with $\nu_{H_{\varepsilon}}(\psi)>0$ due to 6.5. In particular, since the invariant density $g_{\varphi}=\frac{d \mu_{\varphi}}{d m_{\varphi}}$ satisfies $g_{\varphi}=\mathcal{P}_{\Delta} g_{\Delta}$ for some $g_{\Delta} \in \mathcal{B}_{0}$, for any $\psi \in C^{\varsigma}(I)$, we may define $\tilde{\psi}=\psi \circ \pi_{\Delta}$, and then conclude that $\tilde{\psi} g_{\Delta} \in \mathcal{B}_{0}$ by Lemma 6.4. Thus $\psi g_{\varphi} \in \mathcal{P}_{\Delta} \mathcal{B}_{0}$, and so ${\stackrel{\circ}{\mathcal{L}^{H}{ }^{H}}}^{n}\left(\psi g_{\varphi}\right) /\left|{\stackrel{\mathcal{L}}{\varphi^{H}{ }_{\varepsilon}}}^{n}\left(\psi g_{\varphi}\right)\right|_{L^{1}\left(m_{\varphi}\right)}$ converges to $\stackrel{\circ}{g}_{\varepsilon}$ as $n \rightarrow \infty$. 


\section{ZERO-HOLE LIMIT}

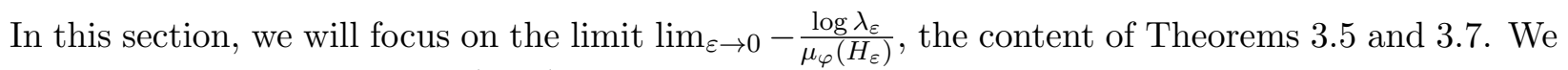
assume throughout that $\varepsilon \in\left(0, \varepsilon_{2}\right)$, so that the conclusions of Corollary 5.5 hold. Indeed, we will use the spectral gap for $\dot{\mathcal{L}}_{\varepsilon}$ to construct a canonical invariant measure $\hat{\nu}_{\varepsilon}$ for $\stackrel{\circ}{F}_{\varepsilon}$, supported on the survivor set, $\stackrel{\circ}{Y}_{\varepsilon}^{\infty}=\cap_{n=0}^{\infty} \stackrel{\circ}{F}_{\varepsilon}^{-n}(Y)$.

For $\psi \in \mathcal{C}^{\eta}(\mathcal{Q})$, define

$$
\hat{\nu}_{\varepsilon}(\psi):=\lim _{n \rightarrow \infty} \Lambda_{\varepsilon}^{-n} \int_{\dot{\Upsilon}_{\varepsilon}^{n-1}} \psi \stackrel{\circ}{G}_{\varepsilon} d \hat{m}
$$

The limit exists since

$$
\Lambda_{\varepsilon}^{-n} \int_{\dot{\check{Y}}_{\varepsilon}^{n-1}} \psi \stackrel{\circ}{G}_{\varepsilon} d \hat{m}=\int_{Y} \Lambda_{\varepsilon}^{-n} \dot{\mathcal{L}}_{\varepsilon}^{n}\left(\psi \stackrel{\circ}{G}_{\varepsilon}\right) d \hat{m} \underset{n \rightarrow \infty}{\longrightarrow} \stackrel{\circ}{\varepsilon}_{\varepsilon}\left(\psi \stackrel{\circ}{G}_{\varepsilon}\right),
$$

where $\stackrel{\circ}{\varepsilon}_{\varepsilon}$ is from Corollary 5.5. Since $\left|\hat{\nu}_{\varepsilon}(\psi)\right| \leqslant \hat{\nu}_{\varepsilon}(1)|\psi|_{\infty}, \hat{\nu}_{\varepsilon}$ extends to a bounded linear functional on $\mathcal{C}^{0}(\mathcal{Q})$, i.e., $\hat{\nu}_{\varepsilon}$ is a Borel measure. Moreover, $\hat{\nu}_{\varepsilon}(1)=1$, so $\nu_{\varepsilon}$ is a probability measure, clearly supported on $\stackrel{\circ}{\varepsilon}_{\varepsilon}^{\infty}$.

Let $\stackrel{\circ}{\Phi}_{\varepsilon}$ denote the punctured version of the induced potential $\Phi_{\varepsilon}$, i.e., $\stackrel{\circ}{\Phi}_{\varepsilon}=\Phi_{\varepsilon}$ on $Y \backslash \hat{H}_{\varepsilon}^{\prime}$, and $\stackrel{\circ}{\Phi}_{\varepsilon}=-\infty$ on $\hat{H}_{\varepsilon}^{\prime}$. Recall $P\left(\Phi_{\varepsilon}\right)=0$ by Remark 4.7. According to [DT2, Section 6.4.1], $\hat{\nu}_{\varepsilon}$ is an equilibrium state for the potential $\stackrel{\circ}{\Phi}_{\varepsilon}-\log \Lambda_{\varepsilon}$; on the other hand, by [BDM, Lemma 5.3], $\hat{\nu}_{\varepsilon}$ is a Gibbs measure for the potential $\stackrel{\circ}{\Phi}_{\varepsilon}-R_{\varepsilon} \log \lambda_{\varepsilon}$, with pressure $P_{\hat{\nu}_{\varepsilon}}\left(\stackrel{\circ}{\Phi}_{\varepsilon}-R_{\varepsilon} \log \Lambda_{\varepsilon}\right)=0$. We conclude,

$$
\log \Lambda_{\varepsilon}=\left(\int R_{\varepsilon} d \hat{\nu}_{\varepsilon}\right) \log \lambda_{\varepsilon}
$$

Recalling $\hat{\mu}_{Y, \varepsilon}=G_{\varepsilon} \hat{m}$ is the invariant probability measure for $F_{z, \varepsilon_{0}, \varepsilon}$, supported on $Y$, Kac's Lemma in 4.4 implies $\hat{\mu}_{\varepsilon}\left(\hat{H}_{\varepsilon}^{\prime}\right)=\frac{\hat{\mu}_{Y, \varepsilon}\left(\hat{H}_{\varepsilon}^{\prime}\right)}{\int R_{\varepsilon} d \hat{\mu}_{Y, \varepsilon}}$. So putting these together yields

$$
\frac{\log \lambda_{\varepsilon}}{\hat{\mu}_{\varepsilon}\left(\hat{H}_{\varepsilon}\right)}=\frac{\log \lambda_{\varepsilon}}{\hat{\mu}_{\varepsilon}\left(\hat{H}_{\varepsilon}^{\prime}\right)} \cdot \frac{\hat{\mu}_{\varepsilon}\left(\hat{H}_{\varepsilon}^{\prime}\right)}{\hat{\mu}_{\varepsilon}\left(\hat{H}_{\varepsilon}\right)}=\frac{\log \Lambda_{\varepsilon}}{\hat{\mu}_{Y, \varepsilon}\left(\hat{H}_{\varepsilon}^{\prime}\right)} \cdot \frac{\int R_{\varepsilon} d \hat{\mu}_{Y, \varepsilon}}{\int R_{\varepsilon} d \hat{\nu}_{\varepsilon}} \cdot \frac{\hat{\mu}_{\varepsilon}\left(\hat{H}_{\varepsilon}^{\prime}\right)}{\hat{\mu}_{\varepsilon}\left(\hat{H}_{\varepsilon}\right)} .
$$

Therefore to prove Theorems 3.5 and 3.7 we must show that as $\varepsilon \rightarrow 0$,

$$
-\frac{\log \Lambda_{\varepsilon}}{\hat{\mu}_{Y, \varepsilon}\left(\hat{H}_{\varepsilon}^{\prime}\right)} \rightarrow 1, \quad \frac{\int R_{\varepsilon} d \hat{\mu}_{Y, \varepsilon}}{\int R_{\varepsilon} d \hat{\nu}_{\varepsilon}} \rightarrow 1, \quad \text { and } \quad \frac{\hat{\mu}_{\varepsilon}\left(\hat{H}_{\varepsilon}^{\prime}\right)}{\hat{\mu}_{\varepsilon}\left(\hat{H}_{\varepsilon}\right)} \rightarrow 1-e^{-S_{p} \varphi(z)}
$$

(we take $e^{-S_{p} \varphi(z)}=0$ when $z$ is aperiodic). These are Theorem 7.2 . Proposition 7.3 and then Lemmas 7.5 and 7.6 in the Hölder case and Lemmas 7.10 and Lemma 7.11 in the geometric case.

7.1. An asymptotic for $\Lambda_{\varepsilon}$. In this subsection, we obtain a precise asymptotic for $\Lambda_{\varepsilon}$ in terms of the quantity $\hat{\mu}_{Y, \varepsilon}\left(\hat{H}_{\varepsilon}^{\prime}\right)$, proving the first limit in $(7.4)$.

We remark that we are not able to apply the results of [KL2] in our setting since it does not fit into the assumptions of that paper. In [KL2], it is assumed that there is a sequence of operators $P_{\varepsilon}$, with a decomposition similar to that given by Corollary 5.5 and having largest eigenvalue $\rho_{\varepsilon}$. These operators approach a fixed operator $P_{0}$ with eigenvalue 1 and the derivative of $\log \rho_{\varepsilon}$ is expressed in terms of the size of the perturbation $P_{0}-P_{\varepsilon}$. 
In our setting, the only candidate for $P_{0}$ is our transfer operator $\mathcal{L}_{0}=\mathcal{L}_{z, \varepsilon_{0}}$, the transfer operator corresponding to $F_{z, \varepsilon_{0}}$, which does not depend on $\varepsilon$. However, the relation between $\delta$ and $\varepsilon$ given by Lemma 5.2 is not explicit, so that a good asymptotic expression for $\Lambda_{\varepsilon}$ is not available starting from $\mathcal{L}_{0}$ (indeed, the relation between $\varepsilon$ and $\delta$ depends in part on the rate of approach of the orbit of $z$ to itself, which is not guaranteed to be proportional to the measure of $\hat{H}_{\varepsilon}^{\prime}$ ). Instead, as suggested by Lemma 5.4 , the difference between $\mathcal{L}_{\varepsilon}$ and $\dot{\mathcal{L}}_{\varepsilon}$ has the correct order for the asymptotic we want. In order to exploit this, we consider then two sequences of operators, $\left(\mathcal{L}_{\varepsilon}\right)_{\varepsilon>0}$ and $\left(\mathcal{L}_{\varepsilon}\right)_{\varepsilon>0}$, and use their uniform spectral properties to prove the required asymptotic for the maximal eigenvalues $\Lambda_{\varepsilon}$ of the latter sequence in terms of the maximal eigenfunctions of the former sequence.

We begin by establishing the following improved regularity for the functions $G_{\varepsilon}$ and $\dot{G}_{\varepsilon}$.

Lemma 7.1. For all $\varepsilon \in\left(0, \varepsilon_{2}\right)$, where $\varepsilon_{2}>0$ is from Corollary 5.5,

$$
H^{\eta}\left(\log G_{\varepsilon}\right) \leqslant C_{d}, \quad \text { and } \quad H^{\eta}\left(\log \stackrel{\circ}{G}_{\varepsilon}\right) \leqslant C_{d} .
$$

As a consequence, there exists $c_{0}>0$ such that for all $\varepsilon \in\left(0, \varepsilon_{2}\right)$,

$$
c_{0} \leqslant \inf _{Y} \dot{\circ}_{\varepsilon} \leqslant\left\|\stackrel{\circ}{G}_{\varepsilon}\right\|_{\mathcal{C}^{\eta}} \leqslant c_{0}^{-1},
$$

and similar bounds hold for $G_{\varepsilon}$.

Proof. Suppose $\psi \in \mathcal{C}^{\eta}$ satisfies $H^{\eta}(\log \psi) \leqslant K$. Then $\psi(x) / \psi(y) \leqslant e^{K d(x, y)^{\eta}}$, for any $x, y$ belonging to the same element of $\mathcal{Q}$.

We follow the notation in the proof of Proposition 5.1. Let $x, y \in Q \in \mathcal{Q}$. For $n \geqslant 0$ and $u \in{\stackrel{\circ}{F_{\varepsilon}}}^{-n}(x)$, let $Y_{i}^{(n)}(u)$ denote the $n$-cylinder containing $u$. For each $u$, there is a unique $v \in \stackrel{\circ}{F}_{\varepsilon}^{-n}(y) \cap Y_{i}^{(n)}(u)$.

Using the log-Hölder regularity of $\psi$ as well as the bounded distortion property (GM3), we estimate,

$$
\begin{aligned}
\stackrel{\circ}{\mathcal{L}}_{\varepsilon}^{n} \psi(x) & =\sum_{u \in \stackrel{\circ}{\varepsilon}_{\varepsilon}^{-n}(x)} \psi(u) e^{S_{n} \Phi_{\varepsilon}(u)} \leqslant \sum_{u \in \stackrel{\circ}{F}_{\varepsilon}^{-n}(x)} \psi(v) e^{K d(u, v)^{\eta}} e^{S_{n} \Phi_{\varepsilon}(v)}\left(1+C_{d} d(x, y)^{\eta}\right) \\
& \leqslant \stackrel{\mathcal{L}}{\varepsilon}_{\varepsilon}^{n} \psi(y) e^{K C_{e}^{-\eta} \sigma^{-n \eta} d(x, y)^{\eta}}\left(1+C_{d} d(x, y)^{\eta}\right),
\end{aligned}
$$

where for the last inequality, we have used property (GM2). Now taking logs, and using the inequality $\log (1+t) \leqslant t$ for all $t \geqslant 0$, we have

$$
H^{\eta}\left(\log \dot{\mathcal{L}}_{\varepsilon}^{n} \psi\right) \leqslant K C_{e}^{-\eta} \sigma^{-n \eta} H^{\eta}(\log \psi)+C_{d}, \quad \text { for all } n \geqslant 1 .
$$

This implies that for $n$ large enough, $\mathcal{L}_{\varepsilon}^{n}$ preserves the set of functions $\left\{\psi \in C^{\eta}(\mathcal{Q}): H^{\eta}(\log \psi) \leqslant\right.$ $\left.1+C_{d}\right\}$. Thus $\stackrel{\circ}{G}_{\varepsilon}$ must belong to this set. Since $\stackrel{\circ}{\mathcal{L}}_{\varepsilon} \stackrel{\circ}{\varepsilon}_{\varepsilon}=\Lambda_{\varepsilon} \stackrel{\circ}{G}_{\varepsilon}$, substituting $\stackrel{\circ}{G}_{\varepsilon}$ into (7.7) and taking $n \rightarrow \infty$ implies that $H^{\eta}\left(\log \stackrel{\circ}{G}_{\varepsilon}\right) \leqslant C_{d}$, proving (7.5).

By a nearly identical argument, (7.7) applies to $\mathcal{L}_{\varepsilon}$ as well, and so its fixed point $G_{\varepsilon}$ satisfies $(7.5)$.

Finally, we show how (7.5) implies (7.6). The uniform upper bounds on $\left|\stackrel{G}{\varepsilon}_{\varepsilon}\right| \mathcal{C}^{\eta}$ and $\left|G_{\varepsilon}\right|_{\mathcal{C}}$ follow immediately from Proposition 5.1. we can set $c_{1}^{-1}=C$ from that proposition, so we focus on the lower bounds.

Since $\int \stackrel{\circ}{G}_{\varepsilon} d \hat{m}=1$, there exists $Q_{0} \in \mathcal{Q}$ such that $\sup _{x \in Q_{0}} \stackrel{\circ}{G}_{\varepsilon}(x) \geqslant 1$. By $(7.5), \inf _{x \in Q_{0}} \stackrel{\circ}{G}_{\varepsilon}(x) \geqslant$ $e^{-C_{d}}$. Now by the mixing property of $F_{\varepsilon}$ together with Lemma 4.12 , there exists $n_{0} \in \mathbb{N}$, independent of $\varepsilon \in\left(0, \varepsilon_{1}\right)$, such that $\hat{f}_{\varepsilon}^{n_{0}}\left(Q_{0}\right) \supset Y$. Thus for any $y \in Y$, there exists $n(y) \leqslant n_{0}$ such that 
$R^{n(y)}(y)=n_{0}$. Then,

$$
\stackrel{\circ}{G}_{\varepsilon}(y)=\Lambda_{\varepsilon}^{-n(y)} \dot{\circ}_{\varepsilon}^{n(y)} \stackrel{\circ}{G}_{\varepsilon}(y) \geqslant \Lambda_{\varepsilon}^{-n(y)} e^{-C_{d}} \inf _{x \in Q_{0} \cap \hat{f}_{\varepsilon}^{-n_{0}}(Y)} e^{S_{n_{0}} \varphi(x)}=: c_{2} .
$$

Let $c_{0}:=\min \left\{c_{1}, c_{2}\right\}$. Note that by our assumptions on $f$, we have $\inf _{x \in Q_{0} \cap \hat{f}_{\varepsilon}^{-n_{0}}(Y)} e^{S_{n_{0}} \varphi(x)}>0$ even when $\varphi$ is of the form $-t \log |D f|-P(-t \log |D f|)$ because the orbit $x, f(x), \ldots, f^{n_{0}-1}(x)$ avoids a neighbourhood of Crit for any $x \in Q_{0} \cap \hat{f}_{\varepsilon}^{-n_{0}}(Y)$ since $n_{0}$ is a return time to $Y$ on this set. Thus $c_{0}$ is strictly positive and is also independent of $\varepsilon$ by Lemma 4.12 . This proves (7.6) for $\stackrel{\circ}{G}_{\varepsilon}$ and an identical argument can be used for $G_{\varepsilon}$.

\section{Theorem 7.2.}

$$
\lim _{\varepsilon \rightarrow 0} \frac{1-\Lambda_{\varepsilon}}{\hat{\mu}_{Y, \varepsilon}\left(\hat{H}_{\varepsilon}^{\prime}\right)}=1
$$

Proof. We assume $\varepsilon \in\left(0, \varepsilon_{2}\right)$ since we are interested in the limit $\varepsilon \rightarrow 0$. Iterating $(5.10)$ for $n \geqslant 1$,

$$
\stackrel{\circ}{\mathcal{L}}_{\varepsilon}^{n} G_{\varepsilon}=\Lambda_{\varepsilon}^{n} \stackrel{\circ}{\varepsilon}_{\varepsilon}\left(G_{\varepsilon}\right) \stackrel{\circ}{G}_{\varepsilon}+\stackrel{\circ}{\mathcal{R}}_{\varepsilon}^{n} G_{\varepsilon} \Longrightarrow \stackrel{\circ}{G}_{\varepsilon}=\frac{1}{\dot{\circ}_{\varepsilon}\left(G_{\varepsilon}\right)}\left(\Lambda_{\varepsilon}^{-n} \stackrel{\circ}{\mathcal{L}}_{\varepsilon}^{n} G_{\varepsilon}-\Lambda_{\varepsilon}^{-n} \dot{\circ}_{\varepsilon}^{n} G_{\varepsilon}\right) .
$$

Using this identity and (5.9), we estimate

$$
\begin{aligned}
1-\Lambda_{\varepsilon} & =\int \stackrel{\circ}{G}_{\varepsilon} d \hat{m}-\int \stackrel{\circ}{\mathcal{L}}_{\varepsilon} \stackrel{\circ}{\varepsilon}_{\varepsilon} d \hat{m}=\int\left(\mathcal{L}_{\varepsilon}-\stackrel{\mathcal{L}}{\varepsilon}_{\varepsilon}\right) \stackrel{G}{G}_{\varepsilon} d \hat{m}=\int_{\hat{H}_{\varepsilon}^{\prime}} \stackrel{\circ}{\varepsilon}_{\varepsilon} d \hat{m} \\
& =\frac{1}{\stackrel{\circ}{e}_{\varepsilon}\left(G_{\varepsilon}\right)} \int_{\hat{H}_{\varepsilon}^{\prime}}\left(\Lambda_{\varepsilon}^{-n} \stackrel{\circ}{\mathcal{L}}_{\varepsilon}^{n} G_{\varepsilon}-\Lambda_{\varepsilon}^{-n} \stackrel{\circ}{\mathcal{R}}_{\varepsilon}^{n} G_{\varepsilon}\right) d \hat{m} \\
& =\frac{1}{\stackrel{\circ}{\varepsilon}_{\varepsilon}\left(G_{\varepsilon}\right)}\left(\int_{\hat{H}_{\varepsilon}^{\prime}} G_{\varepsilon} d \hat{m}-\int_{\hat{H}_{\varepsilon}^{\prime}}\left(1-\Lambda_{\varepsilon}^{-n} \dot{\mathcal{L}}_{\varepsilon}^{n}\right) G_{\varepsilon} d \hat{m}-\int_{\hat{H}_{\varepsilon}^{\prime}} \Lambda_{\varepsilon}^{-n} \stackrel{\mathcal{R}}{\varepsilon}_{\varepsilon}^{n} G_{\varepsilon} d \hat{m}\right) .
\end{aligned}
$$

Using Corollary 5.5, we estimate the third term on the right side of 7.8 by

$$
\left\|\Lambda_{\varepsilon}^{-n} \mathcal{R}_{\varepsilon}^{n} G_{\varepsilon}\right\|_{\mathcal{C}^{\eta}} \leqslant e^{-2 \beta n / 3} \Lambda_{\varepsilon}^{-n}\left\|G_{\varepsilon}\right\|_{\mathcal{C}^{\eta}} \leqslant e^{-\beta n / 3}\left\|G_{\varepsilon}\right\|_{\mathcal{C}^{\eta}} .
$$

Due to (7.6), $\left\|G_{\varepsilon}\right\|_{\mathcal{C}^{\eta}} \leqslant c_{0}^{-1}$ and $G_{\varepsilon} \geqslant c_{0}$ uniformly in $\varepsilon$. Thus

$$
\left|\int_{\hat{H}_{\varepsilon}^{\prime}} \Lambda_{\varepsilon}^{-n} \mathcal{R}_{\varepsilon}^{n} G_{\varepsilon} d \hat{m}\right|=\int_{\hat{H}_{\varepsilon}^{\prime}} G_{\varepsilon} d \hat{m} \cdot \mathcal{O}\left(e^{-\beta n / 3}\right) .
$$

Next, the second term on the right hand side of $(7.8)$ can be rewritten as,

$$
\int_{\hat{H}_{\varepsilon}^{\prime}}\left(1-\Lambda_{\varepsilon}^{-n} \dot{\mathcal{L}}_{\varepsilon}^{n}\right) G_{\varepsilon} d \hat{m}=\left(1-\Lambda_{\varepsilon}^{-n}\right) \int_{\hat{H}_{\varepsilon}^{\prime}} G_{\varepsilon} d \hat{m}+\Lambda_{\varepsilon}^{-n} \int_{\hat{H}_{\varepsilon}^{\prime}}\left(\mathcal{L}_{0}^{n}-\dot{\mathcal{L}}_{\varepsilon}^{n}\right) G_{\varepsilon} d \hat{m}
$$

recalling that $\mathcal{L}_{0}$ is the transfer operator corresponding to $F_{z, \varepsilon_{0}}$ which also has $\hat{m}$ as a conformal measure. Now the maps $F_{z, \varepsilon_{0}}$ and $\stackrel{\circ}{F}_{z, \varepsilon_{0}, \varepsilon}$ differ on the 1 -cylinders contained in $B_{\varepsilon} \cup \hat{H}_{\varepsilon}^{\prime}$, where $B_{\varepsilon}$ is defined in the proof of Lemma 5.2. Thus $F_{z, \varepsilon_{0}}^{n}$ and $\stackrel{\circ}{F}_{z, \varepsilon_{0}, \varepsilon}^{n}$ differ on the $n$-cylinders contained in $B_{\varepsilon, n}^{\prime}:=\left(\cup_{i=0}^{n-1} F_{z, \varepsilon_{0}}^{-i}\left(B_{\varepsilon} \cup \hat{H}_{\varepsilon}^{\prime}\right)\right) \cup\left(\cup_{i=0}^{n-1} F_{z, \varepsilon_{0}, \varepsilon}^{-i}\left(B_{\varepsilon} \cup \hat{H}_{\varepsilon}^{\prime}\right)\right)$. Now following (5.7) and (5.8), we have

$$
\left|\left(\mathcal{L}_{0}^{n}-\dot{\mathcal{L}}_{\varepsilon}^{n}\right) G_{\varepsilon}\right|_{\infty} \leqslant \frac{1+C_{d}}{q} 2 \hat{m}\left(B_{\varepsilon, n}^{\prime}\right)\left|G_{\varepsilon}\right|_{\infty} .
$$


Then the second term on the right side of $(7.8)$ can be bounded by

$$
\left|\int_{\hat{H}_{\varepsilon}^{\prime}}\left(1-\Lambda_{\varepsilon}^{-n} \stackrel{\circ}{\mathcal{L}}_{\varepsilon}^{n}\right) G_{\varepsilon} d \hat{m}\right|=\int_{\hat{H}_{\varepsilon}^{\prime}} G_{\varepsilon} d \hat{m} \cdot \mathcal{O}\left(\left(1-\Lambda_{\varepsilon}^{-n}\right)+\Lambda_{\varepsilon}^{-n} \hat{m}\left(B_{\varepsilon, n}^{\prime}\right)\right),
$$

using (7.6) again to estimate, $\int_{\hat{H}_{\varepsilon}^{\prime}}\left|G_{\varepsilon}\right|_{\infty} d \hat{m} \leqslant c_{0}^{-2} \int_{\hat{H}_{\varepsilon}^{\prime}} G_{\varepsilon} d \hat{m}$.

Putting (7.9) and 7.10 together with (7.8) and dividing through by $\int_{\hat{H}_{\varepsilon}^{\prime}} G_{\varepsilon} d \hat{m}=\hat{\mu}_{Y, \varepsilon}\left(\hat{H}_{\varepsilon}^{\prime}\right)$ yields,

$$
\frac{1-\Lambda_{\varepsilon}}{\hat{\mu}_{Y, \varepsilon}\left(\hat{H}_{\varepsilon}^{\prime}\right)}=\frac{1}{\grave{e}_{\varepsilon}\left(G_{\varepsilon}\right)}\left(1+\mathcal{O}\left(\left(1-\Lambda_{\varepsilon}^{-n}\right)+\Lambda_{\varepsilon}^{-n} \hat{m}\left(B_{\varepsilon, n}^{\prime}\right)+e^{-\beta n / 3}\right)\right) .
$$

The quantity $\stackrel{\circ}{\varepsilon}_{\varepsilon}\left(G_{\varepsilon}\right)$ can be made arbitrarily close to $e_{0}\left(G_{\varepsilon}\right)=1$ by Corollary 5.5 .

Now fix $\delta>0$ and first choose $n$ sufficiently large that $e^{-\beta n / 3}<\delta$. Next choose $\varepsilon$ sufficiently small so that $\left|\stackrel{e}{e}_{\varepsilon}\left(G_{\varepsilon}\right)-1\right|<\delta,\left|1-\Lambda_{\varepsilon}^{-n}\right|<\delta$ and $\Lambda_{\varepsilon}^{-n} \leqslant 2$ by Corollary 5.5, and $\hat{m}\left(B_{n, \varepsilon}^{\prime}\right)<\delta$ by Corollary 4.13. Then the error term is $\mathcal{O}(\delta)$, and since $\delta$ was arbitrary, the theorem follows.

7.2. Convergence of the integral of the return time. In this subsection, we prove the convergence of the second limit in (7.4), regarding the integral of the return time. As before, we assume $\varepsilon \in\left(0, \varepsilon_{2}\right)$, so that the conclusions of Corollary 5.5 hold.

Recall the invariant measure $\hat{\nu}_{\varepsilon}$ from (7.1) supported on $\stackrel{\circ}{Y}^{\infty}$, and that $\hat{\mu}_{Y, \varepsilon}=G_{\varepsilon} \hat{m}$ is the invariant measure for $F_{\varepsilon}$ given by Corollary 5.3 . The main result of this section is the following.

Proposition 7.3. Let $R_{\varepsilon}=R_{z, \varepsilon_{0}, \varepsilon}$. Then,

$$
\lim _{\varepsilon \rightarrow 0} \frac{\int R_{\varepsilon} d \hat{\mu}_{Y, \varepsilon}}{\int R_{\varepsilon} d \hat{\nu}_{\varepsilon}}=1
$$

Proof. First we show that for $\psi \in \mathcal{C}^{\eta}(\mathcal{Q}),\left|\hat{\nu}_{\varepsilon}(\psi)-\hat{\mu}_{Y, 0}(\psi)\right| \rightarrow 0$ as $\varepsilon \rightarrow 0$. Let $\stackrel{\circ}{\varepsilon}_{\varepsilon}$ be the projector defined by $\stackrel{\circ}{\varepsilon}_{\varepsilon} \otimes \stackrel{\circ}{\varepsilon}_{\varepsilon}$, i.e.

$$
\stackrel{\circ}{\Pi}_{\varepsilon}(\psi)=\stackrel{\circ}{e}_{\varepsilon}(\psi) \stackrel{\circ}{G}_{\varepsilon}, \quad \text { for all } \psi \in \mathcal{C}^{\eta}(\mathcal{Q}),
$$

and similarly for $\Pi_{0}$. Recall that we have normalised the eigenvectors so that $\hat{m}\left(\stackrel{\circ}{G}_{\varepsilon}\right)=\hat{m}\left(G_{0}\right)=1$.

Notice that since $\mathcal{L}_{0}^{*} \hat{m}=\hat{m}, e_{0}(\psi)$ is simply $\hat{m}(\psi)$. Thus $\hat{\mu}_{Y, 0}(\psi)=e_{0}\left(\psi G_{0}\right)$. Now,

$$
\begin{aligned}
\left|\hat{\nu}_{\varepsilon}(\psi)-\hat{\mu}_{Y, 0}(\psi)\right| & \leqslant\left|\stackrel{\circ}{\varepsilon}_{\varepsilon}\left(\psi \stackrel{\circ}{G}_{\varepsilon}\right)-e_{0}\left(\psi \dot{\circ}_{\varepsilon}\right)\right|+\left|e_{0}\left(\psi \dot{\circ}_{\varepsilon}\right)-e_{0}\left(\psi g_{0}\right)\right| \\
& \leqslant\left|\int_{Y} \stackrel{\circ}{\Pi}_{\varepsilon}\left(\psi \stackrel{\circ}{G}_{\varepsilon}\right)-\Pi_{0}\left(\psi \dot{\circ}_{\varepsilon}\right) d \hat{m}\right|+\left|\int_{Y} \psi\left(\stackrel{\circ}{G}_{\varepsilon}-G_{0}\right) d \hat{m}\right| \\
& \leqslant\left.||\left|\stackrel{\circ}{\Pi}_{\varepsilon}-\Pi_{0}\right|||\left|\psi \stackrel{G}{G}_{\varepsilon} \|_{\mathcal{C}^{\eta}(\mathcal{Q})}+\right| \psi\right|_{\infty}\left|\dot{G}_{\varepsilon}-G_{0}\right|_{L^{1}(\hat{m})},
\end{aligned}
$$

and both terms go to zero as $\varepsilon \rightarrow 0$ by Corollary 5.5 (which in turn uses [KL1]).

It also follows from Corollary 5.3 , that $\hat{\mu}_{Y, \varepsilon}(\psi) \rightarrow \hat{\mu}_{Y, 0}(\psi)$ as $\varepsilon \rightarrow 0$. Thus by the triangle inequality, $\left|\hat{\nu}_{\varepsilon}(\psi)-\hat{\mu}_{Y, \varepsilon}(\psi)\right| \rightarrow 0$ as $\varepsilon \rightarrow 0$, for all $\psi \in \mathcal{C}^{\eta}(\mathcal{Q})$.

This does not immediately imply the proposition since $R_{\varepsilon} \notin \mathcal{C}^{\eta}(\mathcal{Q})$. However, we claim that $\stackrel{\circ}{\mathcal{L}}_{\varepsilon}\left(R_{\varepsilon}\right) \in \mathcal{C}^{\eta}(\mathcal{Q})$. First, $\dot{\mathcal{L}}_{\varepsilon}\left(R_{\varepsilon}\right)$ is bounded for all $x \in Y$ by

$$
\stackrel{\circ}{\mathcal{L}}_{\varepsilon} R_{\varepsilon}(x)=\sum_{u \in \dot{F}_{\varepsilon}^{-1}(x)} R_{\varepsilon}(u) e^{\Phi_{\varepsilon}(u)} \leqslant \frac{1+C_{d}}{q} \sum_{u \in \dot{F}_{\varepsilon}^{-1}(x)} R_{\varepsilon}(u) \hat{m}\left(Y_{i}(u)\right),
$$


by (5.1), where $Y_{i}(u)$ is the 1-cylinder containing $u$. The last sum is simply bounded by $\hat{m}\left(R_{\varepsilon}\right)=$ $\hat{m}_{\varepsilon}\left(\hat{I}_{\varepsilon}\right)$, since $F_{\varepsilon}$ is a first return map to $Y$ in the Hofbauer extension. This is uniformly bounded in $\varepsilon$ by Theorem 4.10. Next, since $R_{\varepsilon}$ is constant on 1-cylinders, $Y_{i} \in \mathcal{Y}_{\varepsilon}$, using (GM3) the Hölder constant of $\dot{\mathcal{L}}_{\varepsilon}\left(R_{\varepsilon}\right)$ is bounded by,

$$
\stackrel{\circ}{\mathcal{L}}_{\varepsilon} R_{\varepsilon}(x)-\stackrel{\circ}{\mathcal{L}}_{\varepsilon} R_{\varepsilon}(y)=\sum_{u \in \dot{\circ}_{\varepsilon}^{-1}(x)} R_{\varepsilon}(u)\left(e^{\Phi_{\varepsilon}(u)}-e^{\Phi_{\varepsilon}(v)}\right) \leqslant C_{d} d(x, y)^{\eta} \sum_{u \in \dot{F}_{\varepsilon}^{-1}(x)} R_{\varepsilon}(u) e^{\Phi_{\varepsilon}(u)},
$$

for all $x, y \in Q \in \mathcal{Q}$, where each $v \in \stackrel{\circ}{F}_{\varepsilon}^{-1}(y)$ is paired with $u \in \stackrel{\circ}{F}_{\varepsilon}^{-1}(x)$ lying in the same 1-cylinder. The sum is again uniformly bounded in $\varepsilon$ as in (7.11), proving the claim.

It follows that, $\stackrel{\circ}{\mathcal{L}}_{\varepsilon}\left(R_{\varepsilon} \stackrel{\circ}{G}_{\varepsilon}\right) \in \mathcal{C}^{\eta}(\mathcal{Q})$ and by Lemma 7.1, also $\frac{\stackrel{\circ}{\mathcal{L}}_{\varepsilon}\left(R_{\varepsilon} \stackrel{\circ}{G}_{\varepsilon}\right)}{\dot{G}_{\varepsilon}} \in \mathcal{C}^{\eta}(\mathcal{Q})$. Now by 7.1 ,

$$
\lim _{n \rightarrow \infty} \Lambda_{\varepsilon}^{-n} \int_{\dot{Y}_{\varepsilon}^{n}} R_{\varepsilon} \stackrel{\circ}{G}_{\varepsilon} d \hat{m}=\lim _{n \rightarrow \infty} \Lambda_{\varepsilon}^{-n} \int_{\dot{Y}_{\varepsilon}^{n-1}} \frac{\stackrel{\circ}{\mathcal{L}}_{\varepsilon}\left(R_{\varepsilon} \stackrel{\circ}{G}_{\varepsilon}\right)}{\dot{\circ}_{\varepsilon}} \stackrel{\circ}{G}_{\varepsilon} d \hat{m} \underset{n \rightarrow \infty}{\longrightarrow} \hat{\nu}_{\varepsilon}\left(\frac{\stackrel{\circ}{\mathcal{L}}_{\varepsilon}\left(R_{\varepsilon} \stackrel{\circ}{G}_{\varepsilon}\right)}{\Lambda_{\varepsilon} \dot{\circ}_{\varepsilon}}\right) .
$$

Thus $\hat{\nu}_{\varepsilon}\left(R_{\varepsilon}\right)$ exists and is defined by (7.1).

For $N \in \mathbb{N}$, define the truncation $R_{\varepsilon}^{(N)}=\min \left\{R_{\varepsilon}, N\right\}$. For $R_{0}=R_{z, \varepsilon_{0}}$, define $R_{0}^{(N)}$ similarly. By the above arguments, it follows that $\dot{\mathcal{L}}_{\varepsilon}\left(R_{\varepsilon}^{(N)} \stackrel{\circ}{G}_{\varepsilon}\right) \in \mathcal{C}^{\eta}(\mathcal{Q})$ and that $\hat{\nu}_{\varepsilon}\left(R_{\varepsilon}^{(N)}\right)$ exists and is defined by (7.1). Similarly for the complementary function, $\dot{\mathcal{L}}_{\varepsilon}\left(1_{R_{\varepsilon}>N} \cdot R_{\varepsilon}\right) \in \mathcal{C}^{\eta}(\mathcal{Q})$, and $\hat{\nu}_{\varepsilon}\left(1_{R_{\varepsilon}>N} \cdot R_{\varepsilon}\right)$ exists and is defined by (7.1).

Next, we claim that $R_{\varepsilon}$ is uniformly integrable with respect to $\hat{\nu}_{\varepsilon}$; in particular, $\hat{\nu}_{\varepsilon}\left(1_{R_{\varepsilon}>N} \cdot R_{\varepsilon}\right) \rightarrow 0$ as $N \rightarrow \infty$ uniformly in $\varepsilon$. To see this, note that by (7.1),

$$
\begin{aligned}
\hat{\nu}_{\varepsilon}\left(1_{R_{\varepsilon}>N} \cdot R_{\varepsilon}\right) & =\lim _{n \rightarrow \infty} \Lambda_{\varepsilon}^{-n} \int_{Y} \stackrel{\circ}{\mathcal{L}}_{\varepsilon}^{n}\left(1_{R_{\varepsilon}>N} \cdot R_{\varepsilon} \stackrel{\circ}{G}_{\varepsilon}\right) d \hat{m} \leqslant \lim _{n \rightarrow \infty}\left|\Lambda_{\varepsilon}^{-n} \stackrel{\circ}{\mathcal{L}}_{\varepsilon}^{n-1}\left(\dot{\circ}_{\varepsilon}\left(1_{R_{\varepsilon}>N} \cdot R_{\varepsilon} \dot{\circ}_{\varepsilon}\right)\right)\right|_{\infty} \\
& \leqslant C\left|\stackrel{\circ}{\mathcal{L}}_{\varepsilon}\left(1_{R_{\varepsilon}>N} \cdot R_{\varepsilon} \stackrel{\circ}{G}_{\varepsilon}\right)\right|_{\infty},
\end{aligned}
$$

where we have used (5.6) for the last inequality, together with the fact that $\Lambda_{\varepsilon}^{-n} \hat{m}\left(\dot{Y}_{\varepsilon}^{n-1}\right)$ is bounded uniformly in $\varepsilon$ and $n$ by Corollary 5.3 . Then estimating as in (7.11),

$$
\left|\stackrel{\circ}{\mathcal{L}}_{\varepsilon}\left(1_{R_{\varepsilon}>N} \cdot R_{\varepsilon} \stackrel{\circ}{G}_{\varepsilon}\right)(x)\right| \leqslant C \sum_{\substack{u \in \stackrel{\circ}{\varepsilon}^{-1}(x) \\ R_{\varepsilon}(u)>N}} R_{\varepsilon}(u) \hat{m}\left(Y_{i}(u)\right) \leqslant C \sum_{k>N} k \hat{m}\left(R_{\varepsilon}=k\right) \leqslant C^{\prime} e^{-\alpha N}
$$

by Theorem 4.10 , and the claim is proved.

It follows from the proof of Corollary 4.13 , that for each $N>0$, there exists $\varepsilon_{N}>0$ such that for $\varepsilon \leqslant \varepsilon_{N}$, all one cylinders $Y_{i}$ for $F_{\varepsilon}$ with $R_{\varepsilon}\left(Y_{i}\right) \leqslant N$, are also one cylinders for $F_{0}$ with the same return time. This implies that $R_{\varepsilon}^{(N)}=R_{0}^{(N)}$ for $\varepsilon \leqslant \varepsilon_{N}$.

Let $\delta>0$ be arbitrary. Choose $N$ such that $\hat{\nu}_{\varepsilon}\left(R_{\varepsilon}>N\right)<\delta, \hat{\mu}_{Y, \varepsilon}\left(R_{\varepsilon}>N\right)<\delta$, and $\hat{\mu}_{Y, 0}\left(R_{0}>\right.$ $N)<\delta$, for all $\varepsilon<\varepsilon_{1}$, which is possible by the claim and Theorem 4.10 . Then for $\varepsilon \leqslant \varepsilon_{N}$, we have

$$
\hat{\nu}_{\varepsilon}\left(R_{\varepsilon}\right)=\hat{\nu}_{\varepsilon}\left(R_{0}^{(N)}\right)+\mathcal{O}(\delta) \underset{\varepsilon \rightarrow 0}{\longrightarrow} \hat{\mu}_{Y, 0}\left(R_{0}^{(N)}\right)+\mathcal{O}(\delta)=\hat{\mu}_{Y, 0}\left(R_{0}\right)+\mathcal{O}(\delta) .
$$

Similarly,

$$
\hat{\mu}_{Y, \varepsilon}\left(R_{\varepsilon}\right)=\hat{\mu}_{Y, \varepsilon}\left(R_{0}^{(N)}\right)+\mathcal{O}(\delta) \underset{\varepsilon \rightarrow 0}{\longrightarrow} \hat{\mu}_{Y, 0}\left(R_{0}^{(N)}\right)+\mathcal{O}(\delta)=\hat{\mu}_{Y, 0}\left(R_{0}\right)+\mathcal{O}(\delta) .
$$

Since $\delta$ was arbitrary, this proves the proposition. 
7.3. Final step of the proof of Theorem 3.5; the Hölder continuous case. In the next two sections, we prove the third limit in (7.4) in both the periodic and nonperiodic cases. In the present section we address the case when $\varphi$ is Hölder continuous, and in Section 7.4 we will address the case when $\varphi$ is a geometric potential. As a prelimary result, we prove the following lemma.

Lemma 7.4. For $f \in \mathcal{F}$ and a Hölder potential $\varphi$, we have $\inf _{x \in I} \frac{d \mu_{\varphi}}{d m_{\varphi}}(x)>0$, where $\mu_{\varphi}$ and $m_{\varphi}$ are the relevant invariant and conformal measures.

Proof. For simplicity we write $g(x)=\frac{d \mu_{\varphi}}{d m_{\varphi}}(x)$ and note that $m_{\varphi}$ is a $\varphi$-conformal measure so $\mathcal{L}_{\varphi} g=g$, where $\mathcal{L}_{\varphi}$ is the transfer operator associated to $\varphi$ and $f$ (not the induced dynamics), defined in Section 2.3. Since $\pi_{*} \hat{\mu}_{\varepsilon}=\mu_{\varphi}$ (we take any $\varepsilon \in\left(0, \varepsilon_{2}\right)$ ), Lemma 7.1 implies that there is an open set $U$ such that $\inf _{x \in U} g(x)>0$. By leo, there is some $n \in \mathbb{N}$ such that $f^{n}(U)=I$. Hence for any $x \in I$ we can estimate

$$
g(x)=\mathcal{L}_{\varphi}^{n} g(x)=\sum_{y \in f^{-n}(x)} g(y) e^{S_{n} \varphi(y)} \geqslant \sum_{y \in\left\{f^{-n}(x)\right\} \cap U} g(y) e^{S_{n} \varphi(y)},
$$

So we conclude by noting that $\inf S_{n} \varphi>-\infty$.

We first address the case in which $z$ is aperiodic.

Lemma 7.5. Let $z$ be an aperiodic point for $f$ and suppose $\varphi$ is Hölder continuous. Then,

$$
\lim _{\varepsilon \rightarrow 0} \frac{\hat{\mu}_{\varepsilon}\left(\hat{H}_{\varepsilon}^{\prime}\right)}{\hat{\mu}_{\varepsilon}\left(\hat{H}_{\varepsilon}\right)}=1
$$

Proof. Recall from (4.4) and 4.5) that $\hat{\mu}_{Y, \varepsilon}$ and $\hat{\mu}_{\varepsilon}$ are related by the following: $\hat{\mu}_{Y, \varepsilon}=\frac{\left.\hat{\mu}_{\varepsilon}\right|_{Y}}{\hat{\mu}_{\varepsilon}(Y)}$, and

$$
\hat{\mu}_{\varepsilon}(A)=\sum_{i} \sum_{j=0}^{R_{i}-1} \hat{\mu}_{\varepsilon}\left(Y_{i} \cap \hat{f}^{-j} A\right), \text { for any Borel } A \subset \hat{I} .
$$

We will apply the above expression to $A=\hat{H}_{\varepsilon}$. Note that due to our construction of $\hat{I}_{z, \varepsilon_{0}, \varepsilon}$, for each $j$ if $\hat{f}^{j}\left(Y_{i}\right) \cap \hat{H}_{\varepsilon} \neq \emptyset$, then $\hat{f}^{j}\left(Y_{i}\right) \subset \hat{H}_{\varepsilon}$. Thus each term in the above sum is either 0 or $\hat{\mu}_{\varepsilon}\left(Y_{i}\right)$. Define for $k \geqslant 1$,

$$
\hat{H}_{\varepsilon}^{\prime}(k)=\left\{Y_{i} \subset \hat{H}_{\varepsilon}^{\prime}: Y_{i} \text { enters } \hat{H}_{\varepsilon} \text { exactly } k \text { times before time } R_{i}\right\} .
$$

Now using (7.13) and our observation about $Y_{i}$,

$$
\hat{\mu}_{\varepsilon}\left(\hat{H}_{\varepsilon}\right)=\sum_{k \geqslant 1} \sum_{Y_{i} \in \hat{H}_{\varepsilon}^{\prime}(k)} k \hat{\mu}_{\varepsilon}\left(Y_{i}\right)=\hat{\mu}_{\varepsilon}\left(\hat{H}_{\varepsilon}^{\prime}\right)+\sum_{k \geqslant 2} \sum_{Y_{i} \in \hat{H}_{\varepsilon}^{\prime}(k)}(k-1) \hat{\mu}_{\varepsilon}\left(Y_{i}\right) .
$$

We proceed to estimate the double sum over $k$ and $Y_{i}$.

By 7.13 , since $F_{\varepsilon}$ is the first return map to $Y$ in $\hat{I}$, the invariant density $G_{\varepsilon}$ from Corollary 5.3 is also the density for $\hat{\mu}_{\varepsilon}$ on $Y$, up to a normalising constant. Applying the uniform bounds on $G_{\varepsilon}$ from Lemma 7.1, we replace $\hat{\mu}_{\varepsilon}\left(Y_{i}\right)$ with $\hat{m}\left(Y_{i}\right)$ in $(7.15)$, up to a uniform constant. For $Y_{i} \subset \hat{H}_{\varepsilon}^{\prime}(k)$, let $T_{i}$ denote the time of the $k$ th entry of $Y_{i}$ to $\hat{H}_{\varepsilon}$ under iteration of $\hat{f}$. By the conformality of $\hat{m}=\hat{m}_{\hat{\varphi}}$,

$$
\hat{m}_{\varepsilon}\left(Y_{i}\right) \leqslant C e^{S_{T_{i}} \hat{\varphi}\left(y_{i}\right)} \hat{m}_{\varepsilon}\left(\hat{f}^{T^{i}} Y_{i}\right) \leqslant C e^{-\bar{\alpha} T_{i}} \hat{m}_{\varepsilon}\left(\hat{f}^{T_{i}} Y_{i}\right),
$$

for any $y_{i} \in Y_{i}$, where $\bar{\alpha}>0$ is from the proof of Theorem 4.10 . 
Fixing $Y_{i} \in \hat{H}_{\varepsilon}^{\prime}(k)$, we wish to estimate $\#\left\{Y_{j} \in \hat{H}_{\varepsilon}^{\prime}(k): T_{j}=T_{i}\right.$ and $\left.\hat{f}^{T_{i}} Y_{i} \cap \hat{f}^{T_{j}} Y_{j} \neq \emptyset\right\}$. Due to our construction of the Hofbauer extension, such a $Y_{j}$ is contained in a set $Z_{j} \in \hat{\mathcal{P}}_{T_{j}+L}$, such that $f^{T_{j}}$ maps $Z_{j}$ injectively into a connected component of $\hat{H}_{\varepsilon} . Z_{j}$ can be associated with a word of length $T_{j}+L$, the first symbol of which lies in $Y$, while the remaining lie in $\hat{I}_{z, \varepsilon_{0}, \varepsilon} \backslash Y$. We divide this word into blocks of length $L$, and note there are $\left\lfloor\frac{T_{j}}{L}\right\rfloor$ of them. They are all external blocks according to the terminology of [DoT]. According to [DoT, Lemma 4.6], there are at most $16 \mathfrak{d}^{2} L^{3}$ external blocks of length $L$. In addition, since $f^{T_{j}} Z_{j} \subset \hat{H}_{\varepsilon}$, we may choose $\varepsilon$ sufficiently small that any remaining symbols between $\left\lfloor\frac{T_{j}}{L}\right\rfloor L$ and $T_{j}$ also belong to an external block of length $L$. Finally, there are at most $(2 \mathfrak{d} L)^{2}$ choices for the first symbol of $Z_{j}$ since this is an upper bound on the number of elements in $\hat{I}_{z, \varepsilon_{0}, \varepsilon}^{\prime}(L)$. Putting these estimates together, we conclude that

$$
\#\left\{Y_{j} \in \hat{H}_{\varepsilon}^{\prime}(k): T_{j}=T_{i} \text { and } \hat{f}^{T_{i}} Y_{i} \cap \hat{f}^{T_{j}} Y_{j} \neq \emptyset\right\} \leqslant(2 \mathfrak{d} L)^{2}\left(16 \mathfrak{d}^{2} L^{3}\right)^{\frac{T_{j}}{L}+1} \leqslant C e^{\xi T_{j}},
$$

where $\xi<\bar{\alpha}$ (by choice of $L$ ) is the same as in the proof of Theorem 4.10 .

Next, due to the aperiodicity of $z$ and the continuity of $f$, for each $\varepsilon>0$ there exists $N=N(\varepsilon) \in \mathbb{N}$, such that $\hat{f}^{j} \hat{H}_{\varepsilon} \cap \hat{H}_{\varepsilon}=\emptyset$ for all $j<N$, and $N(\varepsilon) \rightarrow \infty$ as $\varepsilon \rightarrow 0$. This implies in particular that if $Y_{i} \in \hat{H}_{\varepsilon}^{\prime}(k)$, then $T_{i} \geqslant(k-1) N$.

We organise our estimate for $Y_{i} \subset \hat{H}_{\varepsilon}^{\prime}(k)$ by considering $\hat{H}_{\varepsilon}^{\prime}(k)=\cup_{t \geqslant(k-1) N}\left\{Y_{i} \in \hat{H}_{\varepsilon}^{\prime}(k): T_{i}=t\right\}$. Then using (7.16) and (7.17),

$$
\sum_{\substack{Y_{i} \in \hat{H}_{\varepsilon}^{\prime}(k) \\ T_{i}=t}}(k-1) \hat{\mu}_{\varepsilon}\left(Y_{i}\right) \leq \sum_{\substack{Y_{i} \in \hat{H}_{\varepsilon}^{\prime}(k) \\ T_{i}=t}} C(k-1) e^{-\bar{\alpha} t} \hat{m}_{\varepsilon}\left(\hat{f}^{t}\left(Y_{i}\right)\right) \leq C(k-1) e^{-(\bar{\alpha}-\xi) t}(t+L)^{3} m_{\varphi}\left(H_{\varepsilon}\right),
$$

where for the last inequality, we have used the fact that $\hat{f}^{t}\left(Y_{i}\right)$ lies in a component of $\hat{H}_{\varepsilon}$ in level at most $t+L$ in the Hofbauer extension. Since there are at most $\mathfrak{d} \ell^{2}$ connected components on level $\ell$ according to the proof of [DoT, Lemma 4.6], we obtain that projecting $\left.\hat{H}_{\varepsilon}\right|_{\text {level } \ell}$ down to $H_{\varepsilon}$, we have $\hat{m}_{\varepsilon}\left(\left.\hat{H}_{\varepsilon}\right|_{\text {level } \ell}\right) \leqslant \mathfrak{d} \ell^{2} m_{\varphi}\left(H_{\varepsilon}\right)$ and summing over $\ell \leqslant(t+L)$ yields the required bound.

Using this estimate in the double sum in (7.15), we obtain,

$$
\begin{aligned}
\sum_{k \geqslant 2} \sum_{Y_{i} \subset \hat{H}_{\varepsilon}^{\prime}(k)}(k-1) \hat{\mu}_{\varepsilon}\left(Y_{i}\right) & \leqslant \sum_{k \geqslant 2} \sum_{t \geqslant N(k-1)} \sum_{\substack{Y_{i} \in \hat{H}_{\varepsilon}^{\prime}(k) \\
T_{i}=t}} C e^{-\bar{\alpha} t}(k-1) \hat{m}_{\varepsilon}\left(\hat{f}^{t} Y_{i}\right) \\
& \leqslant \sum_{k \geqslant 2} \sum_{t \geqslant N(k-1)} C e^{-(\bar{\alpha}-\xi) t}(k-1)(t+L)^{3} m_{\varphi}\left(H_{\varepsilon}\right) \\
& \leqslant C^{\prime} m_{\varphi}\left(H_{\varepsilon}\right) \sum_{k \geqslant 2} e^{-\alpha N(k-1)}(k-1) \leqslant C^{\prime \prime} \mu_{\varphi}\left(H_{\varepsilon}\right) e^{-\alpha N}
\end{aligned}
$$

where in the last step we have used Lemma 7.4. Combining this estimate with 7.15 and dividing through by $\hat{\mu}_{\varepsilon}\left(\hat{H}_{\varepsilon}\right)$ (using that $\hat{\mu}_{\varepsilon}\left(\hat{H}_{\varepsilon}\right)=\mu_{\varphi}\left(H_{\varepsilon}\right)$ ) yields,

$$
\frac{\hat{\mu}_{\varepsilon}\left(\hat{H}_{\varepsilon}^{\prime}\right)}{\hat{\mu}_{\varepsilon}\left(\hat{H}_{\varepsilon}\right)}=1-\mathcal{O}\left(e^{-\alpha N}\right) \text {. }
$$

Since $N(\varepsilon) \rightarrow \infty$ as $\varepsilon \rightarrow 0$, this completes the proof of the lemma.

Our next lemma addresses the case in which $z$ is periodic with prime period $p$.

Lemma 7.6. Suppose $z$ is a periodic point for $f$ of prime period $p$, and that $\varphi$ is Hölder continuous. 
a) If $\left\{f^{n}(c): c \in\right.$ Crit, $\left.n \geqslant 1\right\} \cap\{z\}=\emptyset$, then $\lim _{\varepsilon \rightarrow 0} \frac{-\log \lambda_{\varepsilon}}{\mu_{\varphi}\left(H_{\varepsilon}\right)}=1-e^{S_{p} \varphi(z)}$.

b) Suppose $\left\{f^{n}(c): c \in\right.$ Crit, $\left.n \geqslant 1\right\} \cap\{z\} \neq \emptyset$. If in addition, either $f^{p}$ is orientation preserving in a neighbourhood of $z$, or $\lim _{\varepsilon \rightarrow 0} \frac{m_{\varphi}(z+\varepsilon, z)}{m_{\varphi}(z, z-\varepsilon)}=1$, then $\lim _{\varepsilon \rightarrow 0} \frac{-\log \lambda_{\varepsilon}}{\mu_{\varphi}\left(H_{\varepsilon}\right)}=1-e^{S_{p} \varphi(z)}$.

Proof. Fix $N_{0}$ arbirarily large. Due to (4.7), we may choose $\varepsilon^{\prime}>0$ sufficiently small so that for all $\varepsilon<\varepsilon^{\prime}$, the following properties hold.

(i) If $y \in H_{\varepsilon}(z)$, then for all $j=1, \ldots, p N_{0}, f^{j}(y) \in H_{\varepsilon}(z)$ only if $j=k p$ for some $k=1, \ldots N_{0}$.

(ii) If $y \in H_{\varepsilon}(z)$ and there exists $k_{1} \leqslant N_{0}$ such that $f^{k_{1} p}(y) \in H_{\varepsilon}$, then $f^{k p}(y) \in H_{\varepsilon}(z)$ for all $k=1, \ldots, k_{1}$.

(iii) Each 1-cylinder $Y_{i} \subset \hat{H}_{\varepsilon}^{\prime}$ whose first entry time $\ell$ to $\hat{H}_{\varepsilon}$ is less than $N_{0}$ is contained in an interval $Z_{j} \subset Q \in \mathcal{Q}$ such that $\hat{f}^{\ell}\left(Z_{j}\right)$ maps injectively onto a connected component of $\hat{H}_{\varepsilon}$, which we will denote by $\hat{H}_{\varepsilon}\left(Z_{j}\right)$.

(iv) $\hat{f}^{p N_{0}}$ is injective and continuous on each connected component of $\hat{H}_{\varepsilon} \cap \hat{f}^{-p N_{0}}\left(\hat{H}_{\varepsilon}\right)$ that occurs below level $N_{0}$ in $\hat{I}_{z, \varepsilon_{0}, \varepsilon}$.

Properties (i) and (ii) follow from the periodicity of $z$ and the uniform continuity of $f^{n}$ for each orbit segment of length $n \leqslant p N_{0}$. To deduce Property (iii), since $f^{k}\left(z \pm \varepsilon_{0}\right)=z$ is not allowed by choice of $\varepsilon_{0}$ in (4.7), it suffices to choose

$$
\varepsilon^{\prime} \leqslant \frac{1}{2} \min \left\{d\left(z, f^{k}(w)\right): w \in \operatorname{Crit}_{z, \varepsilon_{0}}, f^{k}(w) \neq z, k \leqslant N_{0}\right\} .
$$

With this choice of $\varepsilon^{\prime}$, no boundary points of $\hat{I}_{z, \varepsilon_{0}, \varepsilon}$ for $\varepsilon<\varepsilon^{\prime}$ may fall in the interior of a connected component of $\hat{H}_{\varepsilon}$ with a first entry time less than $N_{0}$. Finally, Property (iv) holds since the orbit of $z$ must be disjoint from Crit; otherwise $f$ would have an attracting periodic orbit, which is forbidden in our class of maps $\mathcal{F}$. Thus we may choose

$$
\varepsilon^{\prime} \leqslant\left|D f^{p N_{0}}\right|_{\infty}^{-1} \min \left\{d\left(f^{k}(z), \text { Crit }\right): k=0, \ldots, p-1\right\}
$$

in order to guarantee (iv).

Starting from (7.13), we group the 1-cylinders $Y_{i} \subset \hat{H}_{\varepsilon}^{\prime}$ as follows. Let $\ell_{i} \in \mathbb{N}$ denote the greatest $\ell \leqslant N_{0}$ such that $f^{\ell p}\left(Y_{i}\right) \subset \hat{H}_{\varepsilon}$. By (i) and (ii) above, if $j \leqslant p N_{0}$, then $\hat{f}^{j}\left(Y_{i}\right) \subset \hat{H}_{\varepsilon}$ if and only if $j=\ell p$ for some $\ell \leqslant \ell_{i}$. Recalling (7.14), we let $\hat{H}_{\varepsilon}^{\prime}\left(k, N_{0}\right)$ denote the set of $Y_{i} \subset \hat{H}_{\varepsilon}^{\prime}(k)$ such that the first entry of $Y_{i}$ to $\hat{H}_{\varepsilon}$ occurs before time $N_{0}$, while $\hat{H}_{\varepsilon}^{\prime}(k, \sim)=\hat{H}_{\varepsilon}^{\prime}(k) \backslash \hat{H}_{\varepsilon}^{\prime}\left(k, N_{0}\right)$. Moreover, $\hat{H}_{\varepsilon}^{\prime}\left(*, N_{0}\right):=\cup_{k \geqslant 1} \hat{H}_{\varepsilon}^{\prime}\left(k, N_{0}\right)$. Then,

$$
\hat{\mu}_{\varepsilon}\left(\hat{H}_{\varepsilon}\right)=\sum_{\ell=1}^{N_{0}} \sum_{\substack{Y_{i} \subset \hat{H}_{\varepsilon}^{\prime}\left(*, N_{0}\right) \\ \ell_{i}=\ell}} \ell \hat{\mu}_{\varepsilon}\left(Y_{i}\right)+\sum_{k>N_{0}} \sum_{Y_{i} \in \hat{H}_{\varepsilon}^{\prime}\left(k, N_{0}\right)}\left(k-\ell_{i}\right) \hat{\mu}_{\varepsilon}\left(Y_{i}\right)+\sum_{k \geqslant 1} \sum_{Y_{i} \in \hat{H}_{\varepsilon}^{\prime}(k, \sim)} k \hat{\mu}_{\varepsilon}\left(Y_{i}\right) .
$$

Since the entry times to $\hat{H}_{\varepsilon}$ are greater than $N_{0}$ for each of the sets counted in the second and third sums above, we may use (7.16) and (7.18) to estimate that these two sums are of order $\mathcal{O}\left(e^{-\alpha N_{0}} \hat{\mu}_{\varepsilon}\left(\hat{H}_{\varepsilon}\right)\right)$. It remains to estimate the first sum above. We rewrite 7.19$)$ as,

$$
\hat{\mu}_{\varepsilon}\left(\hat{H}_{\varepsilon}\right)=\sum_{\ell=1}^{N_{0}} \sum_{\substack{Y_{i} \subset \hat{H}_{\varepsilon}^{\prime}\left(*, N_{0}\right) \\ \ell_{i} \geqslant \ell}} \hat{\mu}_{\varepsilon}\left(Y_{i}\right)+\mathcal{O}\left(e^{-\alpha N_{0}} \hat{\mu}_{\varepsilon}\left(\hat{H}_{\varepsilon}\right)\right) .
$$


For $\ell=1$, we have simply,

$$
\sum_{\substack{Y_{i} \subset \hat{H}_{\varepsilon}^{\prime}\left(*, N_{0}\right) \\ \ell_{i} \geqslant 1}} \hat{\mu}_{\varepsilon}\left(Y_{i}\right)=\hat{\mu}_{\varepsilon}\left(\hat{H}_{\varepsilon}^{\prime}\right)+\mathcal{O}\left(e^{-\alpha N_{0}} \hat{\mu}_{\varepsilon}\left(\hat{H}_{\varepsilon}\right)\right),
$$

since any $Y_{i} \subset \hat{H}_{\varepsilon}^{\prime}$ not counted in the sum for $\ell=1$ has first entry time to $\hat{H}_{\varepsilon}$ greater than $N_{0}$.

To estimate the contribution for the terms corresponding to $\ell=2$, we use property (iii) above. If $Y_{i} \subset \hat{H}_{\varepsilon}\left(*, N_{0}\right)$ with $\ell_{i} \geqslant 2$, then $Y_{i}$ is contained in an interval $Z_{j}$ such that $\hat{f}^{k}\left(Z_{j}\right)$ maps injectively onto a connected component of $\hat{H}_{\varepsilon}$ (for the first time) at some time $k=k\left(Z_{j}\right) \leqslant N_{0}$. Let us denote this component of $\hat{H}_{\varepsilon}$ by $\hat{H}_{\varepsilon}\left(Z_{j}\right)$. Let $A_{j}$ denote those indices $i$ for which $Y_{i} \subset Z_{j}$. Then,

$$
\sum_{\substack{Y_{i} \subset \hat{H}_{\varepsilon}^{\prime}\left(*, N_{0}\right) \\ \ell_{i} \geqslant 2}} \hat{\mu}_{\varepsilon}\left(Y_{i}\right)=\sum_{Z_{j}} \hat{\mu}_{\varepsilon}\left(Z_{j}\right) \sum_{i \in A_{j}} \frac{\hat{\mu}_{\varepsilon}\left(Y_{i}\right)}{\hat{\mu}_{\varepsilon}\left(Z_{j}\right)} .
$$

Notice that since $\ell=2$, for each $i \in A_{j}, \hat{f}^{k\left(Z_{j}\right)}\left(Y_{i}\right) \subset \hat{H}_{\varepsilon}\left(Z_{j}\right) \cap \hat{f}^{-p}\left(\hat{H}_{\varepsilon}\right)$. Recalling Lemma 7.1 and the conformality of $\hat{m}_{\varepsilon}$ (recall that $\hat{m}=\hat{m}_{\varepsilon}$ depends on $\varepsilon$ on $\hat{I}_{z, \varepsilon_{0}, \varepsilon} \backslash Y$ ), we estimate ${ }^{5}$

$$
\begin{aligned}
\sum_{i \in A_{j}} \frac{\hat{\mu}_{\varepsilon}\left(Y_{i}\right)}{\hat{\mu}_{\varepsilon}\left(Z_{j}\right)} & =\sum_{i \in A_{j}} e^{ \pm 2 C_{d} \operatorname{diam}\left(Z_{j}\right)^{\eta}} \frac{\int_{\hat{f}^{k}\left(Y_{i}\right)} e^{S_{k} \hat{\varphi} \circ \hat{f}_{j}^{-k}} d \hat{m}_{\varepsilon}}{\int_{\hat{f}^{k}\left(Z_{j}\right)} e^{S_{k} \hat{\varphi} \circ \hat{f}_{j}^{-k}} d \hat{m}_{\varepsilon}} \\
& =e^{ \pm 2 C_{d} \operatorname{diam}\left(Z_{j}\right)^{\eta}} P_{\varepsilon}\left(S_{k} \hat{\varphi}\left(Z_{j}\right)\right)^{ \pm 2} \frac{\hat{m}_{\varepsilon}\left(\hat{H}_{\varepsilon}\left(Z_{j}\right) \cap \hat{f}^{-p}\left(\hat{H}_{\varepsilon}\right)\right)}{\hat{m}_{\varepsilon}\left(\hat{H}_{\varepsilon}\left(Z_{j}\right)\right)}
\end{aligned}
$$

where $\hat{f}_{j}^{-k}$ is the inverse branch of $\left.\hat{f}^{k}\right|_{Z_{j}}$ and

$$
P_{\varepsilon}\left(S_{k} \hat{\varphi}\left(Z_{j}\right)\right)=\sup _{x, y \in Z_{j}} e^{S_{k} \hat{\varphi}(x)-S_{k} \hat{\varphi}(y)} .
$$

Recall that since we cut at $f^{-1}(z)$ and $f^{-1}(z \pm \varepsilon)$, during our construction of $\hat{I}_{z, \varepsilon_{0}, \varepsilon}, \hat{H}_{\varepsilon}\left(Z_{j}\right)$ must satisfy either $\pi\left(\hat{H}_{\varepsilon}\left(Z_{j}\right)\right)=(z, z+\varepsilon)$ or $\pi\left(\hat{H}_{\varepsilon}\left(Z_{j}\right)\right)=(z-\varepsilon, z)$. Let us denote these intervals above half the hole by $\hat{H}_{\varepsilon}\left(Z_{j}\right)^{+}$or $\hat{H}_{\varepsilon}\left(Z_{j}\right)^{-}$, accordingly. Since $\hat{f}^{p}$ is continuous and injective on $\hat{H}_{\varepsilon}\left(Z_{j}\right)$ by (iv), $\hat{f}^{p}\left(\hat{H}_{\varepsilon}\left(Z_{j}\right) \cap \hat{f}^{-p}\left(\hat{H}_{\varepsilon}\right)\right)$ contains a full interval in the fibre above half the hole (possibly different from $\hat{H}_{\varepsilon}\left(Z_{j}\right)$ ), which we can also denote by + or - as appropriate. Note that the conformal measure of all the lifts of the right half hole $(z, z+\varepsilon)$ have the same measure, and so do all the lifts of the left half hole.

We proceed to prove item (b) of the lemma first. If $f^{p}$ is orientation preserving at $z$, then using conformality and bounded distortion, we have on either half of the hole,

$$
\hat{m}_{\varepsilon}\left(\hat{H}_{\varepsilon}\left(Z_{j}\right)^{ \pm} \cap \hat{f}^{-p}\left(\hat{H}_{\varepsilon}\right)\right)=P_{\varepsilon}\left(S_{p} \varphi\left(H_{\varepsilon}(z)\right)\right)^{ \pm 1} e^{S_{p} \varphi(z)} \hat{m}_{\varepsilon}\left(\hat{H}_{\varepsilon}\left(Z_{j}\right)^{ \pm}\right),
$$

where we have used the fact that $S_{p} \hat{\varphi}(z)=S_{p} \varphi(z)$. On the other hand, if $f^{p}$ is orientation reversing at $z$, then we are left with, for example, the right half hole mapping onto the left half,

$$
\hat{m}_{\varepsilon}\left(\hat{H}_{\varepsilon}\left(Z_{j}\right)^{+} \cap \hat{f}^{-p}\left(\hat{H}_{\varepsilon}\right)\right)=P_{\varepsilon}\left(S_{p} \varphi\left(H_{\varepsilon}(z)\right)\right)^{ \pm 1} e^{S_{p} \varphi(z)} \hat{m}_{\varepsilon}\left(\hat{H}_{\varepsilon}\left(Z_{j}\right)^{-}\right),
$$

and so to conclude the desired cancellation in 7.21 , we use the assumption $\lim _{\varepsilon \rightarrow 0} \frac{m_{\varphi}(z, z+\varepsilon)}{m_{\varphi}(z-\varepsilon, z)}=1$.

\footnotetext{
${ }^{5}$ We use the notation $a=C^{ \pm 1} b$ to mean $C^{-1} b \leqslant a \leqslant C b$ for some constant $C \geqslant 1$.
} 
Thus under either alternative in item (b), we combine the estimates in (7.21) to write,

$$
\begin{aligned}
& \sum_{\substack{Y_{i} \subset \hat{H}_{\varepsilon}^{\prime}\left(*, N_{0}\right) \\
\ell_{i} \geqslant 2}} \hat{\mu}_{\varepsilon}\left(Y_{i}\right)=\sum_{Z_{j}} \hat{\mu}_{\varepsilon}\left(Z_{j}\right) e^{ \pm 2 C_{d} \operatorname{diam}\left(Z_{j}\right)^{\eta}} P_{\varepsilon}\left(S_{k} \hat{\varphi}\left(Z_{j}\right)\right)^{ \pm 2} P_{\varepsilon}\left(S_{p} \varphi\left(H_{\varepsilon}(z)\right)\right)^{ \pm 1} e^{S_{p} \varphi(z)} \\
& =e^{ \pm 2 C_{d} \max _{j} \operatorname{diam}\left(Z_{j}\right)^{\eta}} P_{\varepsilon}\left(S_{k} \hat{\varphi}\left(Z_{j}\right)\right)^{ \pm 2} P_{\varepsilon}\left(S_{p} \varphi\left(H_{\varepsilon}(z)\right)\right)^{ \pm 1} e^{S_{p} \varphi(z)} \hat{\mu}_{\varepsilon}\left(H_{\varepsilon}^{\prime}\right)+\mathcal{O}\left(e^{-\alpha N_{0}} \hat{\mu}_{\varepsilon}\left(\hat{H}_{\varepsilon}\right)\right) .
\end{aligned}
$$

Analogous estimates follow for each $\ell \geqslant 3$. Then using that $e^{S_{\ell p} \varphi(z)}=e^{\ell S_{p}(z)}$, we estimate 7.20 .

$$
\begin{aligned}
& \hat{\mu}_{\varepsilon}\left(\hat{H}_{\varepsilon}\right)=\sum_{\ell=1}^{N_{0}} e^{ \pm 2 C_{d} \max _{j} \operatorname{diam}\left(Z_{j}\right)^{\eta}} P_{\varepsilon}\left(S_{k} \hat{\varphi}\left(Z_{j}\right)\right)^{ \pm 2} P_{\varepsilon}\left(S_{\ell p} \varphi\left(H_{\varepsilon}(z)\right)\right)^{ \pm 1} e^{\ell S_{p} \varphi(z)} \hat{\mu}_{\varepsilon}\left(H_{\varepsilon}^{\prime}\right) \\
& +\mathcal{O}\left(N_{0} e^{-\alpha N_{0}} \hat{\mu}_{\varepsilon}\left(\hat{H}_{\varepsilon}\right)\right) .
\end{aligned}
$$

Since $N_{0}$ is fixed, $Z_{j} \subset Y$ and the first entry of $Z_{j}$ to $\hat{H}_{\varepsilon}$ occurs before time $N_{0}$, we have $\max _{j} \operatorname{diam}\left(Z_{j}\right) \rightarrow 0$ as $\varepsilon \rightarrow 0$. In addition, both $P_{\varepsilon}\left(S_{k} \hat{\varphi}\left(Z_{j}\right)\right)$ and $P_{\varepsilon}\left(S_{\ell p} \varphi\left(H_{\varepsilon}(z)\right)\right)$ approach 1 as $\varepsilon \rightarrow 0$ since the lengths of the orbit segments are uniformly bounded by $p N_{0}$ and $\hat{\varphi}$ is continuous along each orbit segment. Dividing by $\hat{\mu}_{\varepsilon}\left(H_{\varepsilon}\right)$ and taking the limit $\varepsilon \rightarrow 0$ yields for each $N_{0}>0$,

$$
1=\sum_{\ell=1}^{N_{0}} e^{\ell S_{p} \varphi(z)} \lim _{\varepsilon \rightarrow 0} \frac{\hat{\mu}_{\varepsilon}\left(\hat{H}_{\varepsilon}^{\prime}\right)}{\hat{\mu}_{\varepsilon}\left(\hat{H}_{\varepsilon}\right)}+\mathcal{O}\left(N_{0} e^{-\alpha N_{0}}\right) .
$$

Finally, taking $N_{0} \rightarrow \infty$ proves item (b) of the lemma.

The proof of item (a) proceeds similarly starting from (7.21). Now, however, since $z$ is disjoint from the post-critical orbit, we may choose $\varepsilon>0$ sufficiently small that $f^{n}(c) \notin H_{\varepsilon}$ for all $n \leqslant p N_{0}$ and $c \in$ Crit. Then the interval $Z_{j}$ from (iii) can be chosen so that $\pi\left(\hat{f}^{k\left(Z_{j}\right)}\left(Z_{j}\right)\right)=(z-\varepsilon, z+\varepsilon)$, i.e. $\hat{f}^{k\left(Z_{j}\right)}\left(Z_{j}\right)$ covers a level of the fibre above the full hole. Thus we may combine the left and right halves of the hole to obtain the analogue of $(7.22)$ in this case,

$$
\hat{m}_{\varepsilon}\left(\hat{H}_{\varepsilon}\left(Z_{j}\right) \cap \hat{f}^{-p}\left(\hat{H}_{\varepsilon}\right)\right)=P_{\varepsilon}\left(S_{p} \varphi\left(H_{\varepsilon}(z)\right)\right)^{ \pm 1} e^{S_{p} \varphi(z)} \hat{m}_{\varepsilon}\left(\hat{H}_{\varepsilon}\left(Z_{j}\right)\right),
$$

and the orientation preseving character of $f^{p}$ at $z$ is irrelevant. The proof of item (a) of the lemma is then complete, following 7.23 and 7.24 precisely as written.

Now Lemmas 7.5 and 7.6, together with Theorem 7.2 and Proposition 7.3 , complete the proof of Theorem 3.5, via 7.3$)$.

7.4. Final step of the proof of Theorem 3.7 the geometric case. In this section, we prove the third limit in (7.4) in the case when $\varphi=-t \log |D f|-p_{t}, t \in\left(t^{-}, t_{1}\right)$, where $t_{1}$ is defined by (7.28). We assume the slow approach condition (3.3) as well as the polynomial growth condition on the derivative along the post-critical orbit $(3.2)$, formulated in Section 3.2 .2

We first prove an analogue of Lemma 7.4 in this case.

Lemma 7.7. If $f \in \mathcal{F}, \varphi=-t \log |D f|-p_{t}$ and $z$ satisfies (3.3) with $t \in\left(t^{-}, t_{1}\right)$, then there exists $\zeta>0$ such that for all $\varepsilon>0$ sufficiently small, $\inf _{x \in H_{\varepsilon}(z)} \frac{d \mu_{\varphi}}{d m_{\varphi}}(x) \geqslant \zeta$, where $\mu_{\varphi}$ and $m_{\varphi}$ are the relevant invariant and conformal measures.

Proof. The proof is nearly identical to that of Lemma 7.4: While for the geometric potential with $t<0$ it may be that $\inf _{x \in I} S_{n} \varphi(x)=-\infty$, the slow approach condition (3.3) ensures that for $x \in H_{\varepsilon}$, there is a finite lower bound on $S_{n} \varphi(x)$ that is uniform in $\varepsilon$, since $n$ is fixed and independent of $\varepsilon$ in 7.12. 
In order to prove the required convergence for geometric potentials, we will use the setup and notation of [BLS]. It follows from (3.2) and our choice of $\gamma_{n}$, that

$$
\sum_{n} \gamma_{n}<\infty \quad \text { and } \quad \sum_{n \geqslant 1}\left(\gamma_{n}^{d-1} D_{n}(c)\right)^{-1 / d}<\infty \quad \text { for all } c \in \text { Crit } .
$$

This is precisely the condition ${ }^{6}$ required of $f$ in $[\mathrm{BLS}]$.

We will not need the full strength of the results from [BLS]; rather, we will use the estimates on the recovery times for expansion for orbits that pass close to the set Crit. To this end, for a value of $\delta>0$ to be specified later, we define $B_{\delta}$ (Crit) $=\cup_{c \in \text { Crit }}(c-\delta, c+\delta)$, for $\delta>0$. A key estimate of [BLS] is the following.

Lemma 7.8. [BLS, Lemma 2.4] For $\delta>0$ sufficiently small, there exist constants $C_{\delta}, \beta_{\delta}>0$ such that every orbit segment $\left\{f^{i}(x)\right\}_{i=0}^{k-1}$ such that $\left\{f^{i}(x)\right\}_{i=0}^{k-1} \cap B_{\delta}$ (Crit) $=\emptyset$, we have

$$
\left|D f^{k}(x)\right| \geqslant C_{\delta} e^{\beta_{\delta} k} \text {. }
$$

If, in addtion, $f^{k}(x) \in B_{\delta}$ (Crit), then there exists $\kappa>0$ independent of $\delta$ such that,

$$
\left|D f^{k}(x)\right| \geqslant \max \left\{\kappa, C_{\delta} e^{\beta_{\delta} k}\right\} .
$$

Next, we define the notion of binding period recalling the sequence $\left(\gamma_{n}\right)_{n \in \mathbb{N}}$ from (3.2). If $x \in$ $B_{\delta}($ Crit), then

$$
b(x)=\max \left\{b \in \mathbb{N}:\left|f^{k}(x)-f^{k}(c)\right| \leqslant \gamma_{k} \mid f^{k}(c)-\text { Crit } \mid \quad \forall k \leqslant b-1\right\},
$$

and $b(x)=0$ if $x \notin B_{\delta}$ (Crit). Let $I_{b}=\{x \in I: b(x)=b\}$ denote the level sets of $b$. The binding period will be useful in estimating the following important quantity, defined for each $c \in$ Crit,

$$
D f_{\min }^{b}(c):=\min \left\{\left|D f^{b}(x)\right|: x \in I_{b} \cap B_{\delta}(c)\right\},
$$

which governs the minimum rate of growth in expansion along orbit segments.

Note that $b_{\delta}=\min \left\{b(x): x \in B_{\delta}(\right.$ Crit $\left.)\right\}$ tends to $\infty$ as $\delta \rightarrow 0$. This fact is used in [BLS] to make $D f_{\min }^{b_{\delta}}(c)$ arbitrarily large by choosing $\delta>0$ sufficiently small.

Each orbit of length $n$ is assigned an itinerary $\left(\nu_{1}, b_{1}\right),\left(\nu_{2}, b_{2}\right), \ldots,\left(\nu_{k}, b_{k}\right)$, where each $\nu_{i}=\nu_{i}(x)$ represents the first time larger than $\nu_{i-1}+b_{i-1}$ such that the orbit of $x \in I$ makes a return to $B_{\delta}$ (Crit). Each return $i$ is called a deep return and placed in a set $S_{d}=S_{d}(x)$ if the orbit enters $B_{\delta}($ Crit $)$ at time $\nu_{i}$; it is called a shallow return and placed in a set $S_{s}(x)$ if the orbit does not enter $B_{\delta}$ (Crit) at time $\nu_{i}$, but is part of a dynamically defined interval that intersects $B_{\delta}($ Crit).

The key estimates from [BLS] using the information from binding periods are as follows.

Lemma 7.9. BLS, Lemmas 2.5 and 3.2]

a) There exists $C_{0}>0$ independent of $\delta>0$ such that for all $c \in$ Crit and $b \geqslant b_{\delta}$ with $I_{b} \neq \emptyset$,

$$
D f_{\min }^{b}(c) \geqslant C_{0}\left(\gamma_{b}^{d-1} D_{b}(c)\right)^{1 / d}
$$

b) There exist $K_{0}>0$ and $\rho \in(0,1)$, independent of $\delta$, such that for an orbit $\left\{f^{i}(x)\right\}_{i=0}^{n-1}$ with a given sequence $\left(\nu_{1}, b_{1}\right), \ldots,\left(\nu_{k}, b_{k}\right)$ at time $n \geqslant \nu_{k}+b_{k}$, we have

$$
\left|D f^{n}(x)\right| \geqslant \max \left\{C_{\delta}^{\# S_{d}} e^{\beta_{\delta}\left(n-\sum_{i=0}^{k} b_{i}\right)},\left(\frac{\kappa}{K_{0}}\right)^{\# S_{d}} \rho^{-\# S_{s}}\right\} \prod_{i \in S_{d}} D f_{\min }^{b_{i}}\left(c_{i}\right),
$$

\footnotetext{
${ }^{6}$ Indeed, this condition is equivalent to the simpler condition, $\sum_{n \geqslant 1} D_{n}(c)^{-1 /(2 d-1)}<\infty$ BLS, Lemma 2.1], but we use the formulation above in order to directly apply the results of [BLS]. Our condition $[3.2$ is slightly stronger and generalises the exponent to values of $s_{t}<1$.
} 
where $c_{i}$ is the critical point associated to the return at time $\nu_{i}$.

With these key estimates recalled, we are ready to begin our proofs of the relevant limits. As in Section 7.3 , we begin with the aperiodic case.

Lemma 7.10. Suppose $f \in \mathcal{F}_{d}$ and $\phi=-t \log |D f|$ for $t \in\left(t^{-}, t_{1}\right)$ satisfies $(3.2)$. Let $z$ be an aperiodic point for $f$ satisfying (3.3). Then

$$
\lim _{\varepsilon \rightarrow 0} \frac{\hat{\mu}_{\varepsilon}\left(\hat{H}_{\varepsilon}^{\prime}\right)}{\hat{\mu}_{\varepsilon}\left(\hat{H}_{\varepsilon}\right)}=1 .
$$

Proof. We will follow the strategy of the proof of Lemma 7.5, using the same notation defined there. Following (7.15), we must show as before that

$$
\sum_{k \geqslant 2} \sum_{Y_{i} \subset \hat{H}_{\varepsilon}^{\prime}(k)}(k-1) \hat{\mu}_{\varepsilon}\left(Y_{i}\right)=o\left(\hat{\mu}_{\varepsilon}\left(\hat{H}_{\varepsilon}\right)\right) .
$$

However, the estimate in this case is not so simple since the analogous expression to 7.16 does not enjoy uniform exponential contraction in $T_{i}$. Rather, we split $Y_{i} \subset \hat{H}_{\varepsilon}^{\prime}(k)$ into those cylinders which are 'bound' (i.e., in the midst of a binding period) at the time $T_{i}$ of their $k^{\text {th }}$ entry to $\hat{H}_{\varepsilon}$, and those cylinders which are not bound, which we call 'free.'

As before, we fix $N \in \mathbb{N}$ and choose $\varepsilon$ sufficiently small that $\hat{f}^{j}\left(\hat{H}_{\varepsilon}\right) \cap \hat{H}_{\varepsilon}=\emptyset$, for all $j<N$.

Estimate on free pieces. To estimate the contribution to 7.26 from cylinders that are free at time $T_{i}$, we begin as in 7.16,

$$
\hat{\mu}_{\varepsilon}\left(Y_{i}\right)=C^{ \pm 1} \hat{m}_{\varepsilon}\left(Y_{i}\right)=C^{ \pm 1} e^{S_{T_{i}} \hat{\varphi}\left(y_{i}\right)} \hat{m}_{\varepsilon}\left(\hat{f}^{T_{i}} Y_{i}\right)=C^{ \pm 1}\left|D f^{T_{i}}\left(y_{i}\right)\right|^{-t} e^{-p_{t} T_{i}} \hat{m}_{\varepsilon}\left(\hat{f}^{T_{i}} Y_{i}\right) .
$$

We estimate the above expression differently depending whether $t<1$ or $t \geqslant 1$. In all cases, we fix $\delta>0$ sufficiently small that $D f_{\min }^{b_{\delta}}\left(c_{i}\right) \geqslant 2 K_{0} / \kappa$.

If $t<1$, then we consider the following two cases, depending on the itinerary $\left(\nu_{1}, b_{1}\right), \ldots,\left(\nu_{k_{i}}, b_{k_{i}}\right)$ associated to $y_{i}$ from time 0 until time $T_{i}$. Since $\hat{f}^{T_{i}}\left(Y_{i}\right)$ is free, we have $T_{i} \geqslant \nu_{k_{i}}+b_{k_{i}}$ so we may apply Lemma 7.9. Choose $\epsilon>0$ sufficiently small that $\epsilon \leqslant \min \left\{\frac{1}{4}, \frac{-\beta_{\delta} b_{\delta}}{4 \log C_{\delta}}\right\}$.

Case 1. $\sum_{j=0}^{k_{i}} b_{j}>\epsilon T_{i}$. Using the second estimate in Lemma 7.9 (b), we have by choice of $\delta$,

$$
\left|D \hat{f}^{T_{i}}\left(y_{i}\right)\right| \geqslant 2^{\# S_{d}}=2^{k_{i}} .
$$

Case 2. $\sum_{j=0}^{k_{i}} b_{j}<\epsilon T_{i}$. It follows that $\# S_{d}\left(y_{i}\right)=k_{i} \leqslant \epsilon T_{i} / b_{\delta}$. Thus using the first estimate in Lemma 7.9(b), we have by our choice of $\epsilon$,

$$
\left|D \hat{f}^{T_{i}}\left(y_{i}\right)\right| \geqslant C_{\delta}^{\epsilon T_{i} / b_{\delta}} e^{\beta_{\delta} T_{i}(1-\epsilon)} \geqslant e^{\beta_{\delta} T_{i} / 2} .
$$

In either case, our estimate in 6.6 for $t<1$ becomes,

$$
\hat{m}\left(Y_{i}\right) \leqslant C e^{-b_{t} T_{i}} \hat{m}\left(\hat{f}^{T_{i}} Y_{i}\right) .
$$

In the statement of Theorem 3.7 we only consider the case $t \geqslant 1$ under a $(\mathrm{CE})$ condition along the critical orbits:

$$
\exists C, \gamma>0 \text { s.t. } D_{n}(c) \geqslant C e^{\gamma n} \text {. }
$$


In this case, it suffices that $\gamma_{n} \geqslant e^{-\gamma n /(2(d-1))}$, so that $D f_{\min }^{b}(c) \geqslant C_{0} e^{\gamma b /(2 d)}$ by Lemma 7.9 (a). For this range of $t \geqslant 1$, we consider two slightly different cases. Using the same choice of $\delta$ as above, we choose $\epsilon=-\bar{\beta} / \log \left(C_{\delta} C_{0}\right)$, where $\bar{\beta}=\frac{1}{2} \min \left\{\beta_{\delta}, \gamma /(2 d)\right\}$.

Case 1. $k_{i}=\# S_{d}\left(y_{i}\right) \geqslant \epsilon T_{i}$. Using the second estimate in Lemma 7.9 (b) and our choice of $\delta$,

$$
\left|D \hat{f}^{T_{i}}\left(y_{i}\right)\right| \geqslant\left(\frac{\kappa}{K_{0}}\right)^{k_{i}} \prod_{j \in S_{d}\left(y_{i}\right)} D f_{\min }^{b_{j}}\left(c_{j}\right) \geqslant 2^{\epsilon T_{i}}
$$

Case 2. $k_{i} \leqslant \epsilon T_{i}$. Using the first estimate in Lemma 7.9 (b), we have using our choice of $\epsilon$,

$$
\left|D \hat{f}^{T_{i}}\left(y_{i}\right)\right| \geqslant C_{\delta}^{\epsilon T_{i}} e^{\beta_{\delta}\left(T_{i}-\sum_{j=0}^{k_{i}} b_{j}\right)} C_{0}^{\epsilon T_{i}} e^{\gamma \sum_{j=0}^{k_{i}} b_{j} /(2 d)} \geqslant e^{\bar{\beta} T_{i}}
$$

In either case, our estimate in 6.6) for $t \geqslant 1$ becomes,

$$
\hat{m}\left(Y_{i}\right) \leqslant C e^{-\left(t \beta_{1}+p_{t}\right) T_{i}} \hat{m}\left(\hat{f}^{T_{i}} Y_{i}\right)
$$

where $e^{\beta_{1}}=\min \left\{e^{\bar{\beta}}, 2^{\epsilon}\right\}$.

To unify notation, set $\hat{\alpha}=p_{t}$ when $t<1$ in all cases, and $\hat{\alpha}=t \beta_{1}+p_{t}$ in the (CE) case when $t \geqslant 1$. Recall that we defined $t_{1}=1$ in the non- $(\mathrm{CE})$ case; in the $(\mathrm{CE})$ case set

$$
t_{1}:=\sup \left\{t \in\left(1, t^{+}\right): t \beta_{1}+p_{t}>0\right\},
$$

noting that since $p_{1}=0$, such a $t_{1}>1$ exists by continuity of $p_{t}$.

Now the above estimates in conjunction with the complexity estimate 7.17 yield by 7.18 ,

$$
\sum_{\substack{k \geqslant 2 \\ Y_{i} \subset \hat{H}_{\varepsilon}^{\prime}(k) \\ Y_{i} \text { free }}}(k-1) \hat{\mu}_{\varepsilon}\left(Y_{i}\right) \leqslant \sum_{k \geqslant 2} \sum_{j \geqslant N(k-1)} C e^{-(\hat{\alpha}-\xi) j}(k-1)(j+L)^{3} m_{\varphi}\left(H_{\varepsilon}\right) \leqslant C^{\prime} \hat{\mu}_{\varepsilon}\left(\hat{H}_{\varepsilon}\right) e^{-(\hat{\alpha}-\xi) N},
$$

where we may choose $L$ sufficiently large that $\xi<\hat{\alpha}$, and in the second inequality we have used Lemma 7.7 and the fact that $\hat{\mu}_{\varepsilon}\left(\hat{H}_{\varepsilon}\right)=\mu_{\varphi}\left(H_{\varepsilon}\right)$.

Estimate on bound pieces. Next we estimate the contribution to 7.26 from cylinders $Y_{i}$ which are undergoing a bound period at time $T_{i}$. Let $\nu_{i}$ denote the time that $Y_{i}$ enters this bound period. By assumption, $\nu_{i} \leqslant T_{i}<\nu_{i}+b_{i}$. Let $x \in \hat{f}^{\nu_{i}}\left(Y_{i}\right) \subset B_{\delta}(c)$. Then using the slow approach condition (3.3) and the definition of $b$,

$$
\delta_{z} \gamma_{T_{i}-\nu_{i}}^{1-\theta} \leqslant\left|\hat{f}^{T_{i}-\nu_{i}}(c)-z\right| \leqslant\left|\hat{f}^{T_{i}-\nu_{i}}(c)-\hat{f}^{T_{i}-\nu_{i}}(x)\right|+\left|\hat{f}^{T_{i}-\nu_{i}}(x)-z\right| \leqslant \gamma_{T_{i}-\nu_{i}}+\frac{1}{2}\left|H_{\varepsilon}(z)\right| .
$$

This implies that

$$
\delta_{z} \leqslant 2 \max \left\{\gamma_{T_{i}-\nu_{i}}^{\theta}, \frac{1}{2}\left|H_{\varepsilon}(z)\right| \gamma_{T_{i}-\nu_{i}}^{\theta-1}\right\}
$$

We consider the ways in which this can be satisfied. First,

$$
\delta_{z} \leqslant 2 \gamma_{T_{i}-\nu_{i}}^{\theta} \Longrightarrow \gamma_{T_{i}-\nu_{i}} \geqslant\left(\delta_{z} / 2\right)^{1 / \theta} .
$$

Since $\gamma_{n}$ is summable, this condition can be satisfied by only finitely many values of $T_{i}-\nu_{i}$, that depend only on $\gamma_{n}, \delta_{z}$ and $\theta$. Indeed, we can render this set empty since (3.3) implies $\left\{f^{n}(c)\right\}_{n \geqslant 0} \cap\{z\}=\emptyset$. So by choosing $\varepsilon, \delta>0$ sufficiently small, we can make $f^{k}\left(B_{\delta}(\right.$ Crit $\left.)\right)$ disjoint from $H_{\varepsilon}$ for these finitely many iterates.

Next, the second possibility is that

$$
\delta_{z} \leqslant\left|H_{\varepsilon}(z)\right| \gamma_{T_{i}-\nu_{i}}^{\theta-1} \Longrightarrow \gamma_{T_{i}-\nu_{i}} \leqslant\left(\frac{\left|H_{\varepsilon}(z)\right|}{\delta_{z}}\right)^{1 /(1-\theta)} .
$$


Recall from Section 3.2 .2 that we defined $\gamma_{n}=n^{-r}$ for some $r>\frac{1}{s_{t}(1-\theta)}$. Then 7.30 implies

$$
T_{i}-\nu_{i} \geqslant\left(\frac{\delta_{z}}{\left|H_{\varepsilon}(z)\right|}\right)^{1 / r(1-\theta)} .
$$

This implies that the return time to $Y$ for $Y_{i}$ satisfies

$$
R_{i} \geqslant \max \left\{(k-1) N, T_{i}\right\} \geqslant \frac{1}{2}\left((k-1) N+\left(\frac{\delta_{z}}{\left|H_{\varepsilon}(z)\right|}\right)^{1 / r(1-\theta)}\right)=: \tau_{\varepsilon},
$$

where the first condition comes from the fact that $Y_{i} \subset \hat{H}_{\varepsilon}^{\prime}(k)$ and $N$ comes from the aperiodicity condition on $z$. Thus using Theorem 4.10 ,

$$
\begin{aligned}
& \sum_{\substack { k \geqslant 2 \\
\begin{subarray}{c}{Y_{i} \subset \hat{H}_{\varepsilon}^{\prime}(k) \\
Y_{i} \text { bound }{ k \geqslant 2 \\
\begin{subarray} { c } { Y _ { i } \subset \hat { H } _ { \varepsilon } ^ { \prime } ( k ) \\
Y _ { i } \text { bound } } }\end{subarray}}(k-1) \hat{\mu}_{\varepsilon}\left(Y_{i}\right) \leqslant \sum_{k \geqslant 2} \sum_{j \geqslant \tau_{\varepsilon}} \sum_{R_{i}=j}(k-1) \hat{\mu}_{\varepsilon}\left(Y_{i}\right) \leqslant \sum_{k \geqslant 2} \sum_{j \geqslant \tau_{\varepsilon}} C(k-1) e^{-\alpha j} \\
& \leqslant \sum_{k \geqslant 2}(k-1) C^{\prime} e^{-\alpha(k-1) N / 2} e^{-\frac{\alpha}{2}\left(\frac{\delta_{z}}{H_{\varepsilon}(z) \mid}\right)^{1 / r(1-\theta)}}=o\left(e^{-\alpha N / 2}\left|H_{\varepsilon}(z)\right|^{s}\right)=o\left(\hat{\mu}_{\varepsilon}\left(\hat{H}_{\varepsilon}\right)\right),
\end{aligned}
$$

where $s>0$ represents any positive power, and the switch to $\hat{\mu}_{\varepsilon}\left(H_{\varepsilon}\right)$ is possible due to the scaling exponent $s_{t}$ for the conformal measure $m_{\varphi}$ as well as Lemma 7.7.

Combining (7.29) and (7.31) proves (7.26), which by (7.15) completes the proof of the lemma.

Next, we address the case when $z$ is periodic with prime period $p$. We continue to assume the slow approach condition (3.3).

Lemma 7.11. Suppose $f \in \mathcal{F}_{d}$ and $\phi=-t \log |D f|$ for $t \in\left(t^{-}, t_{1}\right)$ satisfies (3.2). Let $z$ be $a$ periodic point for $f$ of prime period $p$ satisfying $(3.3)$. Then

$$
\lim _{\varepsilon \rightarrow 0} \frac{\hat{\mu}_{\varepsilon}\left(\hat{H}_{\varepsilon}^{\prime}\right)}{\hat{\mu}_{\varepsilon}\left(\hat{H}_{\varepsilon}\right)}=1-e^{S_{p} \varphi(z)} .
$$

Proof. We follow the proof of Lemma 7.6, which needs few modifications now that we have recorded the relevant estimates over free and bound pieces.

Fix $N_{0}>0$ and choose $\varepsilon>0$ sufficiently small that properties (i)-(iv) enumerated at the start of the proof of Lemma 7.6 hold. We expand $\hat{\mu}_{\varepsilon}\left(\hat{H}_{\varepsilon}\right)$ precisely as in $(7.19)$. First, we must show that the second and third sums in that expression are the error terms in the expansion.

As in the proof of Lemma 7.10 , we call each $Y_{i}$ bound or free depending on whether $\hat{f}^{T_{i}}\left(Y_{i}\right)$ is undergoing a bound period at time $T_{i}$ or not. When summing over the free pieces, (7.29) implies that both sums are of order $\mathcal{O}\left(\hat{\mu}_{\varepsilon}\left(\hat{H}_{\varepsilon}\right) e^{-(\hat{\alpha}-\xi) N_{0}}\right)$ since the entry time for each such $Y_{i}$ to $\hat{H}_{\varepsilon}$ is greater than $N_{0}$. Similarly, we estimate the second and third sums in (7.19) over bound pieces $Y_{i}$ using the slow approach condition $(3.3)$ so that by $(7.31)$, these sums are $\mathcal{O}\left(\hat{\mu}_{\varepsilon}\left(\hat{H}_{\varepsilon}\right) e^{-\alpha N_{0} / 2}\right)$. We thus arrive at equation 7.20 as before.

Next, we derive (7.21) as before since that uses only property (iii) and the uniform log-Hölder property of the invariant density $g_{\varepsilon}$ (Lemma 7.1); so we obtain the same expressions with the same definition of $P_{\varepsilon}\left(S_{k} \hat{\varphi}\left(Z_{j}\right)\right)$.

Since the slow approach condition (3.3) implies that $z$ is disjoint from the post-critical orbit, we may choose $\varepsilon$ sufficiently small such that $f^{k}(c) \notin H_{\varepsilon}(z)$ for $k \leqslant p N_{0}$ and all $c \in$ Crit. Thus we may follow the proof of the simpler item (a) of Lemma 7.6, without having to consider the left and right 
halves of the hole separately. We use 7.25 to estimate the ratio in (7.21) and so arrive at (7.23) precisely as before.

Now, $\hat{\varphi}=-t \log |D f| \circ \hat{\pi}-P(-t \log |D f|)$. Although $\hat{\varphi}$ is not continuous on $\hat{I}_{z, \varepsilon_{0}, \varepsilon}$, it is still true on each $Y_{i}$ and for each orbit segment of length at most $p N_{0}$, that $S_{k} \hat{\varphi}$ is continuous with bounded ratio on $Y_{i}$ and each component of $\hat{H}_{\varepsilon}^{\prime}$ on level at most $p N_{0}$. This follows since we have trimmed

$L$-cylinders in our construction of $Y=\hat{I}^{\prime}(L)$. This extends to $Z_{j}$ since $\hat{f}^{-p N_{0}}(\hat{c}) \cap Z_{j}=\emptyset$ for each $Z_{j}$ by choice of $\varepsilon$, and so $P_{\varepsilon}\left(S_{k} \hat{\varphi}\left(Z_{j}\right)\right) \rightarrow 1$ as $\varepsilon \rightarrow 0$.

We thus arrive at 7.24 with error term $\mathcal{O}\left(N_{0} e^{-\tilde{\alpha} N_{0}}\right)$ and $\tilde{\alpha}=\min \{\hat{\alpha}-\xi, \alpha / 2\}$, and taking $N_{0} \rightarrow \infty$ completes the proof of the lemma.

Finally, Lemmas 7.10 and 7.11 together with Theorem 7.2 and Proposition 7.3 complete the proof of Theorem 3.7, using (7.3).

\section{REFERENCES}

[AB] V.S. Afraimovich and L.A. Bunimovich, Which hole is leaking the most: a topological approach to study open systems, Nonlinearity 23:3 (2010), 643-656.

[APT] E.G Altmann, J.S.E. Portela and T. Tél, Leaking chaotic systems, Rev. Mod. Phys. 85 (2013), 869-918.

[BV1] W. Bahsoun and S. Vaienti, Metastability of certain intermittent maps, Nonlinearity 25:1 (2012), $107-124$.

[BV2] W. Bahsoun and S. Vaienti, Escape rates formulae and metastability for randomly perturbed maps, Nonlinearity 26:5 (2013), 1415-1438.

[BLS] H. Bruin, S. Luzzatto and S. van Strien, Decay of correlations in one-dimensional dynamics, Ann. Scient. Éc. Norm. Sup. 36 (2003), 621-646.

[BDM] H. Bruin, M.F. Demers and I. Melbourne, Existence and convergence properties of physical measures for certain dynamical systems with holes, Ergod. Th. and Dynam. Sys. 30 (2010), 687-728.

[BDT] H. Bruin, M.F. Demers and M. Todd, Hitting and escaping statistics: mixing, targets and holes, Adv. Math. 328 (2018), 1263-1298.

[BRSS] H. Bruin, J. Rivera-Letelier, W. Shen, and S. van Strien, Large derivatives, backward contraction and invariant densities for interval maps, Invent. Math. 172 (2008), 509-533.

[BY] L.A Bunimovich and A. Yurchenko, Where to place a hole to achieve a maximal escape rate, Isr. J. Math. 182:1 (2011), 229-252.

[CM] N. Chernov and R. Markarian, Ergodic properties of Anosov maps with rectangular holes, Bol. Soc. Bras. Mat. 28 (1997), 271-314.

[CMT] N. Chernov, R. Markarian and S. Troubetzkoy, Conditionally invariant measures for Anosov maps with small holes, Ergod. Th. and Dynam. Sys. 18 (1998), 1049-1073.

[CMS] P. Collet, S. Martínez and B. Schmitt, The Yorke-Pianigiani measure and the asymptotic law on the limit Cantor set of expanding systems, Nonlinearity 7 (1994), 1437-1443.

[D1] M.F. Demers, Markov extensions and conditionally invariant measures for certain logistic maps with small holes, Ergod. Th. Dynam. Sys. 25 (2005), 1139-1171.

[D2] M.F. Demers, Dispersing billiards with small holes, in Ergodic Theory, Open Dynamics and Coherent Structures, Springer Proceedings in Mathematics 70 (2014), 137-170.

[DF] M.F. Demers and B. Fernandez, Escape rates and singular limiting distributions for intermittent maps with holes, Trans. Amer. Math. Soc. 368 (2016), 4907-4932.

[DIMMY] M.F. Demers, C. Ianzano, P. Mayer, P. Morfe and E. Yoo, Limiting distributions for countable state topological Markov chains with holes, Discrete and Contin. Dynam. Sys. 37:1 (2017), 105-130.

[DT1] M.F. Demers and M. Todd, Equilibrium states, pressure and escape for multimodal maps with holes, Isr. J. Math. 221:1 (2017), 367-424.

[DT2] M.F. Demers and M. Todd, Slow and fast escape for open intermittent maps, Comm. Math. Phys. 351:2 (2017), 775-835.

[DW] M.F. Demers and P. Wright, Behavior of the escape rate function in hyperbolic dynamical systems, Nonlinearity 25 (2012), 2133-2150.

[DWY] M.F. Demers, P. Wright and L.-S. Young, Escape rates and physically relevant measures for billiards with small holes, Commun. Math. Phys. 294 (2010), 353-388. 
[DG] C.P. Dettmann and O. Georgiou, Survival probability for the stadium billiard, Physica D 238 (2009), 23952403.

[DGKK] C.P. Dettmann, O. Georgiou, G. Knight and R. Klages, Dependence of chaotic diffusion on the size and position of holes, Chaos 22023132 (20012).

[DR] C.P. Dettmann and M.R. Rahman, Survival probability for open spherical billiards, Chaos 24043130 (2014).

[DoT] N. Dobbs and M. Todd, Free energy jumps up, Preprint (arXiv:1512.09245).

[DoW] D. Dolgopyat and P. Wright, The diffusion coefficient for piecewise expanding maps of the interval with metastable states, Stochastics and Dynamics 12 (2012), paper 1150005.

[FP] A. Ferguson, M. Pollicott, Escape rates for Gibbs measures, Ergodic Theory Dynam. Systems, 32 (2012), 961-988.

[FKMP] P.A. Ferrari, H. Kesten, S. Martínez and P. Picco, Existence of quasi-stationary distributions. A renewal dynamical approach, Annals of Prob. 23 (1995), 501-521.

[FFT1] A. C. M. Freitas, J. M. Freitas, and M. Todd, The compound Poisson limit ruling periodic extreme behaviour of non-uniformly hyperbolic dynamics. Comm. Math. Phys., 321 (2013), 483-527.

[FFT2] A. C. M. Freitas, J. M. Freitas, and M. Todd, Speed of convergence for laws of rare events and escape rates, Stochastic Process. Appl. 125 (2015), 1653-1687.

[FMS] G. Froyland, R. Murray and O. Stancevic, Spectral degeneracy and escape dynamics for intermittent maps with a hole, Nonlinearity 24 (2011), 2435-2463.

[GHW] C. Gonzalez-Tokman, B. Hunt and P. Wright, Approximating invariant densities for metastable systems, Ergod. Th. Dynam. Sys. 34 (2014), 1230-1272.

[H] F. Hofbauer, Piecewise invertible dynamical systems, Probab. Theory Relat. Fields 72 (1986), 359-386.

[HR] F. Hofbauer and P. Raith, Topologically transitive subsets of piecewise monotonic maps, which contain no periodic points. Monatsh. Math. 107 (1989), 217-239.

[IT1] G. Iommi and M. Todd, Natural equilibrium states for multimodal maps. Comm. Math. Phys., 300 (2010), 65-94.

[IT2] G. Iommi and M. Todd, Thermodynamic formalism for interval maps: inducing schemes. Dyn. Syst., 28 (2013), 354-380.

[K] G. Keller, Lifting measures to Markov extensions. Monatsh. Math. 108 (1989), 183-200.

[KL1] G. Keller and C. Liverani, Stability of the spectrum for transfer operators, Annali della Scuola Normale Superiore di Pisa, Classe di Scienze (4) Vol. XXVIII (1999), 141-152.

[KL2] G. Keller and C. Liverani, Rare events, escape rates and quasistationarity: some exact formulae, J. Stat. Phys. 135:3 (2009), 519-534.

[LR-L] H. Li and J. Rivera-Letelier, Equilibrium States of Weakly Hyperbolic One-Dimensional Maps for Hölder Potentials, Comm. Math. Phys., 328 (2014), 397-419.

[LM] C. Liverani and V. Maume-Deschamps, Lasota-Yorke maps with holes: conditionally invariant probability measures and invariant probability measures on the survivor set, Annales de l'Institut Henri Poincaré Probability and Statistics, 39 (2003), 385-412.

[MS] W. de Melo and S. van Strien. One-dimensional dynamics, Springer-Verlag: Berlin, 1993.

[PY] G. Pianigiani and J. Yorke, Expanding maps on sets which are almost invariant: decay and chaos, Trans. Amer.Math. Soc. 252 (1979), 351-366.

[PU] M. Pollicott and M. Urbanski, Open Conformal Systems and Perturbations of Transfer Operators, Lecture Notes in Mathematics 2206, Springer: Berlin, 2018.

[PR-L] F. Przytycki and J. Rivera-Letelier, Geometric pressure for multimodal maps of the interval, Mem. Amer. Math. Soc. 259 no. 1246.

[PR-LS] F. Przytycki, J. Rivera-Letelier and S. Smirnov, Equivalence and topological invariance of conditions for non-uniform hyperbolicity in the iteration of rational maps, Invent. Math. 151 (2003) 29-63.

[R-LS] J. Rivera-Letelier and W. Shen, Statistical properties of one-dimensional maps under weak hyperbolicity assumptions, Ann. Sci. Éc. Norm. Supér. (4), 47 (2014), 1027-1083.

[V] D. Vere-Jones, Geometric ergodicity in denumerable Markov chains, Quart. J. Math 13 (1962), 7-28.

[Y] L.S. Young, Some large deviation results for dynamical systems, Trans. Amer. Math. Soc. 318 (1990), $525-543$. 
Mark F. Demers, Department of Mathematics, Fairfield University, Fairfield, CT 06824, USA

Email address: mdemers@fairfield.edu

$U R L:$ http://faculty.fairfield.edu/mdemers

Mike Todd, Mathematical Institute, University of St Andrews, North Haugh, St Andrews, KY16 9SS, SCOTLAND

Email address: m.todd@st-andrews.ac.uk

$U R L:$ http://www.mcs.st-and.ac.uk/ miket/ 